\title{
Asymptotic analysis of the critical dynamics of spherical gaseous detonations.
}

\author{
Paul Clavin, Raúl Hernández Sánchez and Bruno Denet \\ Aix Marseille Université, CNRS, Centrale Marseille, IRPHE UMR7342, \\ 49 Rue F. Joliot Curie, 13384 Marseille, France \\ (Received ?; revised ?; accepted ?. - To be entered by editorial office )
}

The critical dynamics of supersonic combustion waves is studied in the context of the direct initiation of detonation in a spherical geometry. The study is performed by an asymptotic analysis in the limit of small heat release, including unsteadiness, curvature and the gradient of the burnt-gas flow. Derivation of analytical expressions for the rarefaction wave in the burnt-gas flow, combined with numerical studies, provides the basis of the analysis. The critical trajectories "detonation velocity versus front radius" $\mathcal{D}\left(r_{f}\right)$ are characterized by a decay well below the CJ velocity at a small radius (however larger than the detonation thickness), followed by a re-acceleration process back to a CJ detonation. The phenomenon is explained by the dynamics of the sonic point inside the inert rarefaction wave behind the reaction zone. The key mechanism is a critical slowdown as soon as the sonic condition (relative to the lead shock) approaches the reaction zone from behind, leading to an increase of the time delay in the nonlinear response of the combustion wave to the rarefaction-wave-induced decay. Detonation fails if the rate of decay is strong enough to prevent the sonic point to catch the reaction zone. Concerning successful initiation, the link between the trajectories $\mathcal{D}\left(r_{f}\right)$ of the fully unsteady problem and of the self-similar CJ solution of the discontinuous model is deciphered in the long time limit.

\section{Key words:}

\section{Introduction}

The dynamics of spherical gaseous detonations is controlled by small modifications of its inner structure which is thin compared to the radius. The direct initiation process is a typical example of such a sensitivity, see Clavin \& Denet (2020). For large activation energy, a critical radius larger than the detonation thickness by two orders of magnitude has been identified by He \& Clavin (1994) illustrating a quasi-steady curvature-induced quenching of spherical detonations. Direct numerical simulations have confirmed such a large critical radius but they also show that strong unsteadiness of the inner-detonationstructure is involved in the trajectories "propagation velocity versus radius", especially near criticality, see He \& Clavin (1994), He (1996), Eckett et al. (2000) and Ng \& Lee (2003). Investigating successful initiation far from the critical radius (supercritical regime) and neglecting the small gradient of the burnt-gas flow, Clavin \& Denet (2020) have shown that, near the CJ regime, unsteadiness is produced by the upstream-running compressible mode which controls the delayed response of the detonation structure to the burnt-gas flow. The analysis was performed in the limit of small heat release which provides us with a systematic framework for studying the problem which is one of two timescales. 
The present paper is an extension of the Clavin \& Denet (2020) analysis to the critical dynamics in a spherical geometry. The objective is to elucidate further the mechanism responsible for the complex dynamics observed near criticality in direct numerical simulations, namely re-ignition after a quasi-quenching of the detonation with a propagation velocity decreasing well below the CJ velocity accompanied by a substantial increase of the reaction-zone thickness, called sometimes "decoupling of the reaction from the lead shock". The problem is investigated here by an asymptotic analysis in the limit of small heat release, including unsteadiness, curvature and the gradient of the burnt-gas flow (rarefaction wave) at the exit of the reaction zone. The gradient of the rarefaction flow which is small at the scale of the detonation thickness, plays however an important role in the critical dynamics; it controls the dynamics of the sonic point inside the rarefaction wave behind the reaction wave. Our attention will be limited to stable or weakly unstable detonations.

In the limit of small heat release, the Mach number is everywhere close to unity, $0<M-1 \ll 1$, and the problem reduces to a single equation for the flow. In a preliminary step, an analytical solution is obtained for the rarefaction wave behind a detonation treated as a discontinuity. Near the detonation front, the solution presents the same local properties as in ordinary spherical detonations approaching the CJ regime. For example, the decrease-rate at the front is given by the curvature of the flow as in Liñan et al. (2012) in the limit of large Mach number. The analytical solution of the burnt gas flow is used as the external solution of the unsteady inner structure of curved detonations. The latter is analyzed with the same formalism as in Clavin \& Denet (2020). A combination of matched asymptotic method and numerical study of the asymptotic equation is then used for improving our understanding of the phenomenon. When the detonation velocity is larger than the planar CJ velocity the detonation is overdrive and the sonic point is outside the inner structure of the reaction wave. Near the critical threshold of direct initiation, the propagation velocity of the lead shock first decreases below the CJ velocity and the unsteady regime is still overdriven. The dynamics of the sonic point (sonic condition of the flow relative to the lead shock) then controls the subsequent critical dynamics that leads to either sustained detonation propagation or detonation failure. In that respect, the gradient of the burnt gas flow, even small, plays an important role, and analytical solutions for the rarefaction wave behind the reaction wave are quite useful.

The analysis also clarifies the question raised by Taylor (1950a) concerning the selfsimilar solution of the rarefaction flow behind a spherical CJ detonation, obtained by Zeldovich (1942) and Taylor (1950a) in the limit of large Mach number. The detonation being treated as a discontinuity, the radial rate of change of the flow becomes infinite on the detonation front. According to Taylor (1950a), it is unlikely that this result would be true if the modification of the inner structure is taken into account (non zero detonation thickness). To address this question, a self-similar solution is derived in the present article for the CJ detonation moving with a propagation Mach number larger than unity by a small amount $0<M-1 \ll 1$ (small heat release). Amazingly, the new selfsimilar solution is qualitatively similar to that obtained in the opposite limit $M \gg 1$; the gradient of the flow is also infinite on the front. However, this self-similar CJ flow is quite different from the unsteady flow behind an overdriven detonation approaching the CJ regime. The transitory flow, bridging the gap between the two flows, is obtained by a numerical solution of the asymptotic equation and is re-constructed by combining method of characteristics and analytical solutions. We will then see to what extent the self-similar flow is meaningful when the inner structure is taken into account.

The paper is organized as follows. The first part of the paper in sections 2 and 3 is devoted to the rarefaction wave in a spherical geometry in the limit $0<M-1 \ll 1$. The 
two-timescale nature of this flow is discussed in $\S 2.1$. The self-similar solution behind a spherical CJ detonation treated as a discontinuity is obtained in $\S 2.2$. The unsteady rarefaction wave behind an overdriven detonation is presented in $\S 2.3$. The transitory flow describing the transition from the overdriven regime to the self-similar CJ solution is obtained in section 3 for a detonation treated as a discontinuity. The rest of the paper is devoted to the study of the dynamics near criticality when the modifications to the inner structure is taken into account. The formulation of the problem and the method of solution are presented in $\S 4$.1. The asymptotic analysis of overdriven detonations near the CJ regime is performed in $\S 4.2$. The critical dynamics is discussed in section 5 where the numerical results are presented. Conclusions and perspectives are given in section 6 .

Five appendices are added. The jump conditions across a detonation treated as a discontinuity are recalled in $\S \mathrm{A}$. The linear acoustic wave in a spherical geometry is briefly recalled in $\S \mathrm{B}$ and compared with the nonlinear structure of the rarefaction wave for $0<M-1 \ll 1$. Technical details are presented in $\S \mathrm{C}$, including the calculation of the motion of the sonic point in the rarefaction wave. The decay of an inert spherical shock wave freely propagating in open space is presented in $\S \mathrm{D}$. The C-shaped curve of the steady-state approximation is briefly revisited in $\S \mathrm{E}$, extending the previous analysis to a non-uniform flow of burnt gas.

\section{Rarefaction wave behind a detonation treated as a discontinuity}

In principle, gaseous detonations could be treated as a discontinuity if the length scale of the burnt gas flow is larger than the detonation thickness, namely for a radius large enough in a spherical geometry. Even though the discontinuous model is not relevant for the critical dynamics, the unsteady solution of the rarefaction wave is first analyzed with this model in the limit of small heat release. This is a useful preliminary step providing us with the external solution of the solution including the modification of the inner structure.

\subsection{Two-time-scale analysis of the rarefaction wave}

In the limit of small heat release, the flow of burnt gas close to the detonation front is controlled by a single equation, which corresponds to equation (6.6) of Clavin \& Denet (2020) by setting the reaction rate equal to zero. In this section we show that this equation can be extended throughout the rarefaction wave. For that purpose we study the problem of fluid mechanics behind a supersonic discontinuity in the limit of a propagation velocity larger than the sound speed by a small amount. Denoting $\gamma, a, p$ and $u$ the ratio of specific heat, the isentropic speed of sound, the pressure and the gas velocity (relative to the laboratory frame where the uncompressed gas is at rest), an inert compressible flow in a spherical geometry is governed by two hyperbolic equations

$$
\frac{1}{\gamma p}\left[\frac{\partial}{\partial t}+(u \pm a) \frac{\partial}{\partial r}\right] p \pm \frac{1}{a}\left[\frac{\partial}{\partial t}+(u \pm a) \frac{\partial}{\partial r}\right] u=-2 \frac{u}{r}
$$

where $r$ is the radius and $t$ the time, see Liñan et al. (2012) and Clavin \& Denet (2020). For a detonation treated as a discontinuity, the pressure $p$ and the gas velocity $u$ on the detonation front $r=r_{f}(t)$ are given in terms of the propagation velocity $\mathcal{D}(t)$ by the conservation equations recalled in Appendix A, see (A 1)-(A 6),

$$
r=r_{f}(t): \quad \frac{1}{\gamma} \frac{p-p_{u}}{p_{u}}=M(t) \frac{u}{a_{u}}
$$


where $M(t) \equiv \mathcal{D}(t) / a$ is the Mach number and the subscript $\mathrm{u}$ identifies the constant properties upstream from the front. Written in the reference frame attached to the front equations (2.1) read

$$
\begin{gathered}
\mathrm{x} \equiv r-r_{f}(t) \leqslant 0, \quad \mathrm{~d} r_{f}(t) / \mathrm{d} t=\mathcal{D}(t) \\
\frac{1}{\gamma p}\left[\frac{\partial}{\partial t}+(u \pm a-\mathcal{D}) \frac{\partial}{\partial \mathrm{x}}\right] p \pm \frac{1}{a}\left[\frac{\partial}{\partial t}+(u \pm a-\mathcal{D}) \frac{\partial}{\partial \mathrm{x}}\right] u=-2 \frac{u}{r_{f}(t)+\mathrm{x}} .
\end{gathered}
$$

In the limit of small heat release $q_{m} / c_{p} T_{u} \equiv \epsilon^{2} \ll 1$, the velocity of the planar CJ wave in steady state is slightly higher than the sound speed, see (A 2),

$$
0<\left(\mathcal{D}_{o_{C J}}-a\right) / a \approx \epsilon \ll 1, \quad \mathcal{D}_{o_{C J}} \approx(1+\epsilon) a,
$$

and the attention is focused on detonations sufficiently close to the CJ regime

$$
0 \leqslant\left(\mathcal{D}-\mathcal{D}_{o_{C J}}\right) / \epsilon a=O(1) \quad \text { or smaller. }
$$

In the limit $\epsilon \ll 1,0<(M-1)=O(\epsilon)$, according to (A 1)-(A 6), the gas velocity is smaller than the speed of sound $u / a=O(\epsilon)$ at the front. The condition for which a weak shock wave $0<(M-1) \ll 1$ can be considered as a discontinuity is discussed in Clavin \& Williams (2002). According to (2.2), the relative pressure variation is as small as $u / a$ and the variation of $(1 / \gamma) \delta p / p-u / a$ is even smaller, of order $(M-1) u / a$, $(1 / \gamma) \delta p / p-u / a=O\left(\epsilon^{2}\right)$. The variation of the speed of sound is also of order $\epsilon^{2} \delta a / a=$ $O\left(\epsilon^{2}\right)$. Neglecting terms of order $\epsilon^{2}$, the variation of $a$ is negligible, and the isentropic relation $(1 / \gamma) \delta p / p \approx u / a$ holds at the front, up to the second order in the perturbation analysis for $\epsilon \ll 1$ under the conditions (2.4) and (2.5) for which the relative jump of entropy across the detonation front is of order $\epsilon^{2}$. Then, the rarefaction wave is a quasi-transonic flow which can be analyzed by a two-time-scale analysis.

It has been known long ago that the rarefaction wave $u(r, t)$ behind a spherical detonation is delimited by a weak discontinuity at the radius $r_{0}(t)$ of a core of stagnant gas $\left(r_{0}<r_{f}\right)$ which growths with the speed of sound

$$
\mathrm{d} r_{0}(t) / \mathrm{d} t=a, \quad r \leqslant r_{0}(t): u=0 \Rightarrow \mathrm{d} u\left(r_{0}(t), t\right) / \mathrm{d} t=0 ; \quad r>r_{0}(t): u>0 .
$$

The flow $u$ is oriented in the same direction as the propagation $\mathcal{D}>0, u \geqslant 0$, and is increasing monotonously from zero at $r=r_{0}(t)$ to a value (at the detonation front $r_{f}$ ) smaller than the speed of sound by a factor $\epsilon$, so that the ordering $u / a=O(\epsilon)$ holds throughout the rarefaction wave. The equations of the two characteristics $\mathcal{C}_{ \pm}$in $(2.3)$ involve two differential operators

$$
\partial / \partial t+V_{ \pm} \partial / \partial \mathrm{x}, \quad V_{ \pm} \equiv(u \pm a)-\mathcal{D} .
$$

The scalars $I_{+} \equiv(1 / \gamma) \delta p / p+u / a$ and $I_{-} \equiv(1 / \gamma) \delta p / p-u / a$ that are transported by $\mathcal{C}_{+}$ and $\mathcal{C}_{-}$respectively, are modified by the flow divergence on the right-hand side of $(2.3)$ whose order of magnitude is $\epsilon a / r$,

$$
\frac{\partial I_{ \pm}}{\partial t}+V_{ \pm} \frac{\partial I_{ \pm}}{\partial \mathrm{x}}=-2 \frac{u}{r}=O(\epsilon a / r)
$$

Under the conditions (2.4) and (2.5), the flow with respect to the front is quasi-transonic, $\mathcal{D}-u=O(\epsilon a)$. The propagation velocity $V_{-} \equiv u-a-\mathcal{D}<0$ of the downstream-running $\dagger$ characteristics $\mathcal{C}_{-}$is approximately $-a$ throughout the rarefaction wave. Near the detonation front where the flow relative to the front is subsonic $\mathcal{D}-u \leqslant a$ (overdriven regime),

$\dagger$ We use here the same convention as in Clavin \& Williams 2002; upstream-running (downstream-running) characterizes a propagation towards the shock (the core of stagnation gas) in the frame attached to the front. 
the characteristics $\mathcal{C}_{+}$is upstream-running $V_{+}=a-(\mathcal{D}-u)>0$. But a sonic point appears when $u$ decreases since $V_{+}=0$ when $\mathcal{D}-u=a$. Therefore the characteristics $\mathcal{C}_{+}$ becomes downstream-running $\left(V_{+}<0\right)$ behind the sonic point which stands close to the front because we consider propagation regimes of the detonation that are close to the CJ regime which is characterized by the sonic condition at the detonation front. Then the transport of $I_{-}$by $\mathcal{C}_{-}$from the sonic point to $r_{0}$ is quasi-instantaneous compared to the slow transport of $I_{+}$by $\mathcal{C}_{+},\left|V_{+}\right| / a=O(\epsilon),\left|V_{-}\right| / a \approx 1$. Anticipating that the thickness of the rarefaction wave $\Delta r \equiv\left(r_{f}-r_{0}\right)$ is smaller than the detonation radius $\Delta r / r_{f}=O(\epsilon)$, see $\S 2.2$, the modification of $I_{-}$by the geometrical effect during the transit time $\Delta r / a$ of $\mathcal{C}_{-}$is of order $\epsilon \Delta r / r_{f}=O\left(\epsilon^{2}\right)$ and can be neglected. Then, to leading order in the limit $\epsilon \ll 1$, the isentropic relation of acoustics $(1 / \gamma) \delta p / p=u / a$, which is valid inside the detonation structure in the limit of small heat release, holds throughout the rarefaction wave $u \ll a, u / \epsilon a=O(1)$. Then, neglecting the short time delay and the small flow modification (of order $\epsilon^{2} a$ ) introduced by the fastest downstream-running mode and retaining only the slow timescale, the leading order of the flow $u(\mathrm{r}, t)$ is controlled by a single equation corresponding to the simple wave associated with $\mathcal{C}_{+}$,

$$
\begin{gathered}
{\left[\frac{\partial}{\partial t}+(u+a) \frac{\partial}{\partial r}\right] u=-a \frac{u}{r}} \\
\mathrm{x} \equiv r-r_{f}(t) \leqslant 0,\left[\frac{\partial}{\partial t}+(u+a-\mathcal{D}) \frac{\partial}{\partial \mathrm{x}}\right] u=-a \frac{u}{r_{f}(t)+\mathrm{x}}, \quad \frac{\mathrm{d} r_{f}}{\mathrm{~d} t}=\mathcal{D}(t) .
\end{gathered}
$$

\subsection{Self-similar rarefaction wave behind a spherical CJ detonation}

Generally speaking when the modification to the inner structure of the leading front is ignored (zero detonation-thickness), a self-similar solution exists when a finite amount of energy is deposited quasi-instantaneously by a quasi-punctual external source at the centre because there is no length and time scales in the problem. This is the case for the blast wave of Sedov (1946) and Taylor (1950b) and the rarefaction wave of Zeldovich (1942) and Taylor (1950a) behind a CJ detonation, obtained in the limit of large Mach number $M \gg 1$, see textbooks, for example Clavin \& Searby (2016). We show below that, in the opposite limit of small heat release $M_{O_{C J}}-1 \ll 1$, there is also a self-similar solution for the rarefaction wave behind a CJ detonation, which is qualitatively similar to the self-similar solution of Zeldovich (1942) and Taylor (1950a). The difference concerns mainly the extension of the self-similar rarefaction wave which is small compared to the radius of the detonation in the limit $M_{o_{C J}}-1 \ll 1$.

\subsubsection{Formulation}

Consider the spherical detonation propagating with the constant CJ velocity $\mathcal{D}_{o_{C J}} \approx$ $(1+\epsilon) a$ in the limit $(2.4) \epsilon \ll 1$. According to the conservation equations (A 1)-(A 6), the boundary condition at the front takes the form

$$
\mathrm{x}=0: \quad u \approx \epsilon a .
$$

After dividing by $\epsilon a$ and introducing the non dimensional flow of order unity in the limit $\epsilon \ll 1, \mathrm{v}(\mathrm{x}, t) \equiv u /(\epsilon a), \mathrm{v} \in[0,1]$, equation (2.10) yields

$$
\begin{aligned}
{\left[\frac{\partial}{\partial t}+\left(u+a-\mathcal{D}_{o_{C J}}\right) \frac{\partial}{\partial \mathrm{x}}\right] \mathrm{v} } & =-a \frac{\mathrm{v}}{r_{f}(t)+\mathrm{x}}, \quad \mathrm{v} \equiv \frac{u}{\epsilon a}, \\
{\left[\frac{1}{a} \frac{\partial}{\partial t}+(\mathrm{v}-1) \epsilon \frac{\partial}{\partial \mathrm{x}}\right] \mathrm{v} } & =-\frac{\mathrm{v}}{r_{f}(t)+\mathrm{x}}
\end{aligned}
$$


where (2.4) has been used. This suggests to rescale the distance from the front by using the new space variable $\eta \equiv \mathrm{x} / \epsilon$, so that equations (2.11) and (2.13) read

$$
\eta \equiv\left[r-r_{f}(t)\right] / \epsilon, \quad\left[\frac{1}{a} \frac{\partial}{\partial t}+(\mathrm{v}-1) \frac{\partial}{\partial \eta}\right] \mathrm{v}=-\frac{\mathrm{v}}{r_{f}(t)+\epsilon \eta} ; \quad \eta=0: \mathrm{v}=1 .
$$

The downstream relation (2.6) is automatically fulfilled at $\eta=\eta_{0}(t) \equiv\left[r_{0}(t)-r_{f}(t)\right] / \epsilon$ since the relations $\mathrm{v}\left(\eta_{0}(t), t\right)=0$ and $\mathrm{dv}\left(\eta_{0}(t), t\right) / \mathrm{d} t=0$ are verified by $(2.14), \mathrm{v}=0$ : $\partial \mathrm{v} / \partial t-a \partial \mathrm{v} / \partial \eta=0$, yielding $\mathrm{d} \eta_{0}(t) / \mathrm{d} t=-a$, so that, using $(2.4) \mathrm{d} r_{f} / \mathrm{d} t=(1+\epsilon) a$, one gets $\mathrm{d} r_{0} / \mathrm{d} t=a$.

Introducing the two lengths $r_{f i}$ and $r_{0 i}$ characterizing the initial condition $t=0$,

$$
\begin{aligned}
r_{f}(t) & =\mathcal{D}_{o_{C J}} t+r_{f i} \approx(1+\epsilon) a t+r_{f i}, \quad r_{0}(t)=a t+r_{0 i}, \quad r_{f i}>r_{0 i}, \\
\eta_{0}(t) & \equiv-\left[r_{f}(t)-r_{0}(t)\right] / \epsilon=-a t+\eta_{0 i}, \quad \eta_{0 i} \equiv-\left(r_{f i}-r_{0 i}\right) / \epsilon<0
\end{aligned}
$$

$\epsilon\left|\eta_{0 i}\right|$ being the initial thickness of the rarefaction wave, we will show below that a selfsimilar solution of (2.14) exists in the limit $\epsilon \ll 1$ if the initial thickness of the rarefaction wave $r_{f i}-r_{0 i}$ is smaller than the initial radius of the detonation $r_{f i}$ by a factor $\epsilon$, as it is the case in the linear solution presented in Appendix B.1,

$$
\left(r_{f i}-r_{0 i}\right) / r_{0 i}=O(\epsilon), \quad r_{f}(t)-r_{0}(t)=\epsilon\left(a t-\eta_{0 i}\right), \quad\left|\eta_{0 i}\right|=O\left(r_{0 i}\right) .
$$

To leading order, the denominator $r=r_{f}+\epsilon \eta$ on the right-hand side of (2.14) can be replaced by $r_{0}(t)$ throughout the rarefaction wave $r_{0}(t) \leqslant r \leqslant r_{f}(t)$ since, according to $(2.17), r_{f}(t)=r_{0}(t)+\epsilon a t-\epsilon \eta_{0 i}$ so that $r_{f}(t)=(1+\epsilon) r_{0}(t)+O\left(\epsilon r_{0 i}\right)$. Therefore, replacing the time variable $t$ by a time-like variable $\nu \equiv r_{0}(t)=a t+r_{0 i}$ whose dimension is a length, equation (2.14) for the flow $\mathrm{v}(\eta, \nu)$ takes the form of a Burgers-like equation, free from parameter, in which the local viscous dissipation is replaced by a global (linear) damping rate on the right-hand side,

$$
\begin{aligned}
& \epsilon \rightarrow 0: \quad\left[\frac{\partial}{\partial \nu}+(\mathrm{v}-1) \frac{\partial}{\partial \eta}\right] \mathrm{v}=-\frac{\mathrm{v}}{\nu}, \quad \eta=0: \quad \mathrm{v}=1 \\
& \text { where } \quad \nu \equiv a t+r_{0 i}, \quad \eta \equiv \frac{r-r_{f}(t)}{\epsilon}, \quad \mathrm{v}(\eta, \nu) \equiv \frac{u(\mathrm{x}, t)}{\epsilon a} .
\end{aligned}
$$

The parameter $r_{0 i}$ in the definition of the time-like variable $\nu$ can be eliminated by a change of time origin.

\subsubsection{Infinite gradient of the flow on the front. Self-similar solution}

As in the self-similar solution of Zeldovich (1942) and Taylor (1950a) obtained in the opposite limit of large Mach number $\mathcal{D}_{o_{C J}} / a \gg 1$, the solution of $(2.18)$ is singular on the detonation front where the gradient of the flow becomes infinite. The flow being constant on the front $\mathrm{v}=1$, the unsteady term $\partial \mathrm{v} / \partial \nu$ in (2.18) is negligible around $\eta=0^{-}$so that the steady-state approximation holds near the detonation front $(\mathrm{v}-1) \partial \mathrm{v} / \partial \eta \approx-1 / \nu$

$$
1-\mathrm{v}=\sqrt{\frac{2}{\nu}(-\eta)}, \quad 1-\frac{u}{\epsilon a}=\sqrt{\frac{2\left[r_{f}(t)-r\right]}{\epsilon\left(a t+r_{0 i}\right)}},
$$

the time derivative $\partial \mathrm{v} /\left.\partial \nu\right|_{\eta=0^{-}}=\sqrt{-2 \eta} /\left(2 \nu^{3 / 2}\right)$ being negligible in a boundary layer at the front $|\partial \mathrm{v} / \partial \nu|_{\eta=0^{-}} \ll 1 / \nu$,

$$
|\eta| \ll 2 \nu, \quad r_{f}(t)-r \ll 2 \epsilon\left(a t+r_{0 i}\right) .
$$

The divergence of the flow gradient on the front of a spherical CJ detonation $\partial \mathrm{v} /\left.\partial \eta\right|_{\eta=0^{-}} \propto$ $1 /(-\eta)^{1 / 2}$ is a consequence of the sonic condition $\eta=0: \mathrm{v}-1=0 \forall t$. 


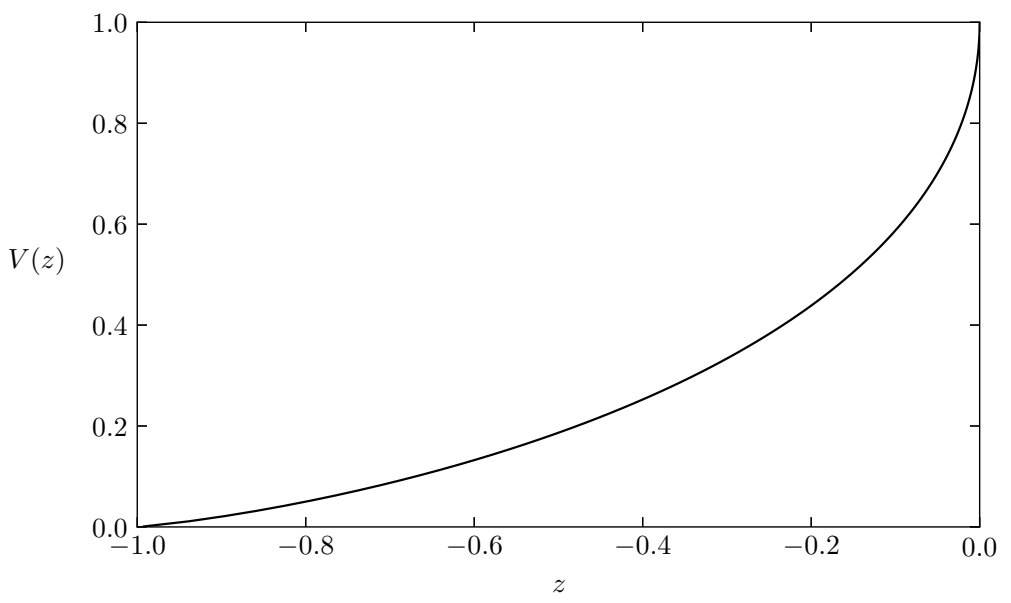

Figure 1. Solution of (2.23)-(2.24) representing the self-similar rarefaction flow behind the front of a spherical CJ detonation considered as a discontinuity for small heat release $\left(\mathcal{D}_{o_{C J}}-a\right) / a \approx \epsilon \ll 1$. The reduced flow $\mathrm{v}=\mathrm{u}(r, t) / \epsilon a$ is plotted versus the reduced distance from the front $z=\left(r-r_{f}(t)\right) / \epsilon r_{0}(t)$ with $r_{f}(t)=\mathcal{D}_{o_{C I}} t+r_{f i}, r_{0}(t)=a t+r_{0 i}$ and, according to $(2.26), r_{f i}=(1+\epsilon) r_{0 i}$.

The self-similar solution of (2.18) is obtained by looking for a solution in the form

$$
\begin{gathered}
\mathrm{v}(\eta, \nu)=\mathrm{V}(z) \quad \text { with } \quad z \equiv \eta / \nu \quad \Rightarrow \quad \frac{u}{\epsilon a}=V\left(\frac{\left.r-r_{f}(t)\right)}{\epsilon r_{0}(t)}\right) \\
{[-(1+z)+V] \frac{\mathrm{d} V}{d z}=-V, \quad z=0: \quad V=1 .}
\end{gathered}
$$

After multiplication by $1 / V^{2}$, equation $(2.23)$ takes the form $\mathrm{d}[(1+z) / V] / \mathrm{d} z+(1 / V) \mathrm{d} V / \mathrm{d} z=$ 0 , then, the solution $V(z)$ satisfying the boundary condition at $z=0$ is the root of a transcendental equation

$$
V \ln V-V+(z+1)=0 .
$$

According to this equation, the radius $r=r_{0}(t)$ of the spherical core of stagnant gas $V=0$ corresponds to $z=-1$. Therefore, $r_{0}(t)$ and $r_{f}(t)$ are linked by the relation

$$
z \equiv\left[r_{0}(t)-r_{f}(t)\right] / \epsilon r_{0}(t)=-1 \quad \Leftrightarrow \quad(1+\epsilon) r_{0}(t)=r_{f}(t)
$$

in agreement with the assumption (2.17) in the limit of small heat release (2.4), which finally takes the more restrictive form

$$
\left(r_{f i}-r_{0 i}\right)=\epsilon r_{0 i},
$$

in a consistent way with $r_{f}(t) \approx(1+\epsilon) a t+r_{f i}$ and $r_{0}(t)=a t+r_{0 i}$ yielding $(2.26)$ for $z=$ -1 . The velocity profile of the self-similar rarefaction wave is plotted in Figure 1 . Close to the detonation front $z \approx 0$, namely for $V=1+\delta V$ with $|\delta V| \ll 1, \ln V \approx \delta V-\delta V^{2} / 2+$.., $V \ln V \approx-1+(\delta V)^{2} / 2+$. so that equation $(2.24)$ yields $(\delta V)^{2} / 2+z \approx 0$ and the relation (2.20) is recovered for $|z| \ll 1, V \approx 1-\sqrt{-2 z}$. At the radius of the stagnant core, the root of (2.24) goes to zero $\lim _{z=-1} V=0^{+}$with a zero gradient $\mathrm{d} V /\left.\mathrm{d} z\right|_{z=-1}=0$, as shown by taking the limit $V \rightarrow 0^{+}$of the derivative of $(2.24) \mathrm{d}(V \ln V) / \mathrm{d} z-\mathrm{d} V / \mathrm{d} z+1=0$ leading to $\lim _{V \rightarrow 0^{+}}(\mathrm{d} V / \mathrm{d} z)=-2 / \ln V \rightarrow 0^{+}$.

To conclude the rarefaction wave behind a spherical CJ detonation sustained by a small 
heat release $\left(M_{o_{C J}}-1 \approx \epsilon \ll 1\right)$ is similar to the self-similar solution for $M_{o_{C J}} \gg 1$. The only difference is quantitative; the extension of the rarefaction wave is smaller than the detonation radius $r_{f}(t)$ by a factor $M_{o_{C J}}-1,\left(r_{f}-r_{0}\right) / r_{f} \approx\left(M_{o_{C J}}-1\right) / M_{o_{C J}}$ for small heat release while $\left(r_{f}-r_{0}\right) / r_{f}=1 / 2$ for $M_{o_{C J}} \gg 1$.

\subsection{Rarefaction wave behind an overdriven detonation considered as a discontinuity}

Within the framework of the the discontinuous model the decay of the propagation velocity of an overdriven detonation $\mathcal{D}(\tau)$ in a spherical geometry to the planar ChapmannJouguet velocity $\mathcal{D}_{o_{C J}}$ occurs systematically after a finite time and at a finite radius. In this section, we derive an analytical expression for the rarefaction wave behind an overdriven detonation treated as a discontinuity approaching the CJ velocity, in the limit $\epsilon \ll 1$. The subsequent relaxation to the self-similar solution is discussed in section 3 .

\subsubsection{Formulation}

As already mentioned, there is no length (or time) scale in the direct-initiation problem with the discontinuous model. However, in view of bridging the gap with the study in the second part of this manuscript, it is useful to introduce the non-dimensional space and time variables $\xi$ and $\tau$ as well as the reduced flow field $\mu(\xi, \tau)$ that are of order unity inside the unsteady (and curved) inner structure of the detonation in the limit (2.4)-(2.5)

$$
\begin{aligned}
\xi & \equiv \frac{\left(r-r_{f}(t)\right)}{l}, \quad \tau \equiv \epsilon \frac{a t}{l}, \quad \mathcal{D}=\frac{\mathrm{d} r_{f}}{\mathrm{~d} t}, \\
\mu(\xi, \tau) & \equiv \mathrm{v}-1=u / \epsilon a-1 \Leftrightarrow u / a=\epsilon(1+\mu)
\end{aligned}
$$

where $l$ is the detonation thickness and $1 / t_{r}=a / l$ the reaction rate at the Neumann state,

$$
\frac{\partial}{\partial r}=\frac{1}{l} \frac{\partial}{\partial \xi}, \quad \frac{\partial}{\partial t}=\frac{\epsilon}{t_{r}} \frac{\partial}{\partial \tau}-\frac{\mathcal{D}}{l} \frac{\partial}{\partial \xi} .
$$

For clarity, the results will be written with the two types of variables. Introducing the reduced radius $\tilde{r}_{f}(\tau)$ and propagation velocity $\dot{\alpha}_{\tau}(\tau)$

$$
\tilde{r}_{f}(\tau) \equiv \epsilon r_{f}(\tau) / l, \quad \dot{\alpha}_{\tau}(\tau) \equiv\left[\mathcal{D}(\tau)-\mathcal{D}_{o_{C J}}\right] /(\epsilon a),
$$

equation (2.10) reduces to (6.6) in Clavin \& Denet (2020) without the reaction term

$$
\begin{gathered}
\frac{\partial \mu}{\partial \tau}+\left(\mu-\dot{\alpha}_{\tau}\right) \frac{\partial \mu}{\partial \xi}=-\frac{1}{\tilde{r}_{f}(\tau)+\epsilon \xi}(1+\mu) \\
\text { where } \frac{\mathrm{d} \tilde{r}_{f}(\tau)}{\mathrm{d} \tau}=\frac{\mathcal{D}}{a}=(1+\epsilon)+\epsilon \dot{\alpha}_{\tau}(\tau), \quad \tilde{r}_{f}(\tau)=\tilde{r}_{f i}+(1+\epsilon) \tau+\epsilon \int_{0}^{\tau} \dot{\alpha}_{\tau} \mathrm{d} \tau
\end{gathered}
$$

see Appendix C.1. In (2.31), $\tilde{r}_{f i} \equiv \epsilon r_{f i} / l$ is associated with the initial position of the front $r_{f i} \equiv r_{f}(0)$. The sonic condition with respect to the front $(\mathcal{D}-u)=a$ corresponds to $\left(\mu-\dot{\alpha}_{\tau}\right)=0$, and a subsonic condition $(\mathcal{D}-u)<a$ corresponds to $\left(\mu-\dot{\alpha}_{\tau}\right)>0$ while $\left(\mu-\dot{\alpha}_{\tau}\right)<0$ characterizes a supersonic flow relative to the detonation front. The decay of the detonation velocity to the CJ velocity corresponds to $\dot{\alpha}_{\tau} \rightarrow 0^{+}$and $\mu_{f} \rightarrow 0^{+}$.

As already mentioned, the radius $r=r_{0}(\tau)$ of the spherical core of stagnant gas $u\left(r_{0}(\tau), \tau\right)=0$

$$
r<r_{0}(\tau): u=0, \quad r>r_{0}(\tau): u>0, \quad \partial u /\left.\partial r\right|_{r=r_{0}^{+}} \geqslant 0
$$

is a weak discontinuity of the flow moving at the speed of sound $\mathrm{d} r_{0}(t) / \mathrm{d} t=a$. Intro- 
ducing the reduced thickness of the rarefaction wave $\left|\xi_{0}(\tau)\right|$

$$
\xi_{0}(\tau) \equiv \frac{\left[r_{0}(\tau)-r_{f}(\tau)\right]}{l}<0 ; \quad \xi=\xi_{0}(\tau): \mu=-1 \quad \text { i.e. } \quad \mu\left(\xi_{0}(\tau), \tau\right)=-1,
$$

the weak discontinuity is recovered in $(2.31)$ at $\xi=\xi_{0}(\tau)$ where $\partial \mu /\left.\partial \tau\right|_{\xi=\xi_{0}^{+}}-(1+$ $\left.\dot{\alpha}_{\tau}\right) \partial \mu /\left.\partial \xi\right|_{\xi=\xi_{0}^{+}}=0$ and $\mu=-1$ by differentiating the last expression in $(2.33), \mathrm{d} \mu\left(\xi_{0}(\tau), \tau\right) / \mathrm{d} \tau=$ $\partial \mu /\left.\partial \tau\right|_{\xi=\xi_{0}^{+}}+\left(\mathrm{d} \xi_{0} / \mathrm{d} \tau\right) \partial \mu /\left.\partial \xi\right|_{\xi=\xi_{0}^{+}}=0$ yielding

$$
\mathrm{d} \xi_{0} / \mathrm{d} \tau=-\left(1+\dot{\alpha}_{\tau}\right), \quad \xi_{0}(\tau)=-\tau-\int_{0}^{\tau} \dot{\alpha}_{\tau}\left(\tau^{\prime}\right) \mathrm{d} \tau^{\prime}+\xi_{0 i}
$$

which corresponds effectively to $\mathrm{d} r_{0} / \mathrm{d} t=a$ that is $\mathrm{d}\left(r_{0}-r_{f}\right) / \mathrm{d} t=a-\mathcal{D}$ with, according to $(2.4)$ and $(2.30),(a-\mathcal{D})=-\epsilon a\left(1+\dot{\alpha}_{\tau}\right)$. When the order of magnitude of $\dot{\alpha}_{\tau}$ is not larger than unity in the limit $\epsilon \rightarrow 0,(\mathcal{D}-a) / a=O(\epsilon)$, equation (2.34) shows that the thickness of the rarefaction wave $r_{f}(t)-r_{0}(t)$ increases with a velocity of order $\epsilon a$ smaller than the speed of sound by a factor $\epsilon$. Considering $\tau=O(1)$ in the limit of small heat release, $\lim _{\epsilon \rightarrow 0} \epsilon \xi_{0}=0$, equation (2.32) yields $\lim _{\epsilon \rightarrow 0} \tilde{r}_{f}=\tau+\tilde{r}_{f i}$, so that, to leading order, equation (2.31) reduces to

$$
\epsilon \rightarrow 0, \quad \frac{\partial \mu}{\partial \tau}+\left[\mu-\dot{\alpha}_{\tau}(\tau)\right] \frac{\partial \mu}{\partial \xi}=-\frac{1+\mu}{\tau+\tilde{r}_{f i}} \quad \text { where } \quad \tilde{r}_{f i} \equiv \epsilon \frac{r_{f i}}{l}=O(1)
$$

Rescaling the non-dimensional length $\xi$ and time $\tau$ with $\tilde{r}_{f i} \equiv \epsilon r_{f i} / l$,

$$
\tilde{\tau} \equiv \frac{\tau}{\tilde{r}_{f i}}=\frac{a t}{r_{f i}}, \quad \tilde{\xi} \equiv \frac{\xi}{\tilde{r}_{f i}}=\frac{r-r_{f}(t)}{\epsilon r_{f i}}, \quad \tilde{\xi}_{0}(\tilde{\tau}) \equiv \frac{\xi_{0}(\tau)}{\tilde{r}_{f i}}=\frac{r_{0}(t)-r_{f}(t)}{\epsilon r_{f i}}
$$

equations (2.34)-(2.35) take a form free from parameter

$$
\frac{\partial \mu}{\partial \tilde{\tau}}+\left[\mu-\dot{\alpha}_{\tau}(\tilde{\tau})\right] \frac{\partial \mu}{\partial \tilde{\xi}}=-\frac{(1+\mu)}{\tilde{\tau}+1}, \quad \frac{\mathrm{d} \tilde{\xi}_{0}}{\mathrm{~d} \tilde{\tau}}=-\left[1+\dot{\alpha}_{\tau}(\tilde{\tau})\right]
$$

Once a general solution of (2.37) is known, the dynamics of the front $\dot{\alpha}_{\tau}(\tilde{\tau})$ is obtained by a boundary condition at the front. For the discontinuous model, the instantaneous flow of burned gas at the front, denoted by $\mu_{f}(\tilde{\tau}) \equiv \mu(\tilde{\xi}=0, \tilde{\tau})$, is given by the conservation of mass, momentum and energy in appendix A, leading to express $\mu_{f}(\tilde{\tau})$ in terms of $\dot{\alpha}_{\tau}(\tilde{\tau})$

$$
\tilde{\xi}=0: \quad \mu \equiv \mu_{f}(\tilde{\tau})=\mathcal{M}_{f}\left(\dot{\alpha}_{\tau}(\tilde{\tau})\right) .
$$

Equations (2.37) and (2.38) represent an eigenvalue problem in which the unknown function $\dot{\alpha}_{\tau}(\tilde{\tau})$ appears in the boundary condition (2.38) and in equation (2.37).

\subsubsection{Simplified formulation near the CJ velocity}

The formulation gets simpler when the attention is focused on the end of the detonation decay when the velocity is close to the CJ velocity,

$$
0<\frac{\left(\mathcal{D}_{i}-\mathcal{D}_{o_{C J}}\right)}{\epsilon a} \ll 1, \quad 0 \leqslant \dot{\alpha}_{\tau} \equiv \frac{\left(\mathcal{D}-\mathcal{D}_{o_{C J}}\right)}{\epsilon a} \ll 1,
$$

Expanding (A 1)-(A 2) for small values of $\left(\mathcal{D}-\mathcal{D}_{o_{C J}}\right) / \epsilon a$ yields the well known square root relation between the flow of burnt gas at the front $u_{b}(t)$ and the detonation velocity $\mathcal{D}(\tau)$ near the CJ regime $u_{b} / \epsilon a \approx 1+\sqrt{2\left(\mathcal{D}-\mathcal{D}_{o_{C J}}\right) / \epsilon a}$, obtained by the relation $\left(M_{o_{C J}}-M_{o_{C J}}^{-1}\right)^{2} /\left(M-M^{-1}\right)^{2} \approx 1-2\left(\mathcal{D}-\mathcal{D}_{o_{C J}}\right) / \epsilon a$ in (A 1). Then the boundary con- 
dition at the front simplifies to

$$
\begin{gathered}
u_{f}(\tau) / \epsilon a \approx 1+\sqrt{2\left(\mathcal{D}(\tau)-\mathcal{D}_{o_{C J}}\right) / \epsilon a}, \quad \mu_{f}(\tau) \approx \sqrt{2 \dot{\alpha}_{\tau}(\tau)} \ll 1 \\
0<\mu_{f i} \equiv u_{f i} / \epsilon a-1 \approx \sqrt{2\left(\mathcal{D}_{i}-\mathcal{D}_{o_{C J}}\right) / \epsilon a} \ll 1,
\end{gathered}
$$

where $u_{f i}$ and $\mu_{f i}$ denote the initial value of $u_{f}(\tau)$ and $\mu_{f}(\tau) \equiv u_{f i} / \epsilon a-1$ with $u_{f i}>\epsilon a$. The term $\left[\mu-\dot{\alpha}_{\tau}(\tilde{\tau})\right]$ can then be replaced by $\mu$ in $(2.35)$ because the flow field in the rarefaction wave increases monotonously with the radius, from $u=0$ at $r=r_{0}$ to a positive value $u_{f}$ on the front $\left(r=r_{f}\right), \mu \in\left[-1, \mu_{f}\right]$, with, according to (2.39)-(2.40), $0 \leqslant \dot{\alpha}_{\tau} \ll \mu_{f} \ll 1$ so that $\dot{\alpha}_{\tau}(\tau) \ll|\mu(\xi, \tau)| \forall \xi \in\left[\xi_{0}, 0\right]$. Under the condition (2.39), the unknown velocity of the front $\dot{\alpha}_{\tau}(\tilde{\tau})$ does not appear explicitly anymore on the left-hand side of equation (2.37) which reduces to the Burgers-like equation in (2.18),

$$
\epsilon \rightarrow 0, \quad 0<\dot{\alpha}_{\tau} \ll 1 \Rightarrow \frac{\partial \mu}{\partial \tilde{\tau}}+\mu \frac{\partial \mu}{\partial \tilde{\xi}}=-\frac{(1+\mu)}{\tilde{\tau}+1}, \quad \tilde{\xi}_{0}(\tilde{\tau})=-\tilde{\tau}+\tilde{\xi}_{0 i},
$$

the unknown function being the flow at the front $\mu_{f}(\tilde{\tau}) \equiv u_{f}(\tilde{\tau}) / \epsilon a-1$.

As we shall see in $\S 3.1$, the solution $\mu(\tilde{\xi}, \tilde{\tau})$ of $(2.42)$ reaches the self-similar CJ solution (2.23)-(2.24) in the long time limit if the initial thickness of the rarefaction wave scales as (2.17), $\left|\tilde{\xi}_{0 i}\right|=O(1)$ in the limit $\epsilon \rightarrow 0$. Notice that equation $(2.42)$, divided by $r_{f i}$, yields (2.18) for $\mathrm{v}=\mu+1$, the difference with the CJ problem being the boundary condition on the front $(\tilde{\xi}=0)$ which now involves an unknown flow $\mu_{f}(\tilde{\tau}) \neq 0, \mathrm{v}(\eta=0, \nu) \neq 1$.

\subsubsection{Analytical solutions.}

The rarefaction wave behind a spherical detonation is a nonlinear solution of the Euler equations, which cannot be described by a linearized approximation, even if the flow velocity is smaller than the speed of sound as it is the case in the limit of small heat release. By comparison, the linear solution is briefly recalled in Appendix B. Equations (2.37) and (2.42) have analytical solutions $\mu(\tilde{\xi}, \tilde{\tau})$ which provide us with an expression of the unsteady flow on the front $\mu_{f}(\tilde{\tau})=\mu(0, \tilde{\tau})$ in terms of the unknown function $\dot{\alpha}_{\tau}(\tilde{\tau})$ which, according to $(2.30)$, represents the propagation velocity of the front $\mathcal{D}(t)$. The dynamics of the front $\dot{\alpha}_{\tau}(\tilde{\tau})$ is then obtained in a second step through the boundary condition on the front. For example, the Rankine-Hugoniot condition yields the relaxation of a pure shock freely propagating in a spherical geometry which is derived in Appendix D. The end of the decay of an overdriven detonation, treated as a discontinuity, is obtained from (2.42) by using the boundary condition (2.40).

Analytical solutions of (2.37) are obtained in the form of separated variables,

$$
\mu(\tilde{\xi}, \tilde{\tau})=A(\tilde{\tau}) B(\tilde{\eta})-1, \quad \tilde{\eta} \equiv \tilde{\xi}-\tilde{\xi}_{0}(\tilde{\tau}), \quad \mu\left(\tilde{\xi}_{0}(\tilde{\tau}), \tau\right)=-1 \quad \frac{\mathrm{d} \tilde{\xi}_{0}}{\mathrm{~d} \tilde{\tau}}=-\left(1+\dot{\alpha}_{\tau}\right)
$$

Introducing the notation $B^{\prime} \equiv \mathrm{d} B / \mathrm{d} \tilde{\eta}$ and $\dot{A} \equiv \mathrm{d} A / \mathrm{d} \tilde{\tau}, \partial \mu / \partial \tilde{\tau}=\dot{A}+\left(1+\dot{\alpha}_{\tau}\right) A B^{\prime}$, $\mu \partial \mu / \partial \tilde{\xi}=\left(A B-\dot{\alpha}_{\tau}\right) A B^{\prime}$, the unknown function $\dot{\alpha}(\tilde{\tau})$ disappears from the equations for $A(\tilde{\tau})$ and $B(\tilde{\eta})$, yielding

$$
\dot{A} B+A^{2} B B^{\prime}=-A B /(\tilde{\tau}+1) \quad \Rightarrow \quad-\mathrm{d} A^{-1} / \mathrm{d} \tilde{\tau}+A^{-1} /(\tilde{\tau}+1)=-B^{\prime},
$$

the second equation being obtained after division by $A^{2} B$. The left-hand side of the second equation in (2.44) is a function of $\tilde{\tau}$ only, while the right-hand side is a function of $\tilde{\eta}$. Therefore the two sides should be equal to the same constant yielding an ordinary differential equation for $A(\tilde{\tau})$ and $B(\tilde{\eta})$ respectively

$$
\mathrm{d} A^{-1} / \mathrm{d} \tilde{\tau}-A^{-1} /(\tilde{\tau}+1)=k, \quad \mathrm{~d} B / \mathrm{d} \tilde{\eta}=k .
$$


The constant $k$ has to be obtained by an initial condition. Integration of the second equation is straightforward leading to a uniform gradient (straight profile of the flow). Introducing the initial value $\tau=0: A=A_{i}$, the solution of the first equation is,

$$
A^{-1}=(1+\tilde{\tau}) A_{i}^{-1}+k(1+\tilde{\tau}) \ln (1+\tilde{\tau})
$$

the first term on the right-hand side being the general solution of the homogeneous equation and the second term a particular solution. Solutions of (2.42) then take the form

$$
\begin{gathered}
\mu(\tilde{\xi}, \tilde{\tau})=\frac{k\left[\tilde{\xi}-\tilde{\xi}_{0}(\tilde{\tau})\right]}{(1+\tilde{\tau})\left[A_{i}^{-1}+k \ln (1+\tilde{\tau})\right]}-1 \\
\tilde{\xi}=0: \mu_{f}(\tilde{\tau})=\frac{-k \tilde{\xi}_{0}(\tilde{\tau})}{(1+\tilde{\tau})\left[A_{i}^{-1}+k \ln (1+\tilde{\tau})\right]}-1, \quad \tilde{\tau}=0: 1+\mu_{f i}=-k \tilde{\xi}_{0 i} A_{i}
\end{gathered}
$$

where the initial values $\mu_{f i}$ and $\tilde{\xi}_{0 i}$ have been used, $\tilde{\tau}=0: \mu_{f}=\mu_{f i}, \tilde{\xi}_{0}=\tilde{\xi}_{0 i}$. Eliminating $A_{i}$ in favour of $\mu_{f i}, A_{i}^{-1}=-k \tilde{\xi}_{0 i} /\left(1+\mu_{f i}\right)$, the constant $k$ is also eliminated from the expressions of $\mu(\tilde{\xi}, \tilde{\tau})$ and $\mu_{f}(\tilde{\tau})$, leading to a two-parameters family of solutions involving the parameters $\mu_{f i}$ and $\tilde{\xi}_{0 i}$,

$$
\begin{array}{rlrl}
\mu(\tilde{\xi}, \tilde{\tau}) & =\frac{\left[\tilde{\xi}-\tilde{\xi}_{0}(\tilde{\tau})\right]}{(1+\tilde{\tau})\left[\theta_{i}+\ln (1+\tilde{\tau})\right]}-1, & & \theta_{i} \equiv \frac{-\tilde{\xi}_{0 i}}{\left(1+\mu_{f i}\right)}>0 \\
\mu_{f}(\tilde{\tau})=\frac{-\tilde{\xi}_{0}(\tilde{\tau})}{(1+\tilde{\tau})\left[\theta_{i}+\ln (1+\tilde{\tau})\right]}-1, & \tilde{\xi}_{0}(\tilde{\tau})=-\left[\tilde{\tau}+\int_{0}^{\tilde{\tau}} \dot{\alpha}_{\tau}\left(\tilde{\tau}^{\prime}\right) \mathrm{d} \tilde{\tau}^{\prime}\right]+\tilde{\xi}_{0 i} .
\end{array}
$$

These expressions for the flow field are solutions to $(2.37)$ and also to $(2.42)$ when $\dot{\alpha}_{\tau}(\tilde{\tau})$ is small. Notice that the unknown function $\dot{\alpha}_{\tau}(\tilde{\tau})$ appears only through $\tilde{\xi}_{0}(\tilde{\tau})$. Written with the original variables, denoting $u_{f}(t)$ the flow on the front, $u_{f i}$ its initial value $(t=0)$, $r_{f i}$ the initial radius of the front and $\mathcal{D}_{i}>\mathcal{D}(t)$ the initial detonation velocity, equations (2.49)-(2.50), using the definition in (2.28) $1+\mu=u / \epsilon a$, take the form

$$
\begin{array}{ll}
0<\frac{u_{f i}}{\epsilon a}-1 \ll 1, & r_{0}(t) \leqslant r \leqslant r_{f}(t): \quad \frac{u(r, t)}{u_{f}(t)}=\frac{r-r_{0}(t)}{r_{f}(t)-r_{0}(t)} \\
r_{0}(t)=a t+r_{0 i}, & \frac{u_{f}(t)}{u_{f i}}=\frac{r_{f}(t)-r_{0}(t)}{r_{f i}+a t}\left[\frac{r_{f i}-r_{0 i}}{r_{f i}}+\frac{u_{f i}}{a} \ln \left(1+\frac{a t}{r_{f i}}\right)\right]^{-1}
\end{array}
$$

where $u_{f i} / a=\left(r_{f i}-r_{0 i}\right) /\left(r_{0 i} \theta_{i}\right)$. Self consistency of the asymptotic analysis in the limit $\epsilon \rightarrow 0 u_{f i} / a=O(\epsilon)$ is ensured by the scaling

$$
\epsilon \rightarrow 0: \quad \frac{r_{f}(t)-r_{0}(t)}{\epsilon r_{0}(t)}=-\frac{\tilde{\xi}_{0}(\tilde{\tau})}{\tilde{\tau}+1}=O(1) .
$$

Under the condition (2.41), the integral term can be neglected in the expression (2.50) of $\tilde{\xi}_{0}$, so that $(2.53)$ reduces to $(2.25)\left(r_{f}(t)-r_{0}(t)\right) / \epsilon r_{0}(t)=1$ for $\tilde{\xi}_{0 i}=-1$.

Notice also that $\mu(\tilde{\xi}, \tilde{\tau})$ in (2.49)-(2.52) can be written in the same form as the selfsimilar solution of $(2.18), \mathrm{v}=[z+\mathcal{A}(\nu)] / \mathcal{B}(\nu)$ with $z=\eta / \nu, \mathcal{B}(\nu)=\left(\theta_{i}+\ln \nu\right)$ and $\mathcal{A}(\nu)=\left[r_{f}(t)-r_{0}(t)\right] / \epsilon r_{0}(t)$ which reduces to $\mathcal{A}(\nu)=1-\left(1+\tilde{\xi}_{0 i}\right) / \nu$ for $\dot{\alpha}_{\tau} \ll 1$ and $\mathcal{A}(\nu)=1$ for $\tilde{\xi}_{0 i}=-1$.

\subsubsection{Simplified expression of the rarefaction flow near the CJ regime.}

According to (2.49) and/or (2.51), the gradient of the flow is uniform and decreases monotonously with the time. According to (2.50) and/or (2.52) the flow velocity on the 
front also decreases and the planar CJ velocity is reached at finite time $t_{t}$

$$
t=t_{t}: u_{f} / \epsilon a=1, \mathcal{D}=\mathcal{D}_{o_{C J}} \quad \Leftrightarrow \quad \tilde{\tau}=\tilde{\tau}_{t}: \mu_{f}=0, \tilde{\tau}_{t} \equiv a t_{t} / r_{f i}=\tau_{t} / \tilde{r}_{f i} .
$$

However at $t-t_{t}=0^{-}$, the flow field (2.49)-(2.52) is different from the self-similar rarefaction wave behind a CJ wave (2.22)-(2.24) plotted in Figure 1. We will show in $\S 3.1$ that the relaxation of the flow towards the CJ rarefaction wave occurs progressively for $t>t_{t}$ after a sudden and sharp transition of the flow gradient on the front at $t=t_{t}$.

At the end of the decay, $\left|\dot{\alpha}_{\tau}\right| \ll 1$, equation (2.50) where the integral term $\int_{0}^{\tilde{\tau}} \dot{\alpha}_{\tau}\left(\tilde{\tau}^{\prime}\right) \mathrm{d} \tilde{\tau}^{\prime}$ is neglected (initial condition close to the CJ velocity), introducing $\mu_{f}\left(\tilde{\tau}_{t}\right)=0$, leads to a transcendental equation for $\tilde{\tau}_{t}$ in terms of $\tilde{\xi}_{0 i}$ and $\mu_{f i}$

$\int_{0}^{\tau_{t}} \dot{\alpha}_{\tau}\left(\tau^{\prime}\right) \mathrm{d} \tau^{\prime} \ll \tau_{t}, \mu_{f}\left(\tilde{\tau}_{t}\right)=0 \Rightarrow \tilde{\tau}_{t}-\tilde{\xi}_{0 i}=\left(1+\tilde{\tau}_{t}\right)\left[-\frac{\tilde{\xi}_{0 i}}{\left(1+\mu_{f i}\right)}+\ln \left(1+\tilde{\tau}_{t}\right)\right]$,

which has a single positive root which is small for $\mu_{f i} \ll 1$ whatever $\tilde{\xi}_{0 i}<0$, see Appendix C.2. This root yields a simple expression of $\tilde{\tau}_{t}$ (and/or $t_{t}$ ) in terms of the initial value of the flow at the front

$$
\mu_{f i} \ll 1: \quad \tilde{\tau}_{t} \approx \mu_{f i} \ll 1 \Rightarrow \quad \tilde{\tau}_{t} \equiv \frac{a t_{t}}{r_{f i}} \approx\left(\frac{u_{f i}}{\epsilon a}-1\right) \ll 1, \quad \forall \tilde{\xi}_{0 i}<0
$$

obtained from the Taylor expansion of (2.55) when the quadratic terms $\tilde{\tau}_{t}^{2}$ are neglected. Limiting our attention to $0 \leqslant t \leqslant t_{t}, 0 \leqslant \tilde{\tau}=O\left(\tilde{\tau}_{t}\right)$, quadratic terms $\tilde{\tau}^{2}=\left(a t / r_{f i}\right)^{2}$ are negligible, and a Taylor expansion of (2.49)-(2.50) in powers of $\tilde{\tau}_{t} \approx \mu_{f i} \ll 1$, limited to first order, yields

$$
0 \leqslant \tilde{\tau} \leqslant \tilde{\tau}_{t} \ll 1: \quad \mu \approx \frac{\tilde{\xi}}{\left(-\tilde{\xi}_{0 i}\right)}\left[1+\tilde{\tau}_{t}-\left(1+\frac{1}{-\tilde{\xi}_{0 i}}\right) \tilde{\tau}\right]+\tilde{\tau}_{t}-\tilde{\tau}, \quad\left|\tilde{\xi}_{0 i}\right|=O(1) .
$$

The downstream condition at the weak discontinuity, $\xi=\xi_{0}(\tilde{\tau}): \mu=-1$ with $\tilde{\xi}_{0}=$ $-\tilde{\tau}+\tilde{\xi}_{0 i}$ in $(2.42)$, is recovered at the first order of the Taylor expansion of (2.57) in the form $\tilde{\xi}_{0} /\left(-\tilde{\xi}_{0 i}\right)=-1-\tilde{\tau} /\left(-\tilde{\xi}_{0 i}\right)$. According to (2.41) and (2.56), there is a boundary layer near the detonation front where the flow (2.57) takes an even simpler form at the end of the decay $0 \leqslant \tilde{\tau} \leqslant \tilde{\tau}_{t} \ll 1$

$$
\begin{aligned}
-\tilde{\xi} \equiv \frac{\left(r_{f}-r\right)}{\epsilon r_{f i}}=O\left(\tilde{\tau}_{t}\right), \quad \tilde{\tau}_{t} \approx \mu_{f i}=\sqrt{\frac{2\left(\mathcal{D}_{i}-\mathcal{D}_{o_{C I}}\right)}{\epsilon a}} \ll 1: \\
\mu(\tilde{\xi}, \tilde{\tau}) \approx \frac{\tilde{\xi}}{-\tilde{\xi}_{0 i}}-\left(\tilde{\tau}-\tilde{\tau}_{t}\right), \quad \mu_{f}(\tilde{\tau}) \approx\left(\tilde{\tau}_{t}-\tilde{\tau}\right) \\
\frac{u(r, t)}{\epsilon a}-1 \approx \frac{r-r_{f}(t)}{\epsilon r_{f i}}+\frac{a\left(t_{t}-t\right)}{r_{f i}}, \quad \frac{u_{f}(t)}{\epsilon a}-1 \approx \frac{a\left(t_{t}-t\right)}{r_{f i}},
\end{aligned}
$$

obtained when terms of second order $\tilde{\tau}_{t}^{2}$ are neglected. This leads to

$$
\frac{\partial \mu(\tilde{\xi}, \tilde{\tau})}{\partial \tilde{\tau}} \approx-1,\left.\quad \frac{\partial u(r, t)}{\partial r}\right|_{r=r_{f}} \approx \frac{a}{r_{f}}, \quad \frac{1}{\epsilon a} \frac{\mathrm{d} u_{f}(t)}{\mathrm{d} t} \approx-\frac{a}{r_{f}} \Rightarrow \frac{\mathrm{d} u_{f}(t)}{\mathrm{d} t} \approx-a \frac{u_{f}}{r_{f}},
$$

where $r_{f i}$ has been replaced by $r_{f}$ in the denominators since $r_{f}-r_{f i}=O\left(\epsilon r_{f}\right)$. The terms of order $\epsilon$ being neglected in (2.42), retaining the small term $\left(\tilde{\tau}_{t}-\tilde{\tau}\right)$ which is of order $\tilde{\tau}_{t} \approx \mu_{f i} \equiv\left(u_{f i} / \epsilon a\right)-1$, is meaningful in (2.59) in an intermediate asymptotic limit $\epsilon \ll \sqrt{2\left(\mathcal{D}_{i}-\mathcal{D}_{o_{C J}}\right) / \epsilon a} \ll 1$.

The last equation in (2.61) corresponds to the closure assumption used in Clavin \& 


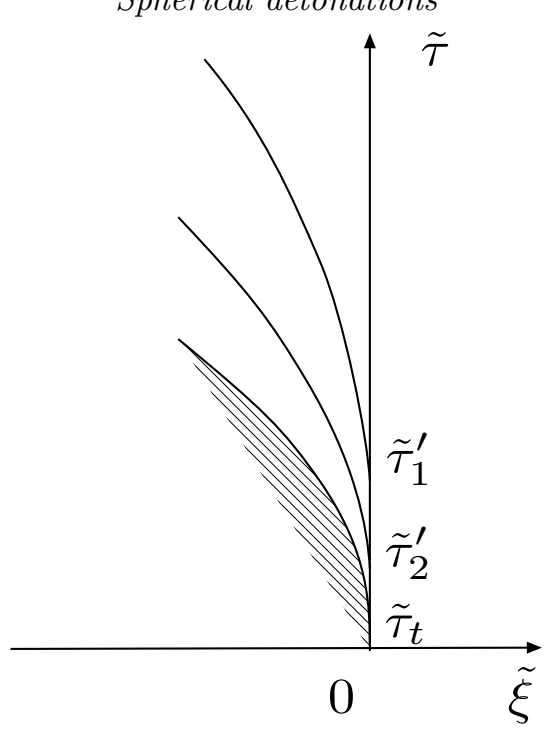

FiguRE 2. Example of three characteristic curves $\tilde{\xi}=\tilde{\xi}_{\mathcal{C}_{+}}\left(\tilde{\tau}, \tilde{\tau}^{\prime}\right)$ for $\tilde{\tau}^{\prime}=\tilde{\tau}_{t}, \tilde{\tau}^{\prime}=\tilde{\tau}_{2}^{\prime}$ and $\tilde{\tau}^{\prime}=\tilde{\tau}_{1}^{\prime}$ with $\tilde{\tau}_{t}<\tau_{2}^{\prime}<\tau_{1}^{\prime}$. In the dashed region $\tilde{\xi} \leqslant \tilde{\xi}_{\mathcal{C}_{+}}\left(\tilde{\tau}, \tilde{\tau}_{t}\right)$, the transitory flow is equal to the unperturbed flow (3.7) (straight profile whose slope is decreasing to zero) $\mu^{(t r)}(\tilde{\xi}, \tilde{\tau})=\mu^{(w d)}(\tilde{\xi}, \tilde{\tau})$.

Denet (2020) who neglected the small gradient of the flow at the exit of the reaction zone. This equation is indeed quite general close to the CJ regime and was derived previously in the opposite limit $M_{o_{C J}} \gg 1$ by Liñan et al. (2012). This can be seen in (2.9)-(2.10); because of the transonic character of the burnt gas flow near the detonation, $u+a-\mathcal{D} \ll 1$, the term involving the gradient of the flow on the left-hand side becomes negligible near the detonation front, the unsteady term being balanced by the divergence of the flow $-a u / r$. More precisely in the limit of small heat-release, using $\mathcal{D}_{o_{C J}}-a \approx \epsilon a$, $\mathcal{D}-a=\epsilon a\left(1+\dot{\alpha}_{\tau}\right) \approx \epsilon a, u_{f}=O(\epsilon a), \mu=u / \epsilon a-1$, see (2.28), and, according to (2.61), $\partial u /\left.\partial r\right|_{r=r_{f}} \approx a / r_{f}$ with $(2.56) \mu_{f}<\mu_{f i} \ll 1$, the gradient term on the left-hand side of (2.10) is shown to be smaller than the curvature term $a u / r$ on the right-hand side by a factor $\epsilon$,

$$
\left.\left(u_{f}+a-\mathcal{D}\right) \frac{\partial u}{\partial \mathrm{x}}\right|_{\mathrm{x}=0} \approx \epsilon \mu_{f} a \frac{a}{r_{f}} \ll a \frac{\epsilon a}{r_{f}}=O\left(\frac{a u_{f}}{r_{f}}\right) .
$$

As we shall see, this small gradient of the rarefaction flow cannot be ignored in the critical dynamics studied in $\S 4$ because it controls the instantaneous position of the sonic point inside the rarefaction wave.

\section{Transitory flow for the discontinuous model}

The way the rarefaction wave (2.49) reaches the self-similar solution behind the CJ wave (2.22)-(2.23) after $t_{t}$, is presented in this section for $M_{O_{C J}}-1 \ll 1$. The transition is similar to that described by Liñan et al. (2012) in the opposite limit $M_{o_{C J}} \gg 1$.

\subsection{Abrupt transition of the flow on the front}

Both the flows (2.49) and (2.22)-(2.23) vanish at the radius of the spherical core of stagnant gas but the gradient of the flow is uniform and finite in the former while it is infinite on the detonation front in the latter, see Figure 1. The sonic condition of (2.49) 


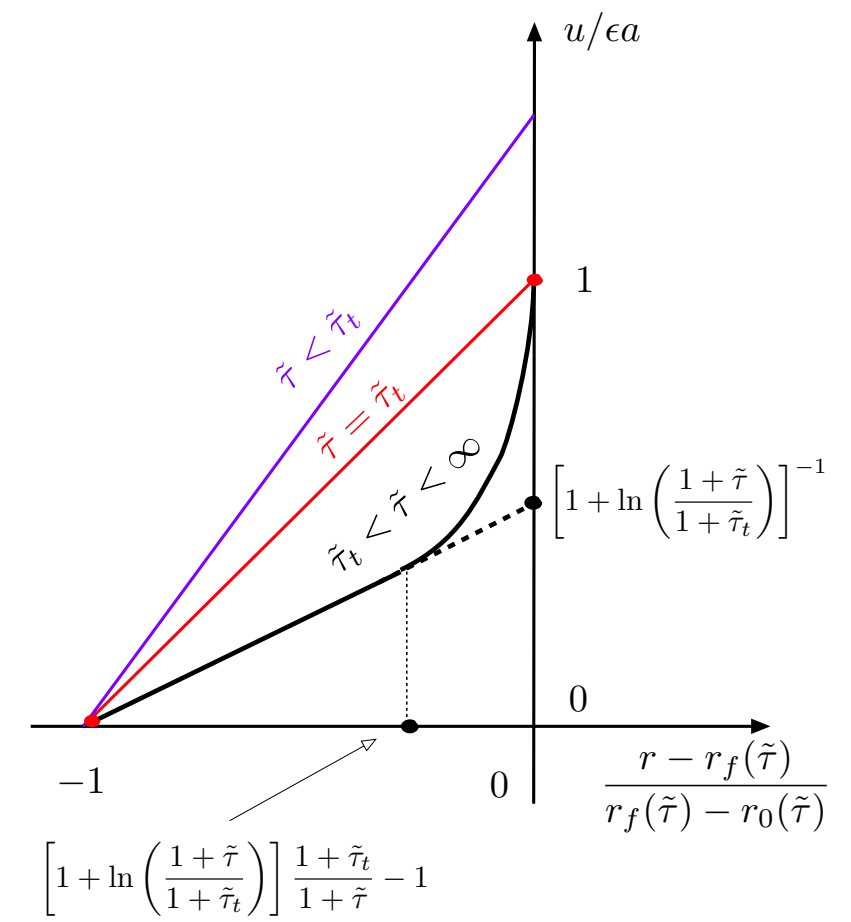

FIGURE 3. Sketch of the transitory flow, plotted in a universal form for $\tilde{\xi}_{0 i}=-1$ and $\tilde{\tau}_{t} \approx \mu_{f i} \ll 1, \tilde{\tau}_{t}$ being the cross-over time (2.55). The bold curve in black is the rarefaction wave $u^{(t r)} / \epsilon a=1+\mu^{(t r)}$ behind a detonation treated as a discontinuity, after the cross-over time $\tilde{\tau}>\tilde{\tau}_{t}$. The slope is infinite at the front and the profile is straight away from the front where $\mu^{(t r)}=\mu^{(w d)}$. Expressed in terms of the space variable $\tilde{\xi} /\left(-\tilde{\xi}_{0}\right)=\left[r-r_{f}(t)\right] /\left[r_{f}(t)-r_{0}(t)\right]$, $-\tilde{\xi}_{0}(\tilde{\tau})=\tilde{\tau}+1$, the profile of the transitory flow is parameter free, provided the time is measured by $(1+\tilde{\tau}) /\left(1+\tilde{\tau}_{t}\right)$. The bold points are the time dependent radius and flow velocity of the junction point where the transitory flow $\mu^{(t r)}$ matches $\mu^{w d}$ in (3.7). The colored straight lines represent the rarefaction wave (2.49) of the overdriven detonations before the velocity reaches the planar CJ velocity $\tilde{\tau}<\tilde{\tau}_{t}$, the red one being just before the transition to CJ, $\tilde{\tau}-\tilde{\tau}_{t}=0^{-}$.

(relative to the detonation front) which is located at finite distance behind the detonation front for $t<t_{t}$, reaches the front at $t=t_{t}$. Within the framework of the discontinuous model, the velocity of the burnt gas relative to the lead shock cannot become smaller than the sound speed on the detonation front. According to (2.40)-(2.41), neither $\dot{\alpha}_{t}(t)$ nor $\mu_{f}(t)$ can become negative; they should vanish simultaneously at $t=t_{t}$ and stay equal to zero at later times $t>t_{t}$.

$$
t>t_{t}: \quad u_{f}=\epsilon a, \quad \mu_{f}=0, \quad \mathcal{D}=\mathcal{D}_{o_{C J}}, \quad r_{f}(t)=\mathcal{D}_{o_{C J}}\left(t-t_{t}\right)+r_{f}\left(t_{t}\right)
$$

Therefore, the decrease of $u_{f}(t)$ with a quasi-constant deceleration rate $(2.60)$ for $t \leqslant t_{t}$ stops suddenly at $t=t_{t}$ since $u_{f}$ stays constant after $t_{t}$. A jump of deceleration of the flow is thus produced at $t=t_{t}$ on the detonation front

$$
\left.\frac{1}{\epsilon a} \frac{\mathrm{d} u_{f}}{\mathrm{~d} t}\right|_{t_{t}^{-}} ^{t_{t}^{+}}=\frac{a}{r_{f}\left(t_{t}\right)},\left.\quad \frac{\mathrm{d} \mu_{f}}{\mathrm{~d} \tilde{\tau}}\right|_{\tilde{\tau}_{t}^{-}} ^{\tilde{\tau}_{t}^{+}}=\frac{r_{f i}}{r_{f}\left(t_{t}\right)} \approx 1 .
$$


However, the trajectory in the phase space of velocity-radius " $\mathcal{D}-r_{f}$ " is tangent to the axis $\mathcal{D}=\mathcal{D}_{o_{C J}}$ at $t=t_{t}$, as shown by $(2.40) \dot{\alpha}_{\tau}=\mu_{f}^{2}(\tau) / 2$ by using $(2.60) \mu_{f} \approx a\left(t_{t}-t\right) r_{f i}$

$$
\begin{aligned}
\epsilon \ll 1,\left(t-t_{t}\right) \rightarrow 0^{-}: \quad & {\left[\mathcal{D}(t)-\mathcal{D}_{o_{C J}}\right] / a \rightarrow(\epsilon / 2)\left[\left(t-t_{t}\right) a / r_{f i}\right]^{2}, } \\
\mathrm{~d} r_{f} / \mathrm{d} t & \approx \mathcal{D}_{o_{C J}}=a(1+\epsilon), \quad\left(\mathcal{D}-\mathcal{D}_{o_{C J}}\right) / a \rightarrow(\epsilon / 2)\left(r_{f}-r_{f}\left(t_{t}\right)\right)^{2} / r_{f i}^{2},
\end{aligned}
$$

the last relation being valid near the CJ regime $0 \leqslant \mathcal{D}-\mathcal{D}_{o_{C J}} \ll \mathcal{D}_{o_{C J}}$ in the limit of small heat release $\left(\mathcal{D}_{o_{C J}}-a\right) / a=\epsilon \ll 1$. The jumps in (3.2) and the tangency of the trajectories at $t=t_{t}$ are consequences of the discontinuous model and are no longer valid when small modification of the inner structure of the detonation is taken into account.

\subsection{Transitory regime. An analytical study}

The transitory flow between the transition from the straight profile $(2.49)$ for $\tilde{\tau} \leqslant \tilde{\tau}_{t}$ to the self-similar solution $(2.22)-(2.24)$, denoted $\mu^{(t r)}(\tilde{\xi}, \tilde{\tau})$ in the following, is solution of (2.42) for a flow velocity at the front kept equal to its CJ value after $t_{t}$.

$$
\tilde{\tau} \geqslant \tilde{\tau}_{t}: \quad \frac{\partial \mu^{(t r)}}{\partial \tilde{\tau}}+\mu^{(t r)} \frac{\partial \mu^{(t r)}}{\partial \tilde{\xi}}=-\frac{1+\mu^{(t r)}}{\tilde{\tau}+1}, \quad \tilde{\xi}=0: \mu^{(t r)}=0 .
$$

This flow is equal to (2.49) (with $\mu_{f i}>0$ ) when $t<t_{t}$,

$$
\tilde{\tau} \approx \tilde{\tau}_{t}^{-}, \quad \tilde{\xi}_{0}\left(\tilde{\tau}_{t}\right) \leqslant \tilde{\xi} \leqslant 0: \quad \mu^{(t r)}\left(\tilde{\xi}, \tilde{\tau}_{t}\right)=\frac{\tilde{\xi}}{-\tilde{\xi}_{0 i}+\tilde{\tau}_{t}}=\frac{\tilde{\xi}}{-\tilde{\xi}_{0}\left(\tilde{\tau}_{t}\right)},
$$

where the simplified expression (2.57) has been used by introducing (2.55)-(2.56) into (2.49) neglecting the quadratic terms $\tilde{\tau}_{t}^{2}$. Equation (3.5) says simply that the transitory flow at $\tilde{\tau}=\tilde{\tau}_{t}$ has a straight profile corresponding to the reduced thickness $\left|\tilde{\xi}_{0}\left(\tilde{\tau}_{t}\right)\right|$ of the rarefaction wave. Simultaneously with the jump of deceleration (3.2), the flow gradient on the detonation front, which is finite for $\tilde{\tau}<\tilde{\tau}_{t}$, jumps at $\tilde{\tau}=\tilde{\tau}_{t}$ for becoming infinite at $\tilde{\tau}=\tilde{\tau}_{t}^{+}$and stays infinite afterwards $\tilde{\tau}>\tilde{\tau}_{t}$

$$
\tilde{\tau}=\tilde{\tau}_{t}^{-} \ll 1:\left.\frac{\partial \mu}{\partial \tilde{\xi}}\right|_{\tilde{\xi}=0^{-}}=\frac{1}{-\tilde{\xi}_{0}\left(\tilde{\tau}_{t}\right)}, \quad \tilde{\tau}>\tilde{\tau}_{t}: \lim _{\tilde{\xi} \rightarrow 0^{-}} \frac{\partial \mu^{(t r)}}{\partial \tilde{\xi}} \approx \frac{1}{\sqrt{-2 \tilde{\xi}}} .
$$

These expressions are obtained by the same method as in $\S 2.2 .2$ for the self-similar CJ solution (2.22)-(2.23). Recalling that no boundary condition is used on the front to derive (2.49)-(2.50), this flow is still solution of (2.42) for $\tilde{\tau}>\tilde{\tau}_{t}$ when $\mu_{f}(\tilde{\tau})$ decreases below zero. The corresponding flow, denoted $\mu^{(w d)}(\tilde{\xi}, \tilde{\tau})$ from now on, takes the form

$$
\tilde{\tau}_{t} \approx \mu_{f i} \ll 1, \quad \tilde{\tau} \geqslant \tilde{\tau}_{t}, \quad \mu^{(w d)}(\tilde{\xi}, \tilde{\tau}) \approx \frac{\tilde{\xi}+\tilde{\tau}-\tilde{\xi}_{0 i}}{(1+\tilde{\tau})\left[-\tilde{\xi}_{0 i} /\left(1+\tilde{\tau}_{t}\right)+\ln (1+\tilde{\tau})\right]}-1,
$$

where, neglecting the quadratic terms $\tilde{\tau}_{t}^{2}$, the flow at $\tau=\tau_{t}$ is given in $(3.5), \mu^{(w d)}\left(\tilde{\xi}, \tilde{\tau}_{t}\right)=$ $\mu_{\tilde{\xi}}^{(t r)}\left(\tilde{\xi}, \tilde{\tau}_{t}\right)$. The instantaneous position of the weak discontinuity corresponding to (3.7), $\tilde{\xi}=\tilde{\xi}_{0}(\tilde{\tau}): 1+\mu^{(w d)}\left(\tilde{\xi}_{0}, \tilde{\tau}_{t}\right)=0$, takes the form

$$
\tilde{\xi}_{0}(\tilde{\tau})=\tilde{\xi}_{0}\left(\tilde{\tau}_{t}\right)-\left(\tilde{\tau}-\tilde{\tau}_{t}\right)
$$

Equation (3.7) cannot represent the transitory flow nearby the detonation front for $\tilde{\tau}>$ $\tilde{\tau}_{t}: \mu^{(w d)}(\tilde{\xi}, \tilde{\tau}) \neq \mu^{(t r)}(\tilde{\xi}, \tilde{\tau})$ since $\mu^{(t r)}(0, \tilde{\tau})=0$ while $\mu^{(w d)}(0, \tilde{\tau})<0 \forall \tilde{\tau}>\tilde{\tau}_{t}$. However (3.7) still represents the transitory flow at a distance from the front large enough, as explained now by the method of characteristics.

The singular perturbation (3.6), which is generated instantaneously at $\tilde{\tau}=\tilde{\tau}_{t}$ on the front, induces a disturbance which is transported downstream with the velocity $\mu^{(t r)}$ by 
the mode $\mathcal{C}_{+}$(downstream-running for $\tilde{\tau}>\tilde{\tau}_{t}$ since $\mu^{(t r)} \leqslant 0$ ). According to (3.4), the characteristic curves $\tilde{\xi}=\tilde{\xi}_{\mathcal{C}_{+}}(\tilde{\tau})$

$$
\mathrm{d} \tilde{\xi}_{\mathcal{C}_{+}} / \mathrm{d} \tilde{\tau}=\mu^{(t r)}\left(\tilde{\xi}_{\mathcal{C}_{+}}(\tilde{\tau}), \tilde{\tau}\right)
$$

corresponds to the following equation for the flow velocity transported by $\mathcal{C}^{+}$

$$
\mu_{\mathcal{C}_{+}}^{(t r)}(\tilde{\tau}) \equiv \mu^{(t r)}\left(\tilde{\xi}_{\mathcal{C}_{+}}(\tilde{\tau}), \tilde{\tau}\right), \quad \frac{\mathrm{d} \mu_{\mathcal{C}_{+}}^{(t r)}(\tilde{\tau})}{\mathrm{d} \tilde{\tau}}=-\frac{1+\mu_{\mathcal{C}_{+}}^{(t r)}(\tilde{\tau})}{\tilde{\tau}+1}
$$

Denoting $\tilde{\xi}=\tilde{\xi}_{\mathcal{C}_{+}}\left(\tilde{\tau}, \tilde{\tau}^{\prime}\right)$ the characteristic curve leaving the front $(\tilde{\xi}=0)$ at $\tilde{\tau}=\tilde{\tau}^{\prime} \geqslant \tilde{\tau}_{t}$, integration of $(3.10)$ from $\tilde{\tau}=\tilde{\tau}^{\prime}: \mu_{\mathcal{C}_{+}}^{(t r)}\left(\tilde{\tau}^{\prime}\right)=0$ yields the flow velocity transported by this characteristic in the form

$$
\tilde{\tau} \geqslant \tilde{\tau}^{\prime} \geqslant \tilde{\tau}_{t}: \quad 1+\mu^{(t r)}\left(\tilde{\xi}_{\mathcal{C}_{+}}\left(\tilde{\tau}, \tilde{\tau}^{\prime}\right), \tilde{\tau}\right)=\frac{\tilde{\tau}^{\prime}+1}{\tilde{\tau}+1} .
$$

Integrating (3.9) from $\tau=\tau^{\prime}: \tilde{\xi}_{\mathcal{C}_{+}}\left(\tilde{\tau}^{\prime}, \tilde{\tau}^{\prime}\right)=0$ using (3.11), then determines the characteristic curves $\tilde{\xi}=\tilde{\xi}_{\mathcal{C}_{+}}\left(\tilde{\tau}, \tilde{\tau}^{\prime}\right)$

$$
\begin{aligned}
\tilde{\tau} \geqslant \tilde{\tau}^{\prime} \geqslant \tilde{\tau}_{t}: \quad \frac{\partial \tilde{\xi}_{\mathcal{C}_{+}}\left(\tilde{\tau}, \tilde{\tau}^{\prime}\right)}{\partial \tilde{\tau}} & =\frac{\tilde{\tau}^{\prime}+1}{\tilde{\tau}+1}-1 \leqslant 0 \\
\tilde{\xi}_{\mathcal{C}_{+}}\left(\tilde{\tau}, \tilde{\tau}^{\prime}\right) & =\left(\tilde{\tau}^{\prime}+1\right) \ln \frac{\tilde{\tau}+1}{\tilde{\tau}^{\prime}+1}-\left(\tilde{\tau}-\tilde{\tau}^{\prime}\right) .
\end{aligned}
$$

All these characteristics leave the front slowly since their velocity is zero at the front $(\xi=0) \mu^{(t r)}\left(0, \tilde{\tau}^{\prime}\right)=0, \partial \tilde{\xi}_{\mathcal{C}_{+}}\left(\tilde{\tau}, \tilde{\tau}^{\prime}\right) /\left.\partial \tilde{\tau}\right|_{\tilde{\tau}=\tilde{\tau}^{\prime}}=0 \forall \tilde{\tau}^{\prime} \geqslant \tilde{\tau}_{t}$. They all reach the same velocity in the long-time $\operatorname{limit}, \lim _{\tilde{\tau} \rightarrow \infty} \partial \tilde{\xi}_{\mathcal{C}_{+}}\left(\tilde{\tau}, \tilde{\tau}^{\prime}\right) / \partial \tilde{\tau}=-1$. Moreover, in agreement with the fact that no shock can be created when the flow $u(r, t)$ increases in space in the direction of the flow, $\partial u / \partial r>0$, these characteristic curves do not cross each other. This is checked by noticing that the modulus of their velocity satisfies the following relation $\tilde{\tau}_{1}^{\prime}>\tilde{\tau}_{2}^{\prime} \Rightarrow\left|\partial \tilde{\xi}_{\mathcal{C}_{+}}\left(\tilde{\tau}, \tilde{\tau}_{1}^{\prime}\right) / \partial \tilde{\tau}\right|<\left|\partial \tilde{\xi}_{\mathcal{C}_{+}}\left(\tilde{\tau}, \tilde{\tau}_{2}^{\prime}\right) / \partial \tilde{\tau}\right|$, see Figure 2. Therefore, the trajectory $\tilde{\xi}=\tilde{\xi}_{\mathcal{C}_{+}}\left(\tilde{\tau}, \tilde{\tau}_{t}\right)$ associated with the characteristic curve leaving the front at the earliest time $\tilde{\tau}^{\prime}=\tilde{\tau}_{t}$ is, according to (3.13),

$$
\begin{aligned}
\tilde{\tau} \geqslant \tilde{\tau}_{t}, \quad \tilde{\xi}_{\mathcal{C}_{+}}\left(\tilde{\tau}, \tilde{\tau}_{t}\right) & =\left(\tilde{\tau}_{t}+1\right) \ln \frac{\tilde{\tau}+1}{\tilde{\tau}_{t}+1}-\left(\tilde{\tau}-\tilde{\tau}_{t}\right), \\
& =\left(\tilde{\tau}_{t}+1\right) \ln (\tilde{\tau}+1)-\tilde{\tau}+O\left(\tilde{\tau}_{t}^{2}\right),
\end{aligned}
$$

with, according to (3.11), the following expression of the flow which is transported

$$
\mu^{(t r)}\left(\tilde{\xi}_{\mathcal{C}_{+}}\left(\tilde{\tau}, \tilde{\tau}_{t}\right), \tilde{\tau}\right)=\frac{\tilde{\tau}_{t}+1}{\tilde{\tau}+1}-1 .
$$

In other words, $\tilde{\xi}=\tilde{\xi}_{\mathcal{C}_{+}}\left(\tilde{\tau}, \tilde{\tau}_{t}\right)$ is the equation of the leading edge of the disturbance resulting from keeping equal to zero the front velocity $\tilde{\xi}=0: \mu^{(t r)}=0 \forall \tilde{\tau}>\tilde{\tau}_{t}$. Ahead of this leading edge, the flow $\mu^{(t r)}(\tilde{\xi}, \tilde{\tau})$ is equal to the "unperturbed" flow $(3.7)$,

$$
\tilde{\xi} \leqslant \tilde{\xi}_{\mathcal{C}_{+}}\left(\tilde{\tau}, \tilde{\tau}_{t}\right): \quad \mu^{(t r)}(\tilde{\xi}, \tilde{\tau})=\mu^{(w d)}(\tilde{\xi}, \tilde{\tau}) .
$$

The junction point at which $\mu^{(w d)}(\tilde{\xi}, \tilde{\tau})$ is equal to $(3.16)$

$$
\frac{\tilde{\xi}(\tilde{\tau})+\tilde{\tau}-\tilde{\xi}_{0 i}}{-\tilde{\xi}_{0 i} /\left(1+\tilde{\tau}_{t}\right)+\ln (1+\tilde{\tau})}=\tilde{\tau}_{t}+1 \Rightarrow \tilde{\xi}(\tilde{\tau})=-\tilde{\tau}+\left(\tilde{\tau}_{t}+1\right) \ln (1+\tilde{\tau}) \quad \forall \tilde{\xi}_{0 i}
$$


corresponds effectively to the characteristics (3.14) when the quadratic terms $\tilde{\tau}_{t}^{2}$ are neglected as it should be.

Strictly speaking, this point does not reach the core of stagnant gas (3.8) $\tilde{\xi}_{0}(\tilde{\tau})$ in the long time limit, $\lim _{\tilde{\tau} \rightarrow \infty} \tilde{\xi}_{\mathcal{C}_{+}}\left(\tilde{\tau}, \tilde{\tau}_{t}\right)-\tilde{\xi}_{0}(\tilde{\tau}) \approx \ln \tilde{\tau}$. However, this is true in the sense of the self-similar analysis when using the self-similar variable $z_{\mathcal{C}_{+}}(\tilde{\tau}) \equiv\left[r_{\mathcal{C}_{+}}(t)-r_{f}(t)\right] / \epsilon r_{0}(t) \approx$ $\tilde{\xi}_{\mathcal{C}_{+}}\left(\tilde{\tau}, \tilde{\tau}_{t}\right) /(-\tilde{\tau})$, yielding, according to $(3.15)$

$$
\lim _{\tilde{\tau} \rightarrow \infty} \tilde{\xi}_{\mathcal{C}_{+}}\left(\tilde{\tau}, \tilde{\tau}_{t}\right) / \tilde{\xi}_{0}(\tilde{\tau})=1, \quad \lim _{\tilde{\tau} \rightarrow \infty} z_{\mathcal{C}_{+}}(\tilde{\tau})=-1,
$$

To conclude, the transitory flow $\mu^{(t r)}(\tilde{\xi}, \tilde{\tau})$ is composed of two parts. The part corresponding to $\tilde{\xi} \leqslant \tilde{\xi}_{\mathcal{C}_{+}}\left(\tilde{\tau}, \tilde{\tau}_{t}\right)$ is the straight profile $\mu^{(w d)}(\tilde{\xi}, \tilde{\tau})$ in (3.7) whose slope is uniform and decreases to zero $\lim _{\tilde{\tau} \rightarrow \infty}(1+\tilde{\tau})^{-1}\left[-\tilde{\xi}_{0 i} /\left(1+\tilde{\tau}_{t}\right)+\ln (1+\tilde{\tau})\right]^{-1}=0$. The transitory flow has a curved shape in the range $\xi_{\mathcal{C}_{+}}\left(\tilde{\tau}, \tilde{\tau}_{t}\right) \leqslant \tilde{\xi} \leqslant 0$, joining the straight solution $\mu^{(w d)}(\tilde{\xi}, \tilde{\tau})$ at $\tilde{\xi}=\tilde{\xi}_{\mathcal{C}_{+}}\left(\tilde{\tau}, \tilde{\tau}_{t}\right)$, while the slope is infinite at the front $(\tilde{\xi}=0)$. The evolution of the rarefaction wave is sketched in figure 3 where the theoretical expressions for the coordinates (position and flow velocity at time $t$ ) of the point of junction with the straight profile are given.

These theoretical results are fully confirmed by the numerical solution of (3.4)-(3.5). An example of numerical result is shown in figure 4 . When the result is plotted with a space coordinate reduced by the thickness of the CJ rarefaction wave, the numerical analysis shows that the transitory flow and the self-similar CJ solution are quasi-identical in the range between the detonation front and the junction point traveling with the characteristic $\mathcal{C}_{+}$issued from the front at $t=t_{t}$ at which the front reaches the CJ velocity. Outside this range, the numerical result shows a straight profile down to the stagnant core, in full agreement with (3.7). The trajectory of the junction point between the two parts of the transitory flow corresponds exactly to the theoretical result plotted in figure 3 .

\section{Direct initiation of detonation}

Modification to the inner structure influences drastically the dynamics of a spherical detonation. In contrast to the discontinuous model, detonation failure is observed when the detonation velocity reaches the CJ velocity for the first time at a too small radius (however larger than the detonation thickness). Moreover, during successful initiation of a stable detonation, the CJ velocity is no longer reached abruptly. The critical dynamics is analyzed here in the limit of small heat release.

\subsection{Detonation model and method of solution}

The reduced variables of order unity governing the structure and the dynamics of the inner structure in the limit of small heat release $\epsilon \rightarrow 0$ are $\xi=O(1)$ and $\tau=O(1)$ in (2.27) where $r_{f i}$ is the initial radius of the detonation at $\tau=0$. According to (2.35)(2.36), the link of $\xi, \tau$ with $\tilde{\xi} \equiv \xi / \tilde{r}_{f i}$ and $\tilde{\tau}=\tau / \tilde{r}_{f i}$ is through the parameter $\tilde{r}_{f i} \equiv \epsilon r_{f i} / l$ of order unity in the limit $\epsilon \rightarrow 0$.

As in Clavin \& Denet (2020), we will use the detonation model of Clavin \& Williams (2002), obtained by an asymptotic analysis in the limit of small heat release, coupled to the Newtonian approximation neglecting the compressional heating in the reaction zone where the chemical energy is released (after the induction period),

$$
\epsilon \equiv \sqrt{q_{m} / c_{p} T_{u}} \approx M_{o_{C J}}-1 \ll 1, \quad(\gamma-1)<\epsilon .
$$




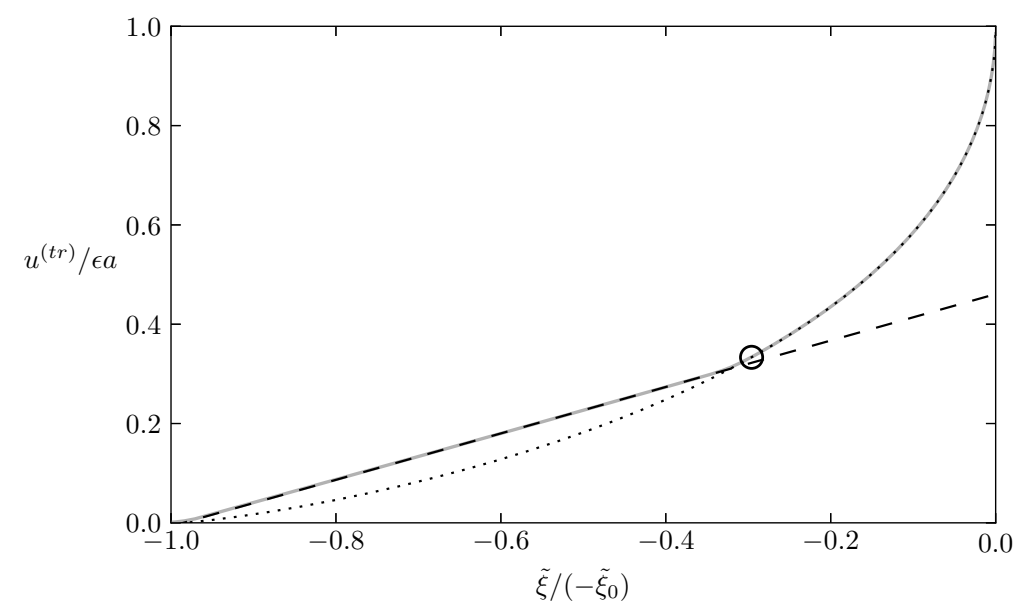

Figure 4. Numerical solution (solid line) of (3.4)-(3.5), describing the transitory flow $u^{(t r)} / \epsilon a=1+\mu^{(t r)}$ for $\tilde{\tau}-\tilde{\tau}_{t}=3$, using the non dimensional radius, reduced by the thickness of the rarefaction wave. The dotted line is the self-similar rarefaction flow (2.23)-(2.25) behind the spherical CJ detonation which is reached in the long time limit $\tilde{\tau}-\tilde{\tau}_{t} \rightarrow \infty$. The dashed straight line is (3.7) for $\tilde{\tau}-\tilde{\tau}_{t}=3$. The trajectory of the point at the junction of the numerical solution of (3.4)-(3.5) and the straight profile (3.7) is in full agreement with the theoretical prediction (3.12)-(3.18). To summarize, the numerical solution of (3.4)-(3.5) is found to be exactly the straight profile (3.7) below the junction while, in the range between the front and the junction point, there is no noticeable difference between the numerical solution of (3.4)-(3.5) and the self-similar solution (2.23)-(2.25).

The Rankine-Hugoniot conditions (A 3)-(A 5) in the limit (4.1) takes the form,

$$
\begin{aligned}
& T_{N} / T_{u} \approx 1+2(\gamma-1)(M-1), \quad b \equiv 2 \epsilon(\gamma-1) \mathcal{E} / k_{B} T_{u}, \\
& T_{N o_{C J}} / T_{u} \approx 1+2(\gamma-1) \epsilon, \quad\left(E / k_{B} T_{u}^{2}\right)\left(T_{N}-T_{N o_{C J}}\right)=b \dot{\alpha}_{\tau}
\end{aligned}
$$

where $\mathcal{E}$ is the activation energy governing the induction length, and $b=O(1)$ is its reduced form. A first simplification concerns the temperature and the mass fraction of species which, to leading order in the limit (4.1), are solutions of the steady-state version of the conservation equations of energy and mass, satisfying the Rankine-Hugoniot conditions at the lead shock, see the original article Clavin \& Williams (2002) recalled in § B.1 of Clavin \& Denet (2018). The unsteady distribution of the rate of heat release $\omega(\xi, \tau)$ can then be expressed in terms of the unsteady detonation velocity and the distribution in steady state, $\omega(\xi, \tau)=\bar{\omega}\left(\xi, \dot{\alpha}_{\tau}(\tau)\right)$. Focusing our attention on propagation velocity close enough to the CJ velocity, $y(\tau) \equiv b \dot{\alpha}_{\tau}(\tau)=O(1)$, the unsteady distribution of reaction rate takes the form $\omega(\xi, \tau)=\bar{\omega}(\xi, y(\tau))$ which will be denoted simply $\omega(\xi, y)$ in the following. For the sake of simplicity we will use here the scaling law assuming that the distribution of reaction rate is fully governed by the induction length. Introducing the distribution of the planar CJ wave $\omega_{O_{C J}}(\xi)$, the reaction-rate-distribution $\omega(\xi, y)$ then takes the form

$$
\omega(\xi, y)=\mathrm{e}^{y(\tau)} \omega_{o_{C J}}\left(\xi \mathrm{e}^{y(\tau)}\right), \quad y(\tau) \equiv b \dot{\alpha}_{\tau}(\tau)=\frac{b\left(\mathcal{D}(\tau)-\mathcal{D}_{o_{C J}}\right)}{\epsilon}=O(1) .
$$

The chemical-kinetics thus appears only through the activation energy $b$ and the CJ distribution $\omega_{o_{C J}}(\xi)$.

In order to further simplify the presentation, the same bounded-thickness model for 
the inner structure of the planar CJ detonation as in (3.15)-(3.17b) of Clavin \& Denet (2020) will be used,

$$
\begin{gathered}
\xi \leqslant-1: \omega_{O_{C J}}(\xi)=0, \quad \xi>-1: \omega_{O_{C J}}(\xi)>0 \\
\int_{-1}^{0} \omega_{O_{C J}}\left(\xi^{\prime}\right) \mathrm{d} \xi^{\prime}=1, \quad \mathrm{~d} \omega_{O_{C J}} /\left.\mathrm{d} \xi\right|_{\xi+1=0^{+}} \geqslant 0,
\end{gathered}
$$

so that, in the limit of small heat release, the structure of the flow in the planar CJ wave $(y=0) \mu_{o_{C J}}(\xi)$ is solution to

$$
\mu_{O_{C J}} \frac{\mathrm{d} \mu_{o_{C J}}}{\mathrm{~d} \xi}=\frac{1}{2} \omega_{O_{C J}}(\xi), \quad \xi \leqslant-1: \mu_{O_{C J}}=0, \quad \xi=0: \mu_{O_{C J}}=1 .
$$

According to (4.4) and (4.5) the exit of the reaction zone $\xi=\xi_{b}(\tau)$ of the unsteady inner structure of the spherical combustion wave is

$$
\xi_{b}(\tau)=-\mathrm{e}^{-y(\tau)},
$$

describing a substantial increase of the reaction-wave thickness when the velocity decreases below the CJ velocity $y<0$. However for a reduced detonation velocity of order unity $|y|=O(1)$, the thickness of the detonation is of the same order of magnitude as in the planar CJ wave, $\mathrm{e}^{-y}=O(1)$ in the limit $\epsilon \rightarrow 0$. The variation of $y$ is bounded from below since a supersonic velocity $\mathcal{D} \geqslant a$ implies $\dot{\alpha}_{\tau} \equiv\left(\mathcal{D}-\mathcal{D}_{o_{C J}}\right) / \epsilon a=(\mathcal{D}-a) / \epsilon a-1 \geqslant-1$ so that $y \equiv b \dot{\alpha}_{\tau} \geqslant-b$. In fact, according to the well-known chemical-kinetics quenching in combustion, occurring at a crossover temperature for which the recombination reactions become faster than the chain-branching reactions, the rate of heat release vanishes earlier. In real combustion, this crossover temperature $T_{c}$ at which the reaction rate decreases sharply and the induction time increases strongly is typically $T_{c} \approx 1000 \mathrm{~K}$ in ordinary condition while the Neumann temperature of a CJ wave is about $T_{N o C J} \approx 2000$ K, see Sanchez \& Williams (2014) and Clavin \& Searby (2016). Considering that ordinary combustion cannot proceed below $T_{c}$ with $T_{N o C J}-T_{c} \approx\left(T_{N o C J}-T_{u}\right) / 2$, a chemical quenching is assumed to be produced in the limit of small heat release for $y=y_{c}$ with typically $y_{c}=-b / 2$. Then, the following condition is added to (4.4)-(4.5)

$$
y \leqslant y_{c} \approx-b / 2: \quad \omega(\xi, y)=0 \quad \forall \xi .
$$

The reaction being quenched below $y \leqslant y_{c}$, detonation fails systematically as soon as the detonation velocity decreases below the lower bound $y_{c}$.

Another key simplification of the limit (4.1) concerns the two-timescale-nature of the dynamics which, to a lesser extent, characterizes also real detonations. To leading order in the limit (4.1), following the reasoning used in $\S 2.1$, the unsteady flow inside the inner structure of a spherical detonation is governed by a single equation corresponding to (2.35) plus an additional term on the right-hand side associated with the reaction rate

$$
\begin{gathered}
\epsilon \rightarrow 0: \quad \frac{\partial \mu}{\partial \tau}+\left(\mu-\frac{y}{b}\right) \frac{\partial \mu}{\partial \xi}=\frac{1}{2} \omega(\xi, y(\tau))-\frac{(1+\mu)}{\tilde{r}_{f}(\tau)}, \\
\xi=0: \quad \mu=1+2 y(\tau) / b .
\end{gathered}
$$

with, according to (2.30)-(2.32),

$$
\epsilon \rightarrow 0: \quad \tilde{r}_{f}(\tau) \equiv \epsilon r_{f}(\tau) / l \approx \tau+\tilde{r}_{f i} .
$$

The key point which is used to obtain (4.10) is that the variation of pressure and flow velocity across the inner structure of the reaction wave are identical to leading order in the limit (4.1), $\pi=\mu+1$ where $\pi \equiv(1 / \epsilon \mu), \ln \left(p / p_{u}\right) \approx u / a$, see Clavin \& Williams 


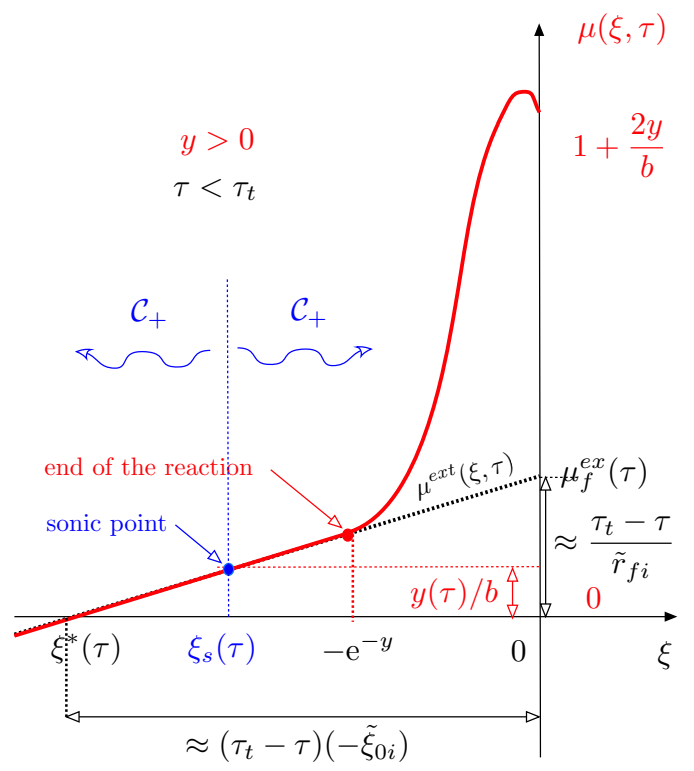

FiguRE 5. Sketch of the flow in an overdriven regime with $0<y / b<\mu_{f}^{e x t}, \mu^{e x t}\left(\xi_{s}, 0\right)=y / b$ $<\mu_{f}^{e x t}, \xi_{s}<-\mathrm{e}^{-y}$. The distances plotted on the horizontal and vertical axis correspond to the simplified expressions (C 11), $\tau_{t}$ corresponding to the time at which $\mu_{f}^{e x t}=0$.

(2002) and also § B.1 of Clavin \& Denet (2018) and § 3.1 of Clavin \& Denet (2020) for a spherical geometry. The boundary condition at the lead shock (4.11) is the RankineHugoniot relation (4.2)-(4.3) obtained from (A 5)-(A 6) for $M-1=\epsilon(1+y / b)$. The last term on the right-side of $(4.10)$ is the damping rate $(2.35)(l / a)(u / r)$ due to the divergence of the burnt-gas flow near the front for $r_{f} \gg l$ in a spherical geometry, $u / r \approx u / r_{f}$.

When the attention is focused on the inner structure, $\xi=O(1)$, the time-dependent velocity of the lead shock $y(\tau)$ is obtained as an eigenfunction of the system (4.10)(4.11) plus a boundary condition at the exit of the reaction zone. In contrast to Clavin $\&$ Denet (2020) where, considering the burnt-gas flow as uniform, the decelerating flow $\mu_{b}(\tau)$, solution to $\mathrm{d} \mu_{b} / \mathrm{d} \tau \approx-\left(1+\mu_{b}\right) / \tilde{r}_{f}$, was imposed in the burnt gas $\xi=-\mathrm{e}^{-y}$, the solution of (4.10)-(4.11) will be matched now with the non uniform flow of the rarefaction wave, solution to the inert version $(2.35)$ of $(4.10)$, denoted $\mu^{\text {ext }}(\xi, \tau)$ from now on. In overdriven regimes, this external flow field $\mu^{e x t}(\xi, \tau)$ is given by the analytical expressions (2.49)-(2.50), as discussed now.

\subsection{Overdriven regimes. Splitting and matching}

The burnt-gas flow at the exit of the reaction zone of a supersonic reaction wave in the overdriven regime is, by definition, subsonic relative to the lead shock, $\mathcal{D}-u_{b}<a \Leftrightarrow$ $\mu^{e x t}\left(-\mathrm{e}^{-y(\tau)}, \tau\right)>y(\tau) / b$. The sonic point $\xi=\xi_{s}(\tau), \mu^{e x t}\left(\xi_{s}(\tau), \tau\right)=y(\tau) / b$, is located inside the rarefaction flow, behind the inner structure of the combustion wave. Overdriven regimes are then characterized by the relations

$$
\xi_{s}(\tau)<-\mathrm{e}^{-y(\tau)}, \quad \mu^{e x t}\left(\xi_{s}(\tau), \tau\right)=y(\tau) / b, \quad \mu^{e x t}\left(-\mathrm{e}^{-y(\tau)}, \tau\right)>y(\tau) / b,
$$

the last inequality being in agreement with a rarefaction flow of inert gas which is an increasing function of the radius $\partial \mu^{e x t} / \partial \xi>0$. The larger the overdrive factor $\mu^{e x t}\left(-\mathrm{e}^{-y(\tau)}, \tau\right)-y(\tau) / b>0$ is, the larger is the distance between the sonic point and the exit of the reaction zone. The characteristic $\mathcal{C}_{+}$of equation $(2.35)$ is downstream 
running (towards the weak discontinuity $\left.\xi=\xi_{0}<0\right)$ behind the sonic point $\left(\xi<\xi_{s}\right.$, $\left.\mu^{\text {ext }}<y / b\right)$ ) while it is upstream running (towards the lead shock $\xi=0$ ) for $\xi>\xi_{s}$, $\mu^{e x t}>y / b$, see figure 5. Then, under the condition (4.13), modifications of the inner structure of the combustion wave cannot influence the rarefaction flow in the burnt gas which is still given by (2.49)-(2.50),

$$
\begin{aligned}
\xi \leqslant-\mathrm{e}^{-y(\tau)}: \mu(\xi, \tau) & =\mu^{e x t}(\xi, \tau), \\
\mu^{e x t}(\xi, \tau) & =\frac{\xi}{\tilde{r}_{f}(\tau)\left[\left(-\tilde{\xi}_{0 i}\right) /\left(1+\mu_{f i}^{e x t}\right)+\ln \left(\tilde{r}_{f} / \tilde{r}_{f i}\right)\right]}+\mu_{f}^{e x t}(\tau),
\end{aligned}
$$

where $\tilde{r}_{f}(\tau)$ is the reduced radius (4.12). Introducing the notation

$$
Y(\tau) \equiv(1 / \tau) \int_{0}^{\tau} \dot{\alpha}_{\tau}\left(\tau^{\prime}\right) \mathrm{d} \tau^{\prime}=O(y / b)
$$

the function $\mu_{f}^{e x t}(\tau)$ is, according to $(2.50)$,

$$
\mu_{f}^{e x t}(\tau)=\frac{\tau[1+Y(\tau)]+\left(-\tilde{\xi}_{0 i}\right) \tilde{r}_{f i}}{\tilde{r}_{f}(\tau)\left[\left(-\tilde{\xi}_{0 i}\right) /\left(1+\mu_{f i}^{e x t}\right)+\ln \left(\tilde{r}_{f} / \tilde{r}_{f i}\right)\right]}-1 .
$$

The external flow $\mu^{e x t}(\xi, \tau)$ is a linear function of the radius (straight shape) with a slope decreasing monotonously to zero, regardless of the time-dependent velocity of the front $y(\tau)$. The dependence of $\mu_{f}^{e x t}(\tau)$ on the past of the unknown solution $y(\tau)$ through the integral $Y(\tau)$ comes from the increase of the thickness of the rarefaction wave with the time, $-\xi_{0}(\tau) \equiv\left[r_{f}(\tau)-r_{0}(\tau)\right] / l,-\mathrm{d} \xi_{0} / \mathrm{d} \tau=1+y / b$, see (2.33)-(2.34). At large radius and close to the CJ velocity the external flow takes the simplified form (C11), reminiscent of (2.60). To summarize, for overdriven regimes, the boundary condition in the burnt gas of the solution of (4.10) is (4.14)

$$
\begin{array}{cl}
\xi_{s}(\tau)<-\mathrm{e}^{-y(\tau)} ; & \xi \leqslant-\mathrm{e}^{-y(\tau)}: \mu(\xi, \tau)=\mu^{e x t}(\xi, \tau) \\
\text { and, in particular } & \xi=-\mathrm{e}^{-y(\tau)}: \mu(\xi, \tau)=\mu^{e x t}\left(-\mathrm{e}^{-y(\tau)}, \tau\right) .
\end{array}
$$

Introducing the decomposition

$$
\mu(\xi, \tau)=\mu^{e x t}(\xi, \tau)+\hat{\mu}(\xi, \tau)
$$

and subtracting (2.35) from (4.10)

$$
\frac{\partial \hat{\mu}}{\partial \tau}+\left(\mu-\frac{y}{b}\right) \frac{\partial \mu}{\partial \xi}-\left(\mu^{e x t}-\frac{y}{b}\right) \frac{\partial \mu^{e x t}}{\partial \xi}=\frac{1}{2} \mathrm{e}^{y(\tau)} \omega_{o_{C J}}\left(\xi \mathrm{e}^{y(\tau)}\right)-\frac{\hat{\mu}}{\tilde{r}_{f}}
$$

the dynamics of the lead shock $y(\tau)$ during the decay of a combustion wave in the overdriven regime corresponds to the eigenfunction of the following problem

$$
\begin{aligned}
& \tau \leqslant \tau_{s}: \quad \frac{\partial \hat{\mu}}{\partial \tau}+\left(\hat{\mu}-\frac{y}{b}\right) \frac{\partial \hat{\mu}}{\partial \xi}=\frac{1}{2} \mathrm{e}^{y(\tau)} \omega_{o_{C J}}\left(\xi \mathrm{e}^{y(\tau)}\right)-\frac{\hat{\mu}}{\tilde{r}_{f}}-\frac{\partial}{\partial \xi}\left[\mu^{e x t} \hat{\mu}\right], \\
& \xi=0: \quad \hat{\mu}=1+2 y(\tau) / b-\mu_{f}^{e x t}(\tau) ; \quad \xi \leqslant-\mathrm{e}^{-y(\tau)}: \hat{\mu}=0,
\end{aligned}
$$

where $\mu^{e x t}(\xi, \tau)$ is given in (4.15) and $\tau_{s}$ denotes the time at which the overdriven regime is no more verified. The boundary conditions at $\xi=0$ and at $\xi=-\mathrm{e}^{-y(\tau)}$ are respectively the Rankine-Hugoniot relation (4.11) and (4.18).

Typically, $\xi_{s}(\tau)$ increases when $y(\tau)$ decreases, see $\S 5.1$, so that the sonic point approaches the end of the reaction $\xi_{b}(\tau)=-\mathrm{e}^{-y}$. As soon as the sonic point crosses the exit of the reaction zone, $\tau \geqslant \tau_{s}, \xi_{s}\left(\tau_{s}\right)=-\mathrm{e}^{-y\left(\tau_{s}\right)}, \mu^{e x t}\left(\xi_{s}, \tau\right)=y / b$, the propagation regime is no more overdriven; the external flow which is still solution of (2.37) in the 
burnt gas $\xi<-\mathrm{e}^{-y(\tau)}$, is influenced by the disturbances emitted from the reactive transonic flow and transported downstream (towards the weak discontinuity $\xi=\xi_{0}<0$ ) by the characteristic $\mathcal{C}_{+}$. Then, for $\tau>\tau_{s}, \xi_{s}(\tau)>-\mathrm{e}^{-y(\tau)}$, the boundary condition (4.19) is no more valid and the rarefaction wave is no more represented accurately by the analytical expression (4.15) everywhere in the burnt gas. In other words, soon after $\tau_{s}$, the rarefaction flow behind the end of the reaction $\left(\xi<-\mathrm{e}^{-y(\tau)}\right)$ is modified by the heat which is released in the region delimited by the end of the reaction and the sonic point $\left(-\mathrm{e}^{-y} \leqslant \xi \leqslant \xi_{s}\right)$. However, as in the discontinuous model in $\S 3.2$, see figures 3 and 4 , the solution (4.15) is recovered downstream until the arrival of the disturbance transported by the characteristic $\mathcal{C}_{+}$. The subsequent dynamics for $\tau \geqslant \tau_{s}$ should then be analyzed by the numerical solution of (4.10)-(4.11) using an initial condition at $\tau=\tau_{s}$ given by the solution of the eigenvalue problem (4.22)-(4.23).

\subsection{Initial condition}

In a real initiation process the trajectory of the lead shock is fully determined by the strong blast wave (inert flow) which is generated initially by a quasi-punctual and quasiinstantaneous deposit of external energy at the centre. According to the self-similar solution of Sedov (1946) - Taylor (1950b), this strong blast wave depends only on the amount of energy which is deposited, see the discussion of Liñan et al. (2012) for more details. Such an initial stage corresponds to a large Mach number which is beyond the scope of the asymptotic analysis in the limit $0<M-1 \ll 1$. The critical condition of initiation will be investigated in the present article by a parametric study of the initial conditions of the solution of (4.10)-(4.17). Three parameters of order unity in the limit $\epsilon \rightarrow 0$ are involved at $\tau=0$; the initial radius $\tilde{r}_{f i} \equiv \epsilon r_{f i} / l$, the initial thickness of the rarefaction wave $-\tilde{\xi}_{0 i} \equiv\left(r_{f i}-r_{0 i}\right) / \epsilon r_{f i}>0$, and a positive parameter $\mu_{f i}^{e x t}$. The initial propagation velocity $y_{i}=b\left(\mathcal{D}_{i}-\mathcal{D}_{o_{C J}}\right) / \epsilon a$ is related to the above-mentioned parameters through the inner structure of the detonation. Our attention will be focused on initial velocity of weakly overdriven detonations close to the CJ velocity, typically $y_{i} / b=0.15-0.5$ and on an initial extension of the rarefaction wave much larger than the detonation thickness, as in real initiation processes near criticality

$$
r_{f i}-r_{0 i} \gg l \Rightarrow\left(-\tilde{\xi}_{0 i}\right) \tilde{r}_{f i} \gg 1 \text {. }
$$

The numerical integration of (4.10)-(4.11) is initialized at $\tau=0$ by igniting the exothermal reaction in the inert flow $(4.15)$

$$
\tau=0: \quad \mu^{e x t}(\xi)=\frac{\left(1+\mu_{f i}^{e x t}\right)}{\left(-\tilde{\xi}_{0 i}\right) \tilde{r}_{f i}} \xi+\mu_{f i}^{e x t} \quad \text { with } \quad \mu_{f i}^{e x t}>0
$$

leading quickly, on a time scale shorter than unity, to the structure (4.13) of an overdriven regime sketched in figure $5, \mathcal{D}-u_{b}<a \Leftrightarrow \mu_{f i}^{e x t}\left(-\mathrm{e}^{-y_{i}}\right)-y_{i} / b>0$. For $\mu_{f i}^{e x t}$ large enough, the initial detonation velocity is larger than the CJ velocity $y_{i}>0$. As explained at the beginning of $\S 4.2$, the rarefaction flow of burnt gas (4.15)-(4.17) is not perturbed by the heat release since the regime is overdriven.

\section{Discussion of the critical dynamics}

As discussed in Clavin \& Denet (2020), the case of large activation energy leading to strongly overdriven detonations is difficult and is left for future works. Within the framework of the detonation model (4.4)-(4.9) in the limit of small heat-release, the threshold of the longitudinal instability corresponds to a critical activation energy $b=b_{c}$ 
of order unity, $b_{c}=O(1)$, for example $b_{c}=1.27$ for the distribution $\omega_{o_{C J}}(\xi)$ used in Clavin $\&$ Denet (2018). Therefore, focusing our attention on marginally stable and/or unstable detonations ( $b$ of order unity), the curvature-induced quenching which is described in the quasi-steady approximation for $b \gg 1$ in Appendix E cannot provide an accurate picture of detonation failure. However we will see that the order of magnitude of the critical radius is correctly predicted.

\subsection{Slowdown mechanism of overdriven waves. Failure and successful initiation}

Consider initial conditions for which the propagation velocity crosses the planar CJ velocity for the first time at $\tau=\tau_{t}, y\left(\tau_{t}\right)=0,-\mathrm{e}^{-y\left(\tau_{t}\right)}=-1$, with a radius small enough and a overdrive factor $\mu^{\text {ext }}\left(-1, \tau_{t}\right)>0$ large enough so that the flow at the exit of the reaction zone continues to be subsonic (relative to the lead shock) well below the planar CJ velocity, $y<0: \mu^{e x t}\left(-\mathrm{e}^{-y(\tau)}, \tau\right)-y / b>0$. According to (2.49)-(2.50), the sonic point inside the rarefaction flow of burnt gas behind an overdriven detonation moves towards the reaction zone when the acceleration $|\mathrm{d} y / \mathrm{d} \tau|$ is not too large $(\mathrm{d} y / \mathrm{d} \tau<0)$, more precisely when the decrease rate of $1+y(\tau) / b>0$ is smaller than the damping rate by the curvature

$$
-\frac{\mathrm{d}(y / b)}{\mathrm{d} \tau} \frac{1}{1+y(\tau) / b}<\frac{1}{\tilde{r}_{f}} \Leftrightarrow \frac{\mathrm{d} \xi_{s}}{\mathrm{~d} \tau}>0,
$$

see (C 16). The exit of the reaction zone $\xi=-\mathrm{e}^{-y}$ moving in the opposite direction when $y$ decreases, the overdrive factor $\mu^{e x t}\left(-\mathrm{e}^{-y(\tau)}, \tau\right)-y(\tau) / b>0$ decreases and the sonic point can catch the exit of the inner structure of the reaction wave at a later time.

The curvature term on the right-hand side of (4.10) influences the inner structure of the reaction wave and also the burnt gas flow at the exit of the reaction zone, see (4.19). These two mechanisms have an opposite effect on the dynamics of the lead shock, as shown by the following rough arguments. Considering a constant flow (subsonic/shock) at the exit of the reaction zone, the solution of (4.10)-(4.11) describes the dynamics of an overdriven wave which is isolated from the external world. In the context of direct initiation, for a velocity $y<0$ well below the CJ wave, namely for $|y|$ not small, the flow $\mu(\xi, \tau)$ in the inner structure of the combustion wave is fully out of equilibrium. Then, if the CJ wave is stable, the nonlinear relaxation towards equilibrium is expected to correspond to an increase of $y$, especially if the overdrive factor is small since the equilibrium would correspond to $y \approx 0$. Therefore, the decay of $y$ should be associated with the rarefaction-wave-induced flow at the exit of the reaction zone $\mu^{e x t}\left(-\mathrm{e}^{-y}, \tau\right)$. The corresponding response of the inner structure for adjusting the propagation velocity $y$ is thus delayed by the transit time of the characteristics $\mathcal{C}^{+} \xi=\xi_{c}\left(\tau, \tau^{\prime}\right)$ leaving the end of the reaction $\xi=-\mathrm{e}^{-y(\tau)}$ at time $\tau$ to reach the point $\xi>-\mathrm{e}^{-y}$ at a later time $\tau^{\prime}>\tau$. According to the left-hand side of the hyperbolic equation (4.10), the corresponding delay $\Delta \tau_{+}(\xi, \tau), \tau^{\prime}=\tau+\Delta \tau_{+}$, takes the form,

$$
\begin{aligned}
\xi_{s}(\tau)<-\mathrm{e}^{-y(\tau)}, \xi_{c}>-\mathrm{e}^{-y(\tau)} & , \\
\tau^{\prime} \geqslant \tau & : \quad \partial \xi_{c}\left(\tau, \tau^{\prime}\right) / \partial \tau^{\prime}=\mu\left(\xi_{c}, \tau^{\prime}\right)-y\left(\tau^{\prime}\right) / b>0, \\
\tau^{\prime}=\tau & : \quad \xi_{c}=-\mathrm{e}^{-y(\tau)}, \quad \mu=\mu^{e x t}\left(-\mathrm{e}^{-y(\tau)}, \tau\right), \\
\Delta \tau_{+}(\xi, \tau) & =\int_{-\mathrm{e}^{-y(\tau)}}^{\xi} \frac{\mathrm{d} \xi_{c}}{\mu\left(\xi_{c}, \tau+\Delta \tau_{+}\right)-y\left(\tau+\Delta \tau_{+}\right) / b}>0,
\end{aligned}
$$

reflecting the complexity of the dynamics since the delay (5.5) depends on the future of the inner structure $\mu\left(\xi, \tau^{\prime}\right), y\left(\tau^{\prime}\right), \tau^{\prime}=\tau+\Delta \tau_{+}$. Except for the integral term $Y(\tau)$ which is negligible in (4.17), the decay of the velocity $y(\tau)$ of an overdriven wave can be 
considered as slaved by the decreasing rate of the rarefaction flow $\mu^{e x t}\left(-\mathrm{e}^{-y}, \tau\right)$ but with the time delay $\Delta \tau_{+}(0, \tau)$ introduced by the characteristics $\mathcal{C}_{+}$travelling from the exit of the reaction zone to the lead shock.

This is true up to a time $\tau=\tau_{s}$ at which the sonic point catches the exit of the inner structure $\mu^{e x t}\left(-\mathrm{e}^{-y\left(\tau_{s}\right)}, \tau\right)=y\left(\tau_{s}\right) / b$ (sonic condition). When the sonic condition approaches the end of the reaction $\mu^{e x t}\left(-\mathrm{e}^{-y}\right)=y / b$ the denominator on the right-hand side of (5.5) approaches zero at the lower bound of the integral, $\partial \xi_{c}\left(\tau, \tau^{\prime}\right) /\left.\partial \tau^{\prime}\right|_{\tau^{\prime}=\tau} \rightarrow 0^{+}$, see (5.3)-(5.4), so that the time delay $\Delta \tau_{+}(0, \tau)$ which is introduced by the response of the inner structure increases strongly. Keeping in mind that the time delay becomes infinite for a CJ wave in steady state when the burnt-gas flow is uniform, $\Delta \tau_{+}(0, \tau) \rightarrow \infty$ see $\S$ C.3 of Clavin \& Denet (2020), a slowdown of the velocity decay $y(\tau)$ should occur when the sonic point $\xi_{s}(\tau)$ approaches the end of the reaction $-\mathrm{e}^{-y(\tau)}$ since the time delay of the response becomes much larger than the characteristic time of the forcing term responsible for the decay, namely the inverse of the decreasing rate of the rarefaction flow $\mu^{\text {ext }}(\xi, \tau)$. Therefore the derivative $\mathrm{d} y / \mathrm{d} \tau$ approaches 0 for $y(\tau)<0$ (local minimum below the CJ velocity), and the decay of the propagation velocity is stopped.

Consider the case for which this occurs when the propagation velocity is larger than the lower bound $y_{c}$ corresponding to the chemical kinetics quenching $y_{c}<y\left(\tau_{s}\right)<0$. Then, as soon as $\xi_{s}(\tau)+\mathrm{e}^{-y(\tau)}$ crosses zero, the domain of the inner structure corresponding to $\xi \geqslant \xi_{s}(\tau)>-\mathrm{e}^{-y(\tau)}$ becomes isolated from the rarefaction wave and thus from its damping rate. In other words the driving mechanism of the decay is switched off. As said earlier, the state of the flow inside the inner detonation-structure being out of equilibrium, a nonlinear relaxation process toward the stable CJ regime starts, so that the velocity $y$ increases after $\tau_{s}$ for ending with a success of initiation. We will come back to the slowdown mechanism in $\S 5.2$. Notice however that, for a large activation energy $b$, the success of initiation is not guarantee when the sonic point approaches the exit of the reaction zone because of a possible mechanism of curvature-induced quenching, similar to that in Appendix E. This case is not considered here and is left for future works.

In any case, a detonation failure will be produced by the chemical-kinetics quenching (4.9) if the latter occurs before the sonic condition $y\left(\tau_{s}\right) \leqslant y_{c}<0$. Another possibility is that the condition in (5.1) is not verified by the initial conditions so that the sonic point could never catch the exit of the reaction zone. This will be investigated in future works.

\subsection{Numerical results}

In order to illustrate the asymptotic analysis, preliminary numerical solutions of (4.10)(4.12) are presented with the scaling law (4.4), using the re-scaled distribution $\omega_{o_{C J}}(\xi)$, $\int_{-1}^{0} \omega_{O_{C J}}(\xi) \mathrm{d} \xi=1$, of the three-step kinetic scheme (B 16)-(B 18) in Clavin \& Denet (2018) whose instability threshold corresponds to $b=1.27$. Two values of the reduced activation energy will be considered; $b=1$ for a stable CJ wave and $b=2$ for a weakly unstable CJ wave. Typical results of a parametric study of the trajectories in the plane $y-\tilde{r}_{f}$ are presented for an initial thickness of the rarefaction wave 30 times larger than the detonation thickness, $-\tilde{\xi}_{0 i} \tilde{r}_{f i} \equiv\left(r_{f i}-r_{0 i}\right) / l=30$, by varying the initial radius $\tilde{r}_{f i} \equiv \epsilon r_{f i} / l$ in the range $0.3-3.5$ for an initial velocity $y_{i}$ in the range $0.25-0.5$.

Successful initiation and detonation failure are shown in figure 6 for a stable case, $b=1, y_{i}=0.25$ and $y_{c}=-0.5$ (chemical-kinetics quenching). A weakly unstable case for $b=2, y_{i}=0.5$ and $y_{c}=-1$ is presented in fig7. The results for such marginally unstable and stable detonations are similar, except for a nonlinear oscillation superimposed on the trajectories. For such moderate values of $b$, detonation failure is produced by the chemicalkinetics quenching. This is clearly shown by decreasing the lower bound $y_{c}$ associated with the chemical-kinetics quenching. For example, considering the case $b=1$ and $y_{c}=-0.8$, a 


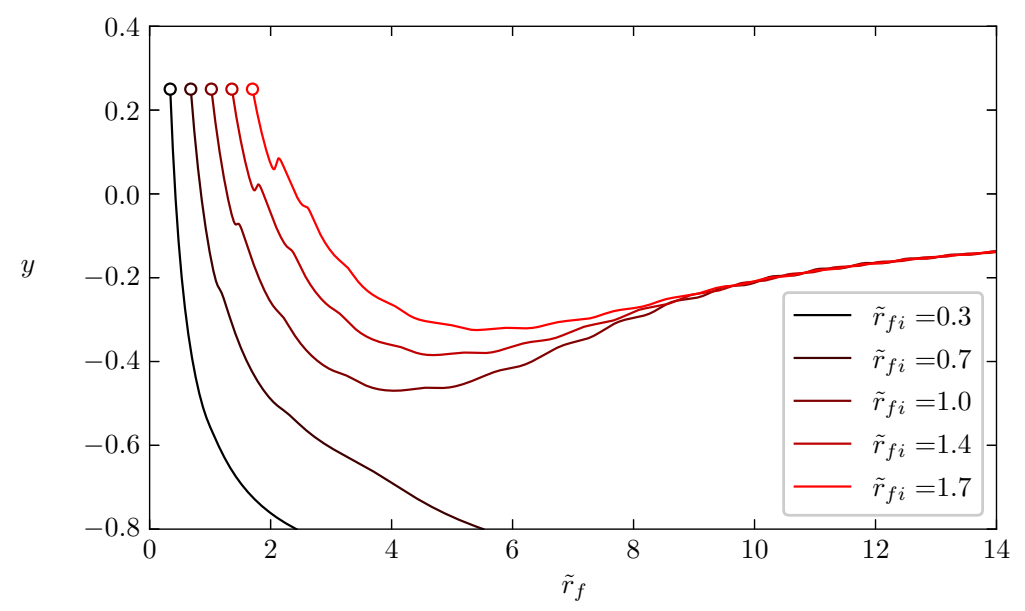

Figure 6. Examples of numerical integration of (4.10)-(4.11) for $b=1$ and $y_{c}=-0.5$. The failure occurs for a small initial radius $\tilde{r}_{f i}<0.85$ because of chemical-kinetics quenching.

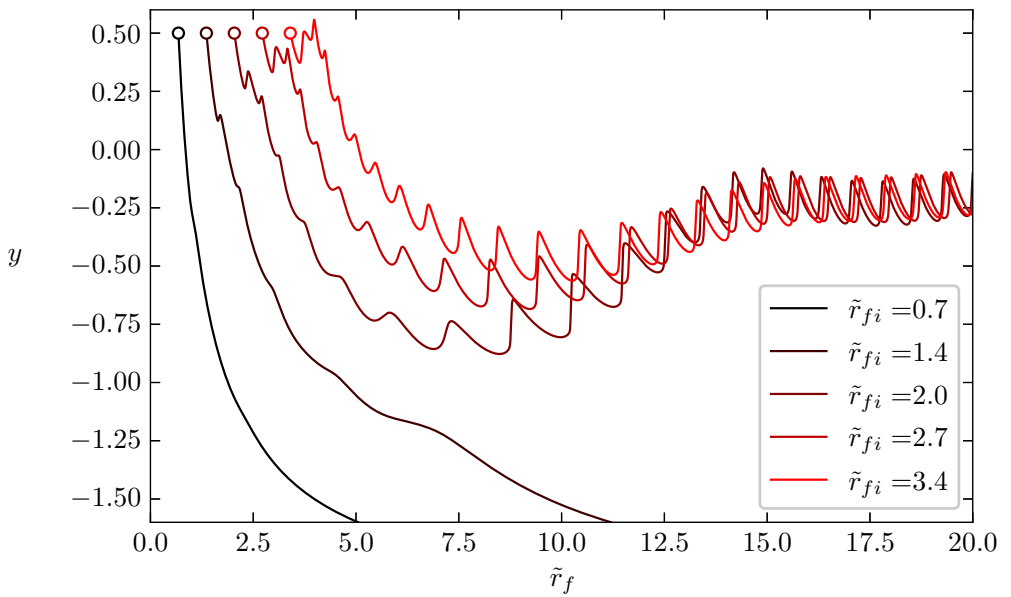

FIGURE 7. Examples of numerical integration of (4.10)-(4.11) for a weakly unstable detonation $b=2$ and $y_{c}=-1$. The failure occurs for initial radius small enough $\tilde{r}_{f i}<1.5$ because of chemical-kinetics quenching.

successful initiation is observed in figure 8 for $\tilde{r}_{f i}=0.5$ which corresponds to a detonation failure for $y_{c}=-0.5$ in figure 6 .

The slowdown mechanism, discussed in $\S 5.1$, leading to a minimum of propagation velocity well below the planar CJ velocity $y<0$, is clearly observed when the sonic point $\xi=\xi_{s}(\tau)$ approaches the end of the reaction $\xi=-\mathrm{e}^{y(\tau)}$, see figures 8 and 13 where the minimum $y \approx-0.71$ is reached at $\tau \approx 3$. During the subsequent re-acceleration of the propagation velocity $y(\tau)$ for $\tau>3$, the position of the sonic point inside the inner structure stays close to the end of the reaction, as in the solution of a steady and weakly curved CJ detonation with large activation energy, but, here the flow in the inner-detonation structure is out of equilibrium, as already mentioned. Notice that the minimum of propagation velocity $y(\tau)$ occurs just before the sonic point catches the 

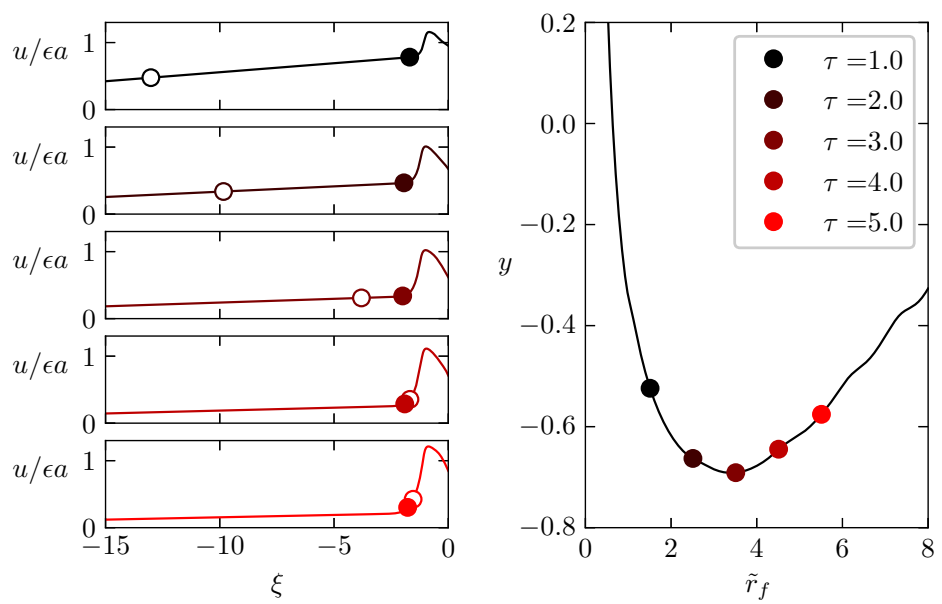

FiguRE 8. Numerical integration of (4.10)-(4.11) for a stable detonation $b=1$ for $y_{c}=-0.8$ and $\tilde{r}_{f i}=0.5$, showing a successful initiation. The profiles of the reduced flow velocity $u(\xi, \tau) / \epsilon a$ are plotted on the left at different times. The trajectory $y\left(\tilde{r}_{f}\right)$ is plotted on the right. The full points denote the exit of the reaction zone and the open circles on the left are the sonic points (relative to the lead shock).

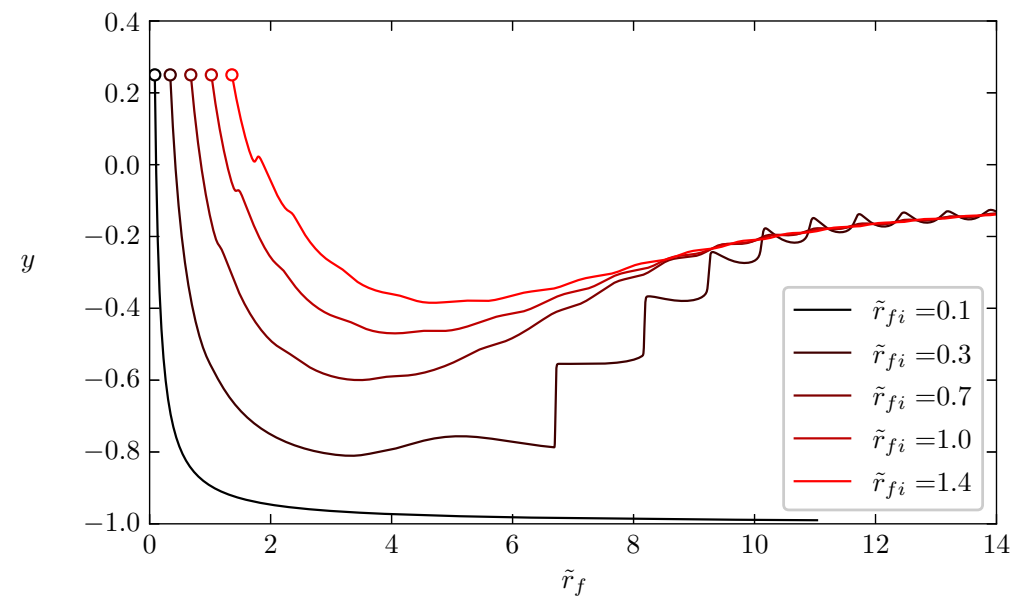

Figure 9. Numerical integration of (4.10)-(4.11) for stable detonation, $b=1$ and $y_{c}=-0.8$. The trajectory corresponding to $\tilde{r}_{f i}=0.1$ describes a failure of initiation $y(\tau) \rightarrow-1$ and that corresponding to $\tilde{r}_{f i}=0.3$ is a successful initiation with jumps during the increase of the detonation velocity resulting from the formation of shock waves in the induction zone, as explained in the text.

exit of the inner structure of the reaction wave. This means that the external damping rate is balanced by the internal mechanism of re-acceleration for a small overdrive factor $\mu^{e x t}\left(-\mathrm{e}^{-y(\tau)}, \tau\right)-y(\tau) / b>0$, a little bit before $\tau_{s}$ for which the sonic condition is obtained $\mu^{e x t}\left(-\mathrm{e}^{-y\left(\tau_{s}\right)}, \tau\right)-y\left(\tau_{s}\right) / b=0$. Notice also that the minimum of $y(\tau)$ corresponds to a non-dimensional radius $\tilde{r}_{f} / b$ of order unity as for the critical radius of the $\mathrm{C}$-shaped curve obtained by the quasi-steady state approximation, see Appendix E. 


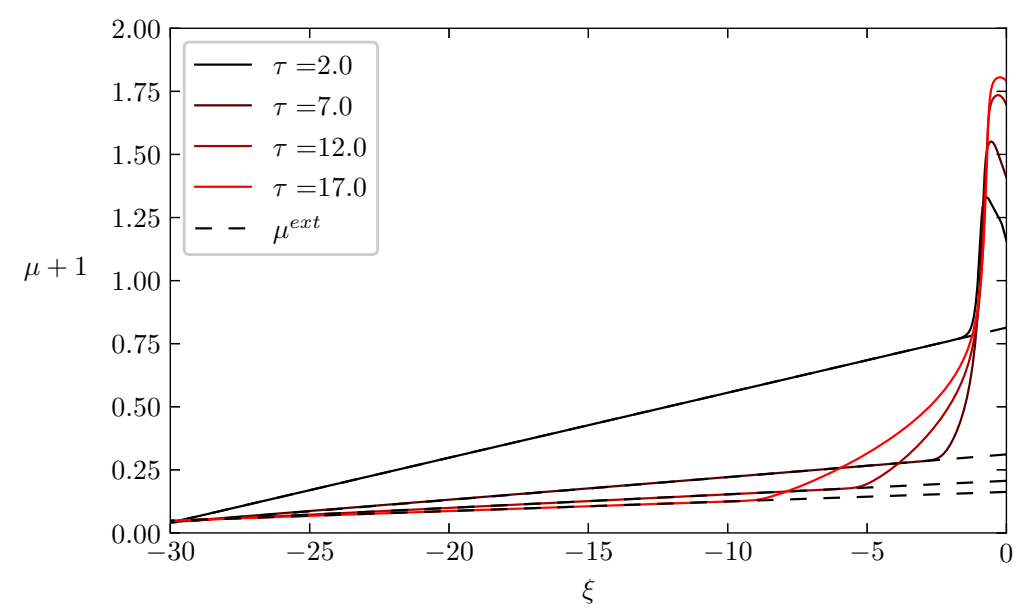

Figure 10. Comparison of the numerical solution with the theoretical expression (4.15)-(4.17) of the rarefaction wave $\mu^{e x t}(\xi, \tau)$ for a successful initiation.

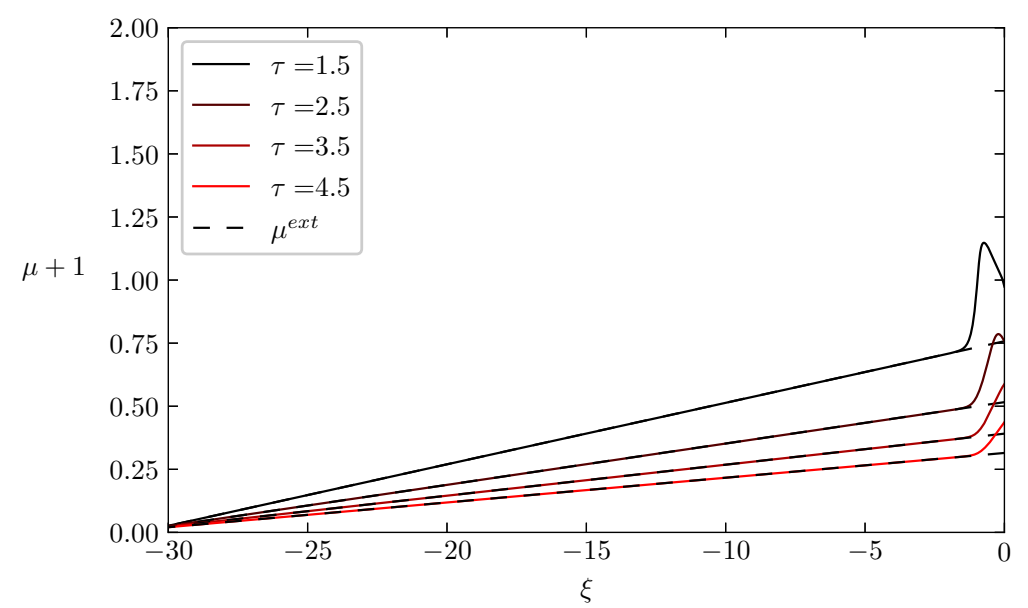

FIGURE 11. Comparison of the numerical solution with the theoretical expression (4.15)-(4.17) of the rarefaction wave $\mu^{e x t}(\xi, \tau)$ for a detonation failure.

Inside the induction zone where the heat release is negligible, $\omega \approx 0$, the curvature term on the right-hand side of (4.10) makes the instantaneous distribution of the flow decreasing when approaching the lead shock, $\partial \mu / \partial \xi<0$. Then, the distribution $\mu(\xi, \tau)$ presents a maximum inside the inner structure of the reaction wave, clearly shown on figures 8 left and 10. The corresponding peak of pressure was observed in direct numerical simulations and was considered by $\mathrm{Ng}$ \& Lee (2003) to be the driving mechanism of the re-acceleration of the wave leading to the detonation initiation in the critical regime. The flow velocity decreasing in space in the direction of propagation, formation of shock waves is possible inside the induction zone. This is observed on the numerical solutions of (4.10)(4.12) during the increase of the detonation velocity for trajectories whose minimum of $y$ is just above the lower bound (chemical-kinetics quenching) when the latter is small, 


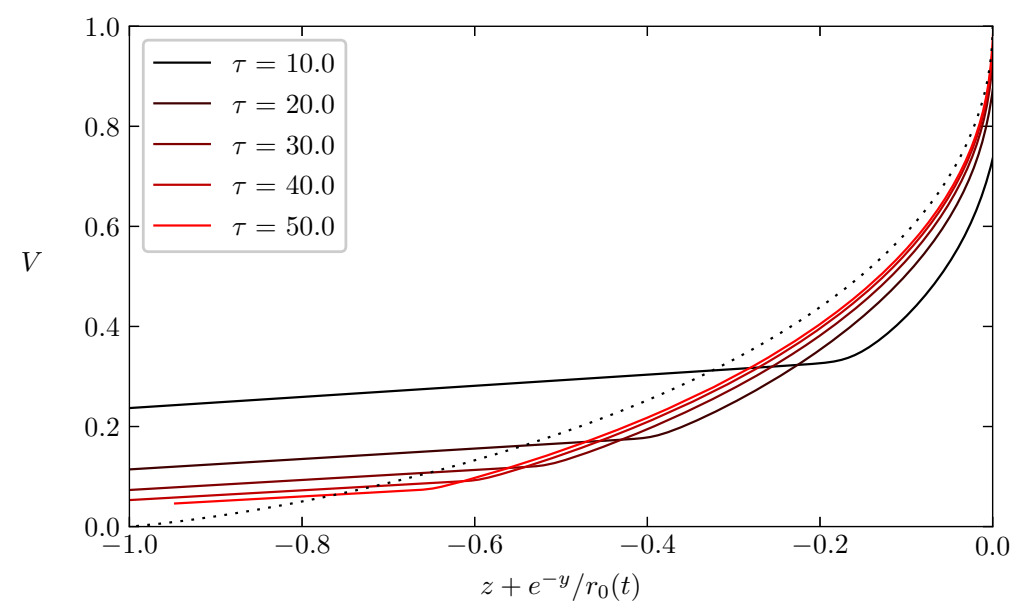

Figure 12. Numerical solutions at different times compared to the self-similar solution of (2.23)-(2.24) (dotted line) representing the self-similar rarefaction flow behind the front of a spherical CJ detonation considered as a discontinuity for small heat release $\left(\mathcal{D}_{o_{C J}}-a\right) / a \approx \epsilon \ll 1$. The reduced flow $\mathrm{v}=\mathrm{u}(r, t) / \epsilon a$ is plotted versus the reduced distance from the front $z=\left(r-r_{f}(t)\right) / \epsilon r_{0}(t)$ with $r_{f}(t)=\mathcal{D}_{o_{C I}} t+r_{f i}, r_{0}(t)=a t+r_{0 i}$ and, according to (2.26), $r_{f i}=(1+\epsilon) r_{0 i}$. Because of the finite thickness of the reaction zone $\mathrm{e}^{-y}$ the origin of space variable has been shifted in the figure.

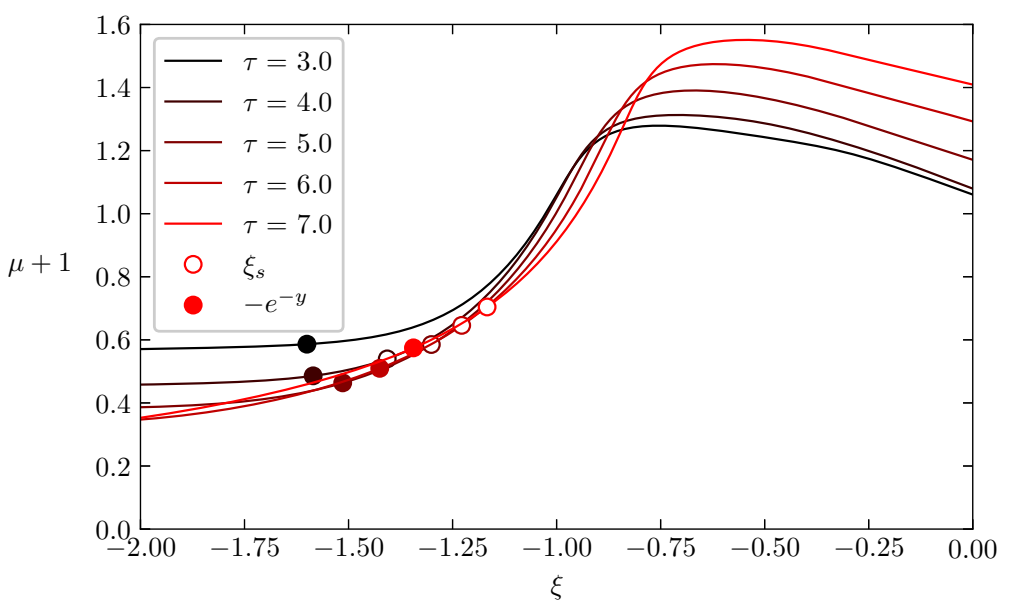

FIGURE 13. Zoom of the velocity profiles for the same parameters as in figure 10 for later time. The sonic point is out of the picture at $\tau=3$

$0<(\mathcal{D}-a) / \epsilon a \equiv 1+y / b \approx 0^{+}, y+b \ll b$. This occurs for initial radius small enough, see for example the trajectory corresponding to $\tilde{r}_{f i}=0.3$ in figure 9 . The physical relevance of a detonation velocity becoming so close to $y=-b$ is questionable since it corresponds to a crossover temperature for the chemical-kinetics quenching close to the initial temperature, since typically in real detonations the chemical-kinetics quenching corresponds to $y_{c}=-b / 2$, see the paragraph above (4.9). The phenomenon of shock formation during the re-acceleration was also observed in the direct numerical simulations 
of $\mathrm{Ng} \&$ Lee (2003) for a one-step kinetic rate law in a planar geometry. This question deserves more work.

Comparison between the numerical solution and the asymptotic analysis shows a very good agreement, confirming the validity and relevance of both numerical method and analytical solution. Solving numerically (4.10)-(4.12) for an initial condition (4.25), down to the radius of the core of stagnant gas $\xi=\xi_{0}(\tau)$ where $u=0$, a straight part of the numerical solution is observed. The latter fits with an excellent accuracy the theoretical expression $\mu^{e x t}(\xi, \tau)$ in (4.15)-(4.17) for either successful initiation or failure, see figures 10 and 11. Moreover, the theoretical result (4.17) with $Y=0$ fits also with a good accuracy the numerical result. This could be quite helpful for further theoretical analyses.

Moreover the numerical solution of successful initiation clarifies the question raised long ago by Taylor (1950a) concerning the link between the self-similar solution and the trajectory "detonation velocity versus radius" for a successful initiation when the inner structure of the reaction wave is taken into account. A first indication is given by the instantaneous profiles of the burnt-gas flow behind the reaction zone in figure 10 for $\tau \geqslant 7$ showing similitudes with the discontinuous model in figure 4 . The selfsimilar profile of figure 1 is recovered by the numerical result in the long-time limit $\tilde{\tau} \equiv \tau / \tilde{r}_{f i} \gg-\tilde{\xi}_{0 i}$ when the reduced flow $\mu(\xi, \tau)+1$ is plotted versus the self-similar variable $(2.25)\left[r-r_{f}(t)\right] /\left[r_{f}(t)-r_{0}(t)\right] \equiv \tilde{\xi} /\left(-\tilde{\xi}_{0}\right)$ with $\tilde{\xi}_{0}=-\tilde{\tau}+\tilde{\xi}_{0 i}$. This is shown in figure 12. According to the zoom in figure 13, the slope $\partial \mu /\left.\partial \xi\right|_{\xi=-\mathrm{e}^{-y}}$, which is initially small (at the scale of the detonation thickness $\xi=O(1)$ ) in the overdriven regimes, increases when the sonic point gets into the inner structure, so that the flow gradient becomes infinite at the scale of the thickness of the rarefaction wave which increases linearly with the time.

\section{Conclusion and perspectives}

The critical dynamics of the direct initiation of detonation is studied in the limit of small heat release reducing the problem to solve a single hyperbolic equation. This limit emphasizes the two-time-scale nature of the problem which also characterizes real detonations when the condition at the exit of the reaction zone is nearly sonic. The simplification comes from a flow Mach number differing from unity by a small amount everywhere. In real detonations near the CJ regime, this is true at the end of the reaction zone, but not close to the lead shock. Concerning the trajectories of the lead shock in direct initiation, the limit of small heat release introduces differences that are mainly quantitative, except for the overshoot during the re-acceleration phase which is sometimes observed in direct numerical simulations. Useful results of the asymptotic limit are the analytical expressions of the rarefaction flow for both discontinuous model of detonation and combustion waves whose inner structure is unsteady. Amazingly, these asymptotic solutions present the same characteristic properties as those of the flow in the opposite limit of a large Mach number of propagation. This confirms the relevance of the limit of small heat release for improving our understanding of the detonation dynamics.

The evolution of the sonic point (sonic condition relatively to the lead shock) inside the rarefaction wave is then found to be the key element of the overall dynamics. Due to the increase of the time delay by the response of the detonation inner-structure to the burnt-gas flow, a slowdown mechanism is identified when the sonic point, located initially in the burnt gas away from the reaction wave (overdriven regimes), approaches the exit of the reaction zone. This mechanism explains the behavior of the trajectories "propagation velocity versus radius" observed near criticality in direct numerical simulations of successful initiation. The detonation velocity decreases well below the CJ velocity like for 
a failure and reaches a minimum associated with the onset of the sonic condition. This deceleration phase is followed by a re-acceleration back to the CJ regime corresponding to an isolated combustion wave whose inner structure is out of equilibrium. During this nonlinear relaxation, the sonic point stays inside the inner structure of the combustion wave, close to the end of the reaction zone. Failure is produced if the detonation velocity decreases so much that the chemical-kinetics quenching occurs in overdriven regimes when the sonic point is still in the burnt-gas behind the exit of the reaction zone. This depends on the radius at which the velocity of the overdriven detonation crosses the CJ velocity for the first time; the smaller the radius, the stronger the damping rate and the detonation is more likely. Another outcome of the asymptotic analysis is to indicate how the self-similar CJ solution for the rarefaction wave is reached in the long time limit, showing a behavior similar to the discontinuous model.

Due to the difficulty pointed out in Clavin \& Denet (2020) for strongly unstable detonations in the limit of small heat release using the scaling law (4.4), the attention was limited in this article to a reduced activation energy of order unity $b=O(1)$. This prevents us to see whether or not the curvature-induced quenching which is predicted without multiple-step chemistry (no crossover temperature) by a quasi-steady approximation for large $b$, can also occur in the unsteady regime after the sonic point has caught the end of the reaction. Notice however that the radius at the minimum of velocity is of same order of magnitude as the quasi-steady critical radius. Future works will be devoted to this question. This should be performed with an unsteady reaction rate different from the scaling law (4.4). More generally, improvements of the asymptotic analysis in the limit of small heat release will be sought to provide a better quantitative accuracy with real detonations. Another point which deserves more investigation is the re-ignition by shock waves observed when the minimum velocity is very low.

In this paper the analysis of the critical dynamics is limited to a spherical geometry. The result of plane or cylindrical detonations cannot be conjectured by dimensional arguments, the analysis has to be reconsidered from the beginning. The dynamics of the sonic point inside the rarefaction wave is expected to also play an essential role.

Declaration of interests. The authors report no conflict of interest

\section{Acknowledgements}

Long-standing enlightening discussions with Amable Liñan have motivated this work. Partial financial support of Agence National de la Recherche (contract ANR-18-CE050030) is acknowledged.

\section{Appendix A. Conditions in the burnt gas behind the detonation front}

The conditions behind a detonation treated as a discontinuity is obtained by the conservation of mass, momentum and energy across a planar detonation in quasi-steady state. They can be put in the form, see Clavin \& Searby (2016),

$$
\begin{aligned}
& M \geqslant M_{o_{C J}}>1: 1-\frac{\rho_{u}}{\rho_{b}}=\frac{1}{\gamma+1} \frac{\left(M^{2}-1\right)}{M^{2}}\left[1+\sqrt{1-\left(\frac{M_{o_{C J}}-M_{o_{C J}}^{-1}}{M-M^{-1}}\right)^{2}}\right] \\
& \frac{p_{b}}{p_{u}}-1=\gamma M^{2}\left(1-\frac{\rho_{u}}{\rho_{b}}\right), \quad \frac{u_{b}}{a_{u}}=\left(1-\frac{\rho_{u}}{\rho_{b}}\right) M, \quad M_{o_{C J}}-M_{o_{C J}}^{-1}=2 \sqrt{Q}
\end{aligned}
$$


where the subscripts $u$ and $b$ denote respectively the fresh mixture and the burnt gas, $Q \propto q_{m} / c_{p} T_{u}$ is the reduced heat release and $M \equiv \mathcal{D} / a_{u}$ is the propagation Mach number. The square root is zero for the CJ regime corresponding to the sonic condition in the burnt gas $M=M_{o_{C J}}: u_{b o_{C J}}=\mathcal{D}_{o_{C J}}-a_{b o_{C J}}$ with $a_{b o_{C J}}=\sqrt{\gamma p_{b o_{C J}} / \rho_{b o_{C J}}}$. According to the last equation in (A 2), the CJ Mach number is close to unity in the limit of small heat release, $0<\left(M_{o_{C J}}-1\right) \ll 1$. For weakly overdriven regimes $0<\left(M-M_{o_{C J}}\right) / M_{o_{C J}} \ll 1$ the square root in (A 1$)$ is small so that the propagation Mach number is also close to unity $0<(M-1) \ll 1$ for small heat release and the flow velocity in the laboratory frame is much smaller than the sound speed $u_{b} / a_{u}=O(M-1)$. However the rarefaction wave is not a spherical acoustic wave even though it is a linear function of the radius behind a weakly overdriven detonation $M>M_{o C J}$, as shown $\S 2.3$, while the rarefaction wave behind a spherical CJ detonation investigated in $\S 2.2$ is quite different.

The Rankine-Hugoniot conditions at the Neumann state of an inert shock corresponds to (A 1)-(A 2) when the square root on the right-hand side of (A 1) is set equal to unity,

$$
\begin{gathered}
Q=0: \quad 1-\frac{\rho_{u}}{\rho_{N}}=\frac{2}{\gamma+1} \frac{\left(M^{2}-1\right)}{M^{2}}, \quad \frac{p_{N}}{p_{u}}-1=\gamma M^{2}\left(1-\frac{\rho_{u}}{\rho_{N}}\right) \\
\frac{u_{N}}{a_{u}}=M\left(1-\frac{\rho_{u}}{\rho_{N}}\right), \quad \frac{T_{N}}{T_{u}}=\frac{1}{M^{2}}\left[1+\frac{2 \gamma}{\gamma+1}\left(M^{2}-1\right)\right]\left[1+\frac{\gamma-1}{\gamma+1}\left(M^{2}-1\right)\right] \\
M-1 \ll 1: \quad \frac{u_{N}}{a_{u}} \approx \frac{4}{\gamma+1}(M-1), \quad \frac{T_{N}}{T_{u}}-1 \approx \frac{4(\gamma-1)}{\gamma+1}(M-1) \\
\frac{p_{N}}{p_{u}}-1 \approx \frac{4 \gamma}{\gamma+1}(M-1)
\end{gathered}
$$

where the subscript $N$ denotes the Neumann state.

\section{Appendix B. Linear solution}

A linear solution of the small flow behind a spherical detonation, treated as a discontinuity in the limit of small heat release $\left(0<M_{o_{C J}}-1 \ll 1\right)$, is performed here. By comparison with the development in $\S 2$, the purpose is to stress the nonlinear character of the rarefaction wave when approaching the CJ regime.

\section{B.1. Spherical acoustic wave}

Let's first briefly recall the acoustic waves in a spherical geometry. The linear version of the isentropic Euler equations yields

$$
\frac{\partial\left(\rho-\bar{\rho}_{b}\right) / \bar{\rho}_{b}}{\partial t}+\nabla . \mathbf{u}=0, \quad \frac{\partial \mathbf{u}}{\partial t}=-\frac{a^{2}}{\bar{\rho}_{b}} \nabla \rho,
$$

where $\left(\rho-\bar{\rho}_{b}\right) / \bar{\rho}_{b} \ll 1$ with $\bar{\rho}_{b}$ and $a=a_{b} \approx a_{u}$ constant. Introducing the potential $\varphi$, equations (B 1) yield

$$
\frac{\left(\rho-\bar{\rho}_{b}\right)}{\bar{\rho}_{b}}=\frac{\partial \varphi}{\partial t}, \quad \mathbf{u}=-a^{2} \nabla \varphi, \quad \frac{\partial^{2} \varphi}{\partial t^{2}}-a^{2} \Delta \varphi=0
$$

where $\Delta \varphi=\frac{1}{r^{2}} \frac{\partial}{\partial r}\left(r^{2} \frac{\partial \varphi}{\partial r}\right)$ in spherical geometry. Introducing the function $f(r, t)$, and looking for the solution in the form $\varphi=f / r$,

$$
\frac{\partial^{2} f}{\partial t^{2}}-a^{2} \frac{\partial^{2} f}{\partial r^{2}}=0
$$


the flow expressed in terms of the non-dimensional the transit time $\mathrm{r} \equiv r / a$ is

$$
\begin{aligned}
\varphi & =\frac{f_{1}(t-\mathrm{r})+f_{2}(t+\mathrm{r})}{\mathrm{r}} \Rightarrow \frac{\left(\rho-\bar{\rho}_{b}\right)}{\bar{\rho}_{b}}=\frac{f_{1}^{\prime}(t-\mathrm{r})+f_{2}^{\prime}(t+\mathrm{r})}{\mathrm{r}} \\
v(\mathrm{r}, t) & \equiv \frac{u(\mathrm{r}, t)}{a}=\frac{f_{1}^{\prime}(t-\mathrm{r})-f_{2}^{\prime}(t+\mathrm{r})}{\mathrm{r}}+\frac{f_{1}(t-\mathrm{r})+f_{2}(t+\mathrm{r})}{\mathrm{r}^{2}},
\end{aligned}
$$

where $f_{1}^{\prime}(\eta)$ and $f_{2}^{\prime}(\eta)$ denote the derivative of $f_{1}(\eta)$ and $f_{2}(\eta), f_{1}^{\prime}(\eta) \equiv \mathrm{d} f_{1}(\eta) / \mathrm{d} \eta$, $f_{2}^{\prime}(\eta) \equiv \mathrm{d} f_{2}(\eta) / \mathrm{d} \eta$, the unknown functions $f_{1}(\eta)$ and $f_{2}(\eta)$ being determined by the boundary conditions.

Consider the flow behind a supersonic front $r=r_{f}(t)$ propagating with the velocity $\mathcal{D}(t)=\mathrm{d} r_{f} / \mathrm{d} t, M(t)=\mathcal{D}(t) / a>1$. Denoting $u_{b}(t)$ and $\rho_{b}(t)$ the flow velocity and the density on the lead front and introducing the notations $\mathrm{r}_{f}(t)=r_{f}(t) / a, \mathrm{dr}_{f} / \mathrm{d} t=M(t)$

$$
\begin{array}{lll}
F_{1}(t) \equiv f_{1}\left(t-\mathrm{r}_{f}(t)\right), & \dot{F}_{1}(t) \equiv \mathrm{d} F_{1} / \mathrm{d} t=[1-M(t)] f_{1}^{\prime}\left(t-\mathrm{r}_{f}(t)\right), \\
F_{2}(t) \equiv f_{2}\left(t+\mathrm{r}_{f}(t)\right), & \dot{F}_{2}(t) \equiv \mathrm{d} F_{2} / \mathrm{d} t=[1+M(t)] f_{2}^{\prime}\left(t+\mathrm{r}_{f}(t)\right),
\end{array}
$$

the boundary conditions at the front take the form

$$
\begin{aligned}
\frac{1}{\mathrm{r}_{f}(t)}\left[\frac{\dot{F}_{1}(t)}{1-M(t)}+\frac{\dot{F}_{2}(t)}{1+M(t)}\right] & =\frac{\delta \rho_{b}(t)}{\bar{\rho}_{b}} \equiv \frac{\left(\rho_{b}(t)-\bar{\rho}_{b}\right)}{\bar{\rho}_{b}} \\
\frac{1}{\mathrm{r}_{f}(t)}\left[\frac{\dot{F}_{1}(t)}{1-M(t)}-\frac{\dot{F}_{2}(t)}{1+M(t)}\right]+\frac{F_{1}(t)+F_{2}(t)}{\mathrm{r}_{f}^{2}(t)} & =\frac{u_{b}(t)}{a}
\end{aligned}
$$

where $\rho_{b}$ and $u_{b} / a$ are expressed in terms of the propagation Mach number $M(t)$ through (A 1)-(A 2). The radius at which the flow vanishes $u\left(r_{0}(t), t\right)=0, \mathrm{~d} u /\left.\mathrm{d} r\right|_{r-r_{0}=0^{+}}>0$ should be a weak discontinuity,

$$
r=r_{0}(t): u(r, t)=0, \quad r-r_{0}(t)=0^{+}: \mathrm{d} r_{0} / \mathrm{d} t=a<\mathcal{D} .
$$

\section{B.2. Solution}

We look for the linear solution for the flow behind a CJ regime in a spherical geometry treated as a discontinuity propagating with the constant velocity of the planar wave in steady state $\mathcal{D}=\mathcal{D}_{o C J}\left(\right.$ constant Mach number $\left.M=M_{o_{C J}} \equiv \mathcal{D}_{o_{C J}} / a>1\right)$

$$
r_{f o_{C J}}(t)=\mathcal{D}_{o_{C J}} t+r_{f o i}, \quad \mathrm{r}_{f o_{C J}}(t)=M_{o_{C J}} t+\mathrm{r}_{f o i}, \quad \mathrm{r}_{f o i}=\text { cst. }
$$

According to (A 1) and (A 2) for the square root $=0$, the right-hand side of (B 8) and (B 9) are constant,

$$
1-\frac{\rho_{u}}{\rho_{b o_{C J}}}=\frac{1}{M_{o_{C J}}} \frac{u_{b o_{C J}}}{a}, \quad \frac{u_{b o_{C J}}}{a}=\frac{1}{\gamma+1} \frac{\left(M_{o_{C J}}^{2}-1\right)}{M_{o_{C J}}} .
$$

Taking $\bar{\rho}_{b}=\rho_{b o_{C J}}$, the right-hand side of (B 8) is zero,

$$
r=r_{f o_{C J}}(t): \quad u_{b}=u_{b o_{C J}}, \quad \rho_{b}-\bar{\rho}=\rho_{b}-\rho_{b o_{C J}}=0 .
$$

For small heat release $\epsilon \equiv\left(M_{o_{C J}}-1\right) \ll 1, u_{b_{C J}} / a \ll 1 \Rightarrow u / a \ll 1$, the linear solution (B 4)-(B 5) with (B 8)-(B 9) satisfying the boundary conditions (B 12)-(B 13) is

$$
\begin{aligned}
r_{0}(t) \leqslant r \leqslant r_{f o_{C} J}: \frac{u(\mathrm{r}, t)}{u_{b o_{C} J}} & =\left[1-\frac{1}{M_{o_{C J}}^{2}-1}\left(\frac{r_{f o_{C J}}^{2}(t)}{r^{2}}-1\right)\right], r \leqslant r_{0}(t): u=0, \\
1-\frac{\rho(\mathrm{r}, t)}{\rho_{b o_{C J}}} & =\frac{u_{b o_{C J}}}{a} \frac{2 M_{o_{C J}}}{M_{o_{C J}}^{2}-1}\left(\frac{r_{f o_{C J}}(t)}{r}-1\right), r \leqslant r_{0}(t): \rho=\rho_{u}(\mathrm{~B}
\end{aligned}
$$


where, according to (A 1$), u_{b_{C J}} / a=\left(M_{o_{C J}}^{2}-1\right) M_{o_{C J}}^{-1} /(\gamma+1)$. The solution also satisfies the downstream condition (B 10) of a weak discontinuity provided $r_{0}(t) \equiv a t+r_{f o i} / M_{o_{C J}}$

$$
r_{0}(t) \equiv a t+r_{f o i} / M_{o_{C J}} \Rightarrow r_{f o i}(t) / r_{0}(t)=M_{o_{C J}} \Rightarrow r=r_{0}(t): u=0, \rho=\rho_{u}
$$

the last relation being valid to leading order in the limit $\epsilon \rightarrow 0,(\gamma-1) \rightarrow 0$. Therefore the thickness of the rarefaction wave $r_{f o_{C J}}-r_{0}$ increases also linearly with the time

$$
\frac{r_{f o_{C J}}(t)-r_{0}(t)}{r_{0}(t)}=M_{o_{C J}}-1, \quad \frac{1}{a} \frac{\mathrm{d}}{\mathrm{d} t}\left(r_{f o_{C J}}-r_{0}\right)=M_{o_{C J}}-1,
$$

The calculation proceeds as follows. Using (B 10) and (B 13) integration of (B 8) yields

$$
\frac{F_{1}(t)}{1-M}+\frac{F_{2}(t)}{1+M}=A^{\prime} \Rightarrow F_{2}=-\frac{1+M}{1-M} F_{1}+(1+M) A^{\prime}
$$

where $A^{\prime}$ is a constant. In order to save the notation the subscript $o_{C J}$ has been omitted in $M_{O_{C J}}$ which is replaced by $M$. Adding (B 8) and (B 9) yields a differential equation for $F_{1}(t)$ when $F_{2}(t)$ is eliminated by using (B 17)

$$
\left(t+\mathrm{r}_{f o i} / M\right) \dot{F}_{1}-F_{1}=-A+\left(t+\mathrm{r}_{f o i} / M\right)^{2}\left(u_{b} / a\right)(1-M) M / 2
$$

where $A=\left(1-M^{2}\right) A^{\prime} /(2 M)$ is constant. Equation (B 18) is easily integrated

$$
F_{1}(t)=A+\left(t+\mathrm{r}_{f i} / M\right) B+\left(t+\mathrm{r}_{f i} / M\right)^{2}\left(u_{b} / a\right)(1-M) M / 2,
$$

where $B$ is another constant and $F_{2}(t)$ is obtained from (B 17). According to (B 6)(B 7), $f_{1}(t-\mathrm{r})$ and $f_{2}(t+\mathrm{r})$ are obtained from $F_{1}(t)$ and $F_{2}(t)$ by the substitution $t \rightarrow\left[(t-\mathrm{r})+\mathrm{r}_{f i}\right] /(1-M)$ and $t \rightarrow\left[(t+\mathrm{r})+\mathrm{r}_{f i}\right] /(1+M)$ respectively,

$$
\begin{aligned}
& f_{1}(t-\mathrm{r})=A+\frac{B}{1-M}\left[(t-\mathrm{r})+\frac{\mathrm{r}_{f i}}{M}\right]+\left(u_{b} / a\right) \frac{M / 2}{1-M}\left[(t-\mathrm{r})+\frac{\mathrm{r}_{f i}}{M}\right]^{2} \\
& f_{2}(t+\mathrm{r})=-A-\frac{B}{1-M}\left[(t+\mathrm{r})+\frac{\mathrm{r}_{f i}}{M}\right]-\left(u_{b} / a\right) \frac{M / 2}{1+M}\left[(t+\mathrm{r})+\frac{\mathrm{r}_{f i}}{M}\right]^{2} .
\end{aligned}
$$

The constants $A$ and $B$ disappear from (B 5) leading to (B 14).

Because of the sonic condition at the front this linear solution is not self-consistent since the nonlinear terms are essential near the sonic condition as discussed now.

\section{B.3. Inconsistency of the linear approximation behind a CJ detonation}

The flow (B 14) is effectively small for $r_{0}(t) \leqslant r \leqslant r_{f o_{C J}}(t)$ when the heat release is small

$$
\epsilon^{2} \equiv\left[q_{m} / c_{p} T_{u}\right](\gamma+1) / 2 \ll 1, \quad\left(M_{o_{C J}}-1\right) \approx \epsilon, \quad \mathcal{D}_{o_{C J}} \approx(1+\epsilon) a
$$

because the flow increases from 0 to its value at the leading edge of the rarefaction wave $u_{b o_{C J}}$ which is, according to (B 12), smaller than the sound speed $u_{b o_{C J}} / a \approx \epsilon$

$$
\begin{aligned}
\frac{r_{f o_{C J}}(t)-r_{0}(t)}{r_{0}(t)} & =\epsilon, \quad r_{f o_{C J}}(t)=(1+\epsilon) r_{0}(t), \quad \frac{r_{f o_{C J}}(t)}{r}-1=O(\epsilon), \\
\frac{u_{o_{C J}}(r, t)}{a} & =\frac{2}{\gamma+1}\left[\epsilon-\left(\frac{r_{f o_{C J}}(t)}{r}-1\right)\right]+O\left(\epsilon^{2}\right), \\
\frac{1}{a} \frac{\partial u_{o_{C J}}}{\partial t} & =-\frac{2}{\gamma+1}(1+\epsilon) \frac{a}{r} \approx(1+\epsilon) \frac{a}{r}, \quad \frac{1}{a} \frac{\partial u_{o_{C J}}}{\partial r} \approx \frac{r_{f o_{C J}}(t)}{r^{2}} .
\end{aligned}
$$

Inside the rarefaction wave, $r_{0}(t) \leqslant r \leqslant r_{f o_{C J}}(t), r / r_{0}(t)-1=O(\epsilon)$, to leading order in the limit $\epsilon \ll 1$, the velocity profile is linear since, according to (B 23)-(B 16)

$$
r_{0}(t) \leqslant r \leqslant r_{f o_{C J}}(t): \quad \frac{(\gamma+1)}{2} \frac{u_{o_{C J}}(r, t)}{a}=\frac{(1+\epsilon)\left[r-r_{0}(t)\right]}{r} \in[0, \epsilon],
$$


neglecting terms of order $\epsilon^{2}$, one has

$$
r_{0}(t) \leqslant r \leqslant r_{f_{o_{C}}}(t): \quad \frac{(\gamma+1)}{2} \frac{u_{o_{C J}}(r, t)}{a} \approx \frac{r-r_{0}(t)}{r_{0}(t)} \in[0, \epsilon] .
$$

The physical interpretation is simple, the numerator on the right-hand side of (B 27) describes a propagation at the speed of sound while the denominator is the geometrical damping in a spherical geometry. The linear solution (B 27) is different from the selfsimilar solution in figure 1. It is also different from the rarefaction wave (2.51)-(2.52), even though they are both linear in space. The flow (B 27) is effectively solution of the linear version of (2.9) for $u \ll a$ and $r_{f}(t)-r_{0}(t)=\epsilon r_{0}(t), r \approx r_{0}(t)$,

$$
\frac{\partial u}{\partial t}+a \frac{\partial u}{\partial r}=-a \frac{u}{r_{0}(t)}, \quad r_{0}(t)=a t+r_{0 i},
$$

as verified by the general solution of (B 28) for the initial condition $u(r, t=0)=u_{i}(r)$, $u=u_{i}(r-a t) r_{0 i} / r_{0}(t)$, leading to (B 27) if the initial flow increases linearly in space $u_{i}(r)=a\left(r-r_{0 i}\right) / r_{0 i}$. The inconsistency of the linear solution (B 27) is pointed out by computing the nonlinear term $u \partial u / \partial r=a^{2}\left(r-r_{0}\right) / r_{0}^{2}=a u / r_{0}$ which has the same order of magnitude as the right-hand side. This illustrates that the nonlinear term $u \partial u / \partial r$ plays an essential role in the transonic flow relative to the front.

\section{Appendix C. Details of calculation}

C.1. Equation (2.31)

Using the notations (2.28), equation (2.10) takes the form

$$
\begin{aligned}
& {\left[\frac{\epsilon}{t_{r}} \frac{\partial}{\partial \tau}+\frac{1}{t_{r}}\left(\frac{u}{a}+1-\frac{\mathcal{D}}{a}\right) \frac{\partial}{\partial \xi}\right] \mu=-a \frac{(1+\mu)}{r},} \\
& \mathcal{D}_{o_{C J}} / a=1+\epsilon \Rightarrow\left[\epsilon \frac{\partial}{\partial \tau}+\left(\frac{u}{a}-\epsilon-\frac{\left(\mathcal{D}-\mathcal{D}_{o_{C J}}\right)}{a}\right) \frac{\partial}{\partial \xi}\right] \mu=-\frac{l}{r}(1+\mu), \\
& {\left[\epsilon \frac{\partial}{\partial \tau}+\left(\epsilon \mu-\frac{\left(\mathcal{D}-\mathcal{D}_{o_{C J}}\right)}{a}\right) \frac{\partial}{\partial \xi}\right] \mu=-\frac{l}{r}(1+\mu),} \\
& {\left[\frac{\partial}{\partial \tau}+\left(\mu-\frac{\left(\mathcal{D}-\mathcal{D}_{o_{C J}}\right)}{\epsilon a}\right) \frac{\partial}{\partial \xi}\right] \mu=-\frac{l}{\epsilon r}(1+\mu) \text {. }}
\end{aligned}
$$

This is equation $(2.31)$.

\section{C.2. Solution to equation (2.55)}

The attention is focused on

$$
0<\mu_{f i} \ll 1 \quad \text { with } \quad \tilde{\xi}_{0 i}<0
$$

and one considers the positive solution $\tau_{t}>0$. Equation (2.55) can be put in the form

$$
\tilde{\tau}_{t}\left[1-\frac{\left|\tilde{\xi}_{0 i}\right|}{1+\mu_{f i}}\right]+\left|\tilde{\xi}_{0 i}\right| \frac{\mu_{f i}}{\left(1+\mu_{f i}\right)}=\left(1+\tilde{\tau}_{t}\right) \ln \left(1+\tilde{\tau}_{t}\right) .
$$

A graphical representation of the solution is plotted in Figure 14 showing that $\tilde{\tau}_{t} \ll 1$ if $0<\mu_{f i} \ll 1$. To leading order, a Taylor expansion in powers of $\tilde{\tau}_{t}$ then leads to

$$
\tau_{t}\left(1-\left|\tilde{\xi}_{0 i}\right|\right)+\left|\tilde{\xi}_{0 i}\right| \mu_{f i} \approx \tilde{\tau}_{t} \quad \Rightarrow \quad \tilde{\tau}_{t} \approx \mu_{f i}
$$


which is also right for $\tilde{\xi}_{0 i}=-1$ that is, according to the definition $(2.36)$, for $\left(r_{f i}-r_{0 i}\right)=$ $\epsilon r_{0 i}$ which is valid in the self-similar solution of the CJ detonation (2.26). In this particular case, equation (C 5) yields

$$
\left(1+\tilde{\tau}_{t}\right) \frac{\mu_{f i}}{\left(1+\mu_{f i}\right)}=\left(1+\tilde{\tau}_{t}\right) \ln \left(1+\tilde{\tau}_{t}\right) \quad \Rightarrow \quad \tilde{\tau}_{t}=\mathrm{e}^{\mu_{f i} /\left(1+\mu_{f i}\right)}-1 \approx \mu_{f i} .
$$

\section{C.3. Simplified expression for the external flow}

Discarding the integral term $Y(\tau) / b=O\left(y_{i} / b\right)$, the timescale of the external flow (4.15) is larger than the response of the inner structure of the detonation by a factor $\tilde{r}_{f i}$. According to (2.56), the time $\tau_{t}$, solution to the equation $\mu_{f}^{e x t}\left(\tau_{t}\right)=0$, is close to $\mu_{f i}^{e x t} \tilde{r}_{f i}$ if $\mu_{f i}^{e x t}$ is sufficiently smaller than unity,

$$
\mu_{f i}^{e x t} \ll 1 \Rightarrow \tau_{t} \approx \mu_{f i}^{e x t} \tilde{r}_{f i} \quad \forall \tilde{\xi}_{0 i} .
$$

Consider a large radius $\tilde{r}_{f i}$ and an initial propagation velocity close to the CJ velocity as in (2.41), for which

$$
\tilde{r}_{f i} \gg 1, \quad \mu_{f i}^{e x t} \ll 1, \quad \tilde{r}_{f i} \mu_{f i}^{e x t} \gg 1,
$$

so that the time $\tau_{t} \approx \tilde{r}_{f i} \mu_{f i}^{e x t}$ is larger than the characteristic time of the linear response of the inner structure which is of order unity, but $\tilde{\tau}_{t} \equiv \tau_{t} / \tilde{r}_{f i} \approx \mu_{f i}^{e x t} \ll 1$ is small. For a lapse of time $\tau$ of the same order as $\tau_{t}$, using (4.12),

$$
\tau=O\left(\tau_{t}\right), \quad \tilde{r}_{f}(\tau)=\tilde{r}_{f i}\left(1+\tau / \tilde{r}_{f i}\right) \approx \tilde{r}_{f i},
$$

the instantaneous reduced curvature $1 / \tilde{r}_{f}$ does not vary much and can be considered as a constant in (4.10). Under the condition (C 9), the thickness of the boundary layer (2.58) $\Delta|\tilde{\xi}| \approx \mu_{f i}^{e x t}, \Delta|\xi|=O\left(\mu_{f i}^{e x t} \tilde{r}_{f i}\right)$, is larger than the thickness of the inner structure which is of order unity for $\mathrm{e}^{-y}=O(1)$, so that the outer flow of an overdriven detonation reduces to $(2.59)$,

$$
\mu^{e x t}(\xi, \tau) \approx \frac{1}{\tilde{r}_{f i}}\left[\frac{\xi}{-\tilde{\xi}_{0 i}}+\left(\tau_{t}-\tau\right)\right], \quad \mu_{f}^{e x t}(\tau) \approx \frac{\left(\tau_{t}-\tau\right)}{\tilde{r}_{f i}},
$$

with a length-scale larger than the detonation thickness by a factor $\tilde{r}_{f i}\left|\tilde{\xi}_{0 i}\right|$ and a time scale larger than the response time of the inner structure by a factor $\tilde{r}_{f i} \gg 1$.

\section{C.4. Motion of the sonic point}

In overdriven regimes, the position of the sonic point $\xi=\xi_{s}(\tau)$ is related to the evolution of the detonation velocity $y(\tau) / b$. Introducing the relation $\mu^{e x t}\left(\xi_{s}(\tau), \tau\right)=y(\tau) / b$ into(4.15) and using (4.17) yields

$$
\begin{aligned}
& 1+\frac{y(\tau)}{b}=\frac{\xi_{s}(\tau)}{\tilde{r}_{f}(\tau)\left[\left(-\tilde{\xi}_{0 i}\right) /\left(1+\mu_{f i}^{e x t}\right)+\ln \left(\tilde{r}_{f} / \tilde{r}_{f i}\right)\right]}+\left(1+\mu_{f}^{e x t}(\tau)\right) \\
& \left.\left[1+\frac{y(\tau)}{b}\right]\left[\tilde{r}_{f}(\tau)\right) \frac{\left(-\tilde{\xi}_{0 i}\right)}{\left(1+\mu_{f i}^{e x t}\right)}+\tilde{r}_{f} \ln \left(\tilde{r}_{f} / \tilde{r}_{f i}\right)\right]=\xi_{s}(\tau)+\tau[1+Y(\tau)]+\left(-\tilde{\xi}_{0 i}\right) \tilde{r}_{f i} .
\end{aligned}
$$

According to (4.12), the relation $\tilde{r}_{f}(\tau)=\tau+\tilde{r}_{f i}$ leads to

$$
\xi_{s}(\tau)=\left\{\begin{array}{l}
\frac{y(\tau)}{b}\left[\left(\tau+\tilde{r}_{f i}\right) \frac{\left(-\tilde{\xi}_{0 i}\right)}{\left(1+\mu_{f i t}^{e x t}\right)}+\tilde{r}_{f} \ln \left(\tilde{r}_{f} / \tilde{r}_{f i}\right)\right] \\
\tau \frac{\left(-\tilde{\xi}_{0 i}\right)}{\left(1+\mu_{f i}^{e x t}\right)}-\tau-\frac{1}{b} \int_{0}^{\tau} y\left(\tau^{\prime}\right) \mathrm{d} \tau^{\prime}+\tilde{r}_{f} \ln \left(\tilde{r}_{f} / \tilde{r}_{f i}\right) \\
\tilde{r}_{f i} \frac{\left(-\tilde{\xi}_{0 i}\right)}{\left(1+\mu_{f i}^{e x t}\right)}-\left(-\tilde{\xi}_{0 i}\right) \tilde{r}_{f i}
\end{array}\right.
$$




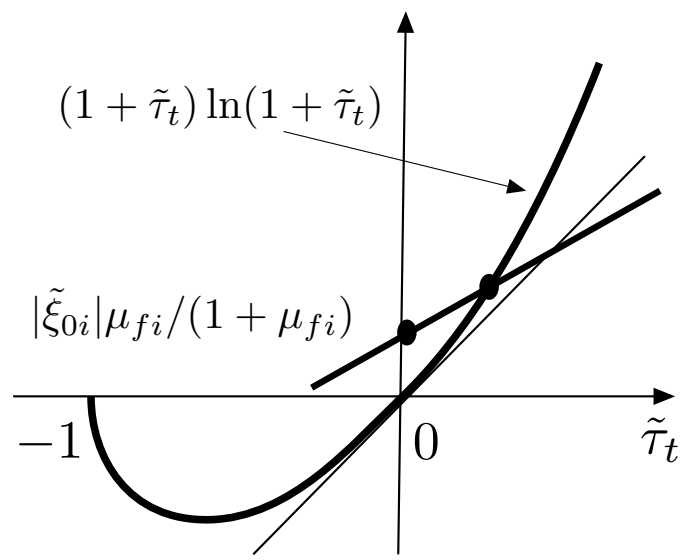

FiguRE 14. Graphical solution of (C 5) for $0<\mu_{f i} \ll 1$ and $\tilde{\xi}_{0 i}<0$. The thick straight line is the left-hand side of (C 5) plotted for $\left|\tilde{\xi}_{0 i}\right|<1+\mu_{f i}$

Using $\mathrm{d}\left[\tilde{r}_{f} \ln \left(\tilde{r}_{f} / \tilde{r}_{f i}\right)\right] / \mathrm{d} \tau=\ln \left(\tilde{r}_{f} / \tilde{r}_{f i}\right)+1$, derivation of (C 13) yields

$$
\frac{\mathrm{d} \xi_{s}}{\mathrm{~d} \tau}=\left\{\begin{array}{l}
\frac{1}{b} \frac{\mathrm{d} y}{\mathrm{~d} \tau}\left[\left(\tau+\tilde{r}_{f i}\right) \frac{\left(-\tilde{\xi}_{0 i}\right)}{\left(1+\mu_{f i}^{e x t}\right)}+\tilde{r}_{f} \ln \left(\tilde{r}_{f} / \tilde{r}_{f i}\right)\right] \\
\frac{y(\tau)}{b}\left[\frac{\left(-\tilde{\xi}_{0 i}\right)}{\left(1+\mu_{f i}^{e x t}\right)}+\ln \left(\tilde{r}_{f} / \tilde{r}_{f i}\right)+1\right] \\
\frac{\left(-\tilde{\xi}_{0 i}\right)}{\left(1+\mu_{f i}^{e x t}\right)}-1-\frac{y}{b}+\ln \left(\tilde{r}_{f} / \tilde{r}_{f i}\right)+1
\end{array}\right.
$$

to give

$$
\frac{\mathrm{d} \xi_{s}}{\mathrm{~d} \tau}=\left\{\begin{array}{l}
\frac{1}{b} \frac{\mathrm{d} y}{\mathrm{~d} \tau} \tilde{r}_{f}\left[\frac{\left(-\tilde{\xi}_{0 i}\right)}{\left(1+\mu_{f i}^{e x t}\right)}+\ln \left(\tilde{r}_{f} / \tilde{r}_{f i}\right)\right] \\
\frac{y(\tau)}{b}\left[\frac{\left(-\tilde{\xi}_{0 i}\right)}{\left(1+\mu_{f i}^{e x t}\right)}+\ln \left(\tilde{r}_{f} / \tilde{r}_{f i}\right)\right] \\
\frac{\left(-\tilde{\xi}_{0}\right)}{\left(1+\mu_{f i}^{e x t}\right)}+\ln \left(\tilde{r}_{f} / \tilde{r}_{f i}\right)
\end{array}\right.
$$

yielding

$$
\frac{1}{\left(-\tilde{\xi}_{0 i}\right) /\left(1+\mu_{f i}^{e x t}\right)+\ln \left(\tilde{r}_{f} / \tilde{r}_{f i}\right)} \frac{\mathrm{d} \xi_{s}}{\mathrm{~d} \tau}=\tilde{r}_{f} \frac{1}{b} \frac{\mathrm{d} y}{\mathrm{~d} \tau}+\left[1+\frac{y(\tau)}{b}\right]
$$

The coefficient of $\mathrm{d} \xi_{s} / \mathrm{d} \tau$ on the left-hand side being positive, the sign of $\mathrm{d} \xi_{s} / \mathrm{d} \tau$ is the same as $\tilde{r}_{f} \mathrm{~d}[1+y(\tau) / b] / \mathrm{d} \tau+[1+y(\tau) / b]$ in the right-hand side.

\section{Appendix D. Relaxation of spherical shock waves in open space}

Introducing the reduced propagation velocity $\dot{\alpha}_{\tau}$ defined in $(2.30), 1+\dot{\alpha}_{\tau} \approx(\mathcal{D}-a) / \epsilon a$, the relaxation of an inert shock wave in spherical geometry corresponds to

$$
\mathcal{D} \geqslant a \quad \Leftrightarrow \quad \dot{\alpha}_{\tau} \geqslant-1, \quad(\mathcal{D}-a) / a \rightarrow 0^{+}:\left(\dot{\alpha}_{\tau}+1\right) \rightarrow 0^{+} .
$$

Therefore the end of the decay cannot be described by the approximation $\left|\dot{\alpha}_{\tau}\right| \ll 1$ used in (2.39). At the Neumann state $(\xi=0)$, the flow is subsonic with respect to the shock $\left(\mathcal{D}-u_{f}\right) \leqslant a$, and, according to the Rankine-Hugoniot condition for $(\mathcal{D}-a) / a \ll 1$, the boundary condition at the shock front reads $u_{f} \approx 2(\mathcal{D}-a)$ see (A 1)-(A 2) for $Q=0$, which, using $\mu+1 \equiv u / \epsilon a$ in (2.28), corresponds to $\mu_{f}=1+2 \dot{\alpha}_{\tau}$. Focusing the attention to an initial velocity $\mathcal{D}_{i}>a$ close to the sound speed, the function $\mathcal{D}(t)$ is decreasing 
monotonously from an initial value $\left(\mathcal{D}_{i}-a\right) / a \ll 1$ to $a$. This is an eigenvalue problem consisting in determining the decreasing function $\dot{\alpha}_{\tau}(\tilde{\tau}) \rightarrow-1\left(\mu_{f}(\tilde{\tau}) \rightarrow-1\right)$, solution of

$$
\frac{\partial \mu}{\partial \tilde{\tau}}+\left(\mu-\dot{\alpha}_{\tau}\right) \frac{\partial \mu}{\partial \tilde{\xi}}=-\frac{(1+\mu)}{\tilde{\tau}+1}
$$

satisfying the boundary condition

$$
\tilde{\xi}=0: \mu=\mu_{f}(\tilde{\tau}), \quad \mu_{f}=1+2 \dot{\alpha}_{\tau}
$$

for a given initial condition

$$
\tau=0: \quad \dot{\alpha}_{\tau}=\dot{\alpha}_{\tau i}, \quad \dot{\alpha}_{\tau i}>-1 \quad \Rightarrow \quad \mu_{f i}>-1 .
$$

The flow of the rarefaction wave decreases from its Neumann value at the shock to $u=0$ when the distance from the shock increases, so that the instantaneous flow field $\mu(\tilde{\xi}, \tilde{\tau})$ decreases from $1+2 \dot{\alpha}_{\tau}(\tilde{\tau})$ at $\tilde{\xi}=0$ to $\mu=-1$ at $\tilde{\xi}=\tilde{\xi}_{0}(\tilde{\tau})<0$ corresponding to the radius of the sphere of quiescent gas $(u=0)$. This point is a weak discontinuity propagating with the speed of the sound in the laboratory frame, according to (2.33),

$$
\tilde{\xi}=\tilde{\xi}_{0}(\tilde{\tau}): \mu=-1, \quad \mathrm{~d} \tilde{\xi}_{0} / \mathrm{d} \tilde{\tau}=-\left(1+\dot{\alpha}_{\tau}\right),
$$

since $\mathrm{d} \tilde{\xi}_{0} / \mathrm{d} \tilde{\tau}=\mathrm{d} \xi_{0} / \mathrm{d} \tau$. As before, equation (D 5 ) is automatically satisfied by the solution of (D 2) which reduces to $\partial \mu / \partial \tilde{\tau}-\left(1+\dot{\alpha}_{\tau}\right) \partial \mu / \partial \tilde{\xi}=0$ at $\mu=-1$. Notice that the scalar field $\left[\mu(\tilde{\xi}, \tilde{\tau})-\dot{\alpha}_{\tau}(\tilde{\tau})\right]$ decreases from a positive value $1+\dot{\alpha}_{\tau}$ at the shock $\tilde{\xi}=0$ to a negative value $-\left(1+\dot{\alpha}_{\tau}\right)$ at $\tilde{\xi}_{0}(\tilde{\tau})<0$ so that there should be a sonic point (relative to the shock) inside the rarefaction wave at the point where $\left(\mu-\dot{\alpha}_{\tau}\right)=0 \Leftrightarrow(\mathcal{D}-u)=a$.

Equation (D 2) has solutions in the same form as (2.43) and (2.45)-(2.47) are still valid since $\dot{\alpha}_{\tau}$ is eliminated from (2.44) when using $\partial \mu / \partial \tilde{\tau}=\dot{A}+\left(1+\dot{\alpha}_{\tau}\right) A B^{\prime}$ and $\left(\mu-\dot{\alpha}_{\tau}\right) \partial \mu / \partial \tilde{\xi}=\left(A B-1-\dot{\alpha}_{\tau}\right) A B^{\prime}$. Then, following the same development as in $(2.45)-$ (2.48), the solution to equation (D 2), $\mu(\tilde{\xi}, \tilde{\tau})$ and $\mu_{f}(\tilde{\tau})$, expressed in terms of $\tilde{\xi}_{0}(\tilde{\tau})$ and the initial values $\tilde{\xi}_{0 i}$ and $\mu_{f i}$, take the same form as (2.49)-(2.50), obtained without using the boundary condition at the front. Using the boundary condition (D 3) at the front and equation (D 5) in the form

$$
-\mathrm{d} \tilde{\xi}_{0} / \mathrm{d} \tilde{\tau}=\left(1+\dot{\alpha}_{\tau}\right)=\left[\mu_{f}(\tilde{\tau})+1\right] / 2
$$

the expression of $\mu_{f}(\tilde{\tau})$ in $(2.50)$ yields an ordinary differential equation for $\tilde{\xi}_{0}(\tilde{\tau})$

$$
\frac{1}{\tilde{\xi}_{0}(\tilde{\tau})} \frac{\mathrm{d} \tilde{\xi}_{0}(\tilde{\tau})}{\mathrm{d} \tilde{\tau}}=\frac{1}{2(1+\tilde{\tau})\left[\theta_{i}+\ln (1+\tilde{\tau})\right]} \quad \text { where } \quad \theta_{i} \equiv-\frac{\tilde{\xi}_{0 i}}{\left(1+\mu_{f i}\right)}>0
$$

This equation can be integrated by considering the integral

$$
I(\tau)=\int_{0}^{\tau} \frac{\mathrm{d} \tilde{\tau}^{\prime}}{\left(1+\tilde{\tau}^{\prime}\right)\left[\theta_{i}+\ln \left(1+\tilde{\tau}^{\prime}\right)\right]}=\ln \left[1+\frac{1}{\theta_{i}} \ln (1+\tilde{\tau})\right]
$$

obtained by the change of variable $Y=\ln \left(1+\tilde{\tau}^{\prime}\right)$ leading to

$$
\tilde{\xi}_{0}(\tilde{\tau}) / \tilde{\xi}_{0 i}=\sqrt{1+\frac{1}{\theta_{i}} \ln (1+\tilde{\tau})}, \quad \Rightarrow \quad \frac{\mathrm{d} \tilde{\xi}_{0}}{\mathrm{~d} \tilde{\tau}}=\frac{\tilde{\xi}_{0 i}}{2} \frac{1}{\theta_{i}(1+\tilde{\tau}) \sqrt{1+\frac{1}{\theta_{i}} \ln (1+\tilde{\tau})}}(\mathrm{D} 9)
$$

Putting together (D 6) and (D 9) provides us with the propagation velocity $\dot{\alpha}_{\tau}(\tilde{\tau})$

$$
1+\dot{\alpha}_{\tau}(\tilde{\tau})=\frac{-\tilde{\xi}_{0 i} / 2}{\theta_{i}(1+\tilde{\tau}) \sqrt{1+\frac{1}{\theta_{i}} \ln (1+\tilde{\tau})}}
$$


showing a relaxation toward the sound speed in the long time limit in the form

$$
\lim _{\tilde{\tau} \rightarrow \infty}\left[1+\dot{\alpha}_{\tau}(\tilde{\tau})\right]=\frac{\text { cst. }}{\tilde{\tau} \sqrt{\ln \tilde{\tau}}} \Rightarrow \lim _{t \rightarrow \infty} \frac{\mathcal{D}(t)-a}{a}=\frac{\text { cst. }}{t \sqrt{\ln t}} .
$$

\section{Appendix E. Steady-state approximation for large activation energy}

The quasi-steady approximation pointed out a curvature-induced quenching which is worth comparing with the unsteady trajectories. The steady-state approximation is not expected to be an accurate approximation in the direct initiation of spherical gaseous detonation, because the unsteady terms are of the same order of magnitude as the curvature term, see the discussion in Clavin \& Denet (2020). In this appendix the quasi-steady approximation of the inner structure is revisited in the limit of small heat release for large activation energy when the unsteady gradient of the burnt gas flow of overdriven detonations is taken into account. Then, when the unsteady term on the left-hand side of (4.21) is neglected, the problem reduces to

$$
\begin{aligned}
& \left(\mu-\frac{y}{b}\right) \frac{\partial \mu}{\partial \xi}-\left(\mu^{e x t}-\frac{y}{b}\right) \frac{\partial \mu^{e x t}}{\partial \xi}=\frac{1}{2} \mathrm{e}^{y(\tau)} \omega_{O_{C J}}\left(\xi \mathrm{e}^{y(\tau)}\right)-\frac{\hat{\mu}}{\tilde{r}_{f}}, \\
& \xi=0: \quad \mu=1+2 y(\tau) / b ; \quad \xi \leqslant-\mathrm{e}^{-y(\tau)}: \hat{\mu}=0 .
\end{aligned}
$$

Equations (E 2)-(E 2) correspond to a better quasi-steady approximation than in Clavin \& Denet (2020), because here, the unsteady effect of the external flow is retained, assuming a steady-state approximation only for the inner structure of the overdriven detonation. Equation (E 2) can be written

$$
\frac{\partial}{\partial \xi}\left[\left(\mu-\frac{y}{b}\right)^{2}-\left(\mu^{e x t}-\frac{y}{b}\right)^{2}\right]=\frac{\partial}{\partial \xi}\left[\mu_{O_{C J}}\left(\xi \mathrm{e}^{y}\right)\right]^{2}-2 \frac{\hat{\mu}}{\tilde{r}_{f}}
$$

and its integration from the end of the reaction zone where, according to (4.23) or (E 2) $\xi=-\mathrm{e}^{-y}: \mu=\mu^{e x t}(\hat{\mu}=0)$, leads to

$$
\left(\mu-\frac{y}{b}\right)^{2}-\left(\mu^{e x t}-\frac{y}{b}\right)^{2}=\mu_{o_{C J}}^{2}\left(\xi \mathrm{e}^{y}\right)-\frac{2}{\tilde{r}_{f}} \int_{-\mathrm{e}^{-y}}^{\xi} \hat{\mu}\left(\xi^{\prime}, \tau\right) \mathrm{d} \xi^{\prime} .
$$

Then, the boundary condition on the lead shock in (4.11) or (E 2$), \xi=0: \mu=1+2 y(\tau) / b$, $\mu^{e x t}=\mu_{f}^{e x t}(\tau), \mu_{o_{C J}}=1$, provides us with an implicit relation for $y(\tau)$ involving the solution $\hat{\mu}(\xi, \tau)$

$$
2\left(1+\mu_{f}^{e x t}\right) \frac{y}{b}=\left(\mu_{f}^{e x t}\right)^{2}-\frac{2}{\tilde{r}_{f}} \int_{-\mathrm{e}^{-y}}^{0} \hat{\mu}\left(\xi^{\prime}, \tau\right) \mathrm{d} \xi^{\prime},
$$

A closed equation relating $y(\tau)$ and $\mu_{f}^{e x t}(\tau)$ is obtained for a large activation energy $b \gg 1$, since $\hat{\mu}(\xi, \tau)$ can be replaced by $\mu_{o_{C J}}\left(\xi \mathrm{e}^{y}\right)$ in the integral term

$$
b \gg 1: \quad 2\left(1+\mu_{f}^{e x t}\right) \frac{y}{b} \approx\left(\mu_{f}^{e x t}\right)^{2}-\frac{2 \mathrm{e}^{-y}}{\tilde{r}_{f}} \int_{-1}^{0} \mu_{o_{C J}}(\xi) \mathrm{d} \xi,
$$

as shown now. Looking for a solution corresponding to $y$ of order unity and $\tilde{r}_{f}$ of order $b, r_{f i} / l=O(b / \epsilon)$, one is led to consider $\left(\mu_{f}^{e x t}\right)^{2}=O(1 / b)$ in (E 6$)$. This is consistent with the simplified expression (C11) of the rarefaction wave behind a overdriven detonation

$$
\mu_{f}^{e x t}(\tau)=\frac{\left(\tau_{t}-\tau\right)}{\tilde{r}_{f i}}=O(1 / \sqrt{b}), \quad \mu_{f i}^{e x t}=\frac{\tau_{t}}{\tilde{r}_{f i}}=O(1 / \sqrt{b}),
$$


leading to

$$
b \gg 1, \quad \tilde{r}_{f} \approx \tilde{r}_{f i}=O(b), \quad \tau=O\left(\tau_{t}\right), \quad \tau_{t}=O(\sqrt{b}) .
$$

Then, the zeroth-order $\hat{\mu}_{o}\left(\xi^{\prime}, \tau\right)$ of $\hat{\mu}\left(\xi^{\prime}, \tau\right)$ in the limit $b \gg 1$ can be used in the integral term on the right-hand side of (E 5). This zeroth-order solution $\hat{\mu}_{o}\left(\xi^{\prime}, \tau\right)$ is solution to the steady version of (4.21)-(4.23) when the terms smaller than unity are neglected,

$$
\hat{\mu}_{o} \frac{\partial \hat{\mu}_{o}}{\partial \xi}=\frac{1}{2} \mathrm{e}^{y} \omega_{o_{C J}}\left(\xi \mathrm{e}^{y}\right), \quad \xi=0: \hat{\mu}_{o}=1, \quad \xi \leqslant-\mathrm{e}^{-y}: \hat{\mu}_{o}=0,
$$

yielding, according to (4.6)-(4.7), $\hat{\mu}_{o}\left(\xi^{\prime}, \tau\right)=\mu_{o_{C J}}\left(\xi^{\prime} \mathrm{e}^{y(\tau)}\right)$. This leads to the transcendental equation for $y$ in (E 6) which, neglecting the term $\mu_{f}^{e x t}$ on the left-hand side in front of unity, can be written in the form

$$
b \gg 1: \quad y+\frac{\mathrm{e}^{-y}}{x}=\frac{1}{2} b\left[\mu_{f}^{e x t}(\tau)\right]^{2} \quad \text { where } \quad x \equiv \frac{\tilde{r}_{f}}{b} \frac{1}{\int_{-1}^{0} \mu_{O_{C J}}\left(\xi^{\prime}\right) \mathrm{d} \xi^{\prime}}=O(1),
$$

and $b\left[\mu_{f}^{e x t}(\tau)\right]^{2}=O(1)$, see $(\mathrm{C} 10)$ and $(\mathrm{E} 7)$, the integral $\int_{-1}^{0} \mu_{o_{C J}}\left(\xi^{\prime}\right) \mathrm{d} \xi^{\prime}$ being close to 1/2. Using (4.12) $\tilde{r}_{f} \approx \tau+\tilde{r}_{f i}$ and the simplified expression $\mu_{f}^{e x t}(\tau)$ in (E 7$)$ to eliminate $\tau$ in favor of $x$, equation (E 10) yields the leading order in the limit $b \gg 1$ of the quasisteady trajectories in the phase-space "velocity-radius" $y-x$. Using the substitutions $\sqrt{b / 2} \mu_{f}^{e x t} \rightarrow \sqrt{b / 2} \mu_{b} \equiv \mathrm{m}_{b}$ and $\int_{-1}^{0} \mu_{o_{C J}}\left(\xi^{\prime}\right) \mathrm{d} \xi^{\prime} \rightarrow 1+\int_{-1}^{0} \mu_{o_{C J}}\left(\xi^{\prime}\right) \mathrm{d} \xi^{\prime}$, equation (E 10) takes the same form as (7.1a) in Clavin \& Denet (2020) and the trajectories have a form similar to figure 1 ibid. with a definition of $x$ which differs by a factor of order unity, compare (4.20a-b) ibid. with (E 10). In particular, the critical radius $r_{f}^{*}$ at the turning point $(y=-1, x=\mathrm{e})$ of the C-shaped curve "CJ velocity - radius", obtained from (E 10) for $b\left[\mu_{f}^{e x t}(\tau)\right]^{2}=0$, is

$$
b \gg 1: \quad \frac{r_{f}^{*}}{l}=\frac{b}{\epsilon} \mathrm{e} \int_{-1}^{0} \mu_{o_{C J}}\left(\xi^{\prime}\right) \mathrm{d} \xi^{\prime}
$$

while it was $r_{f}^{*} / l=(b / \epsilon) \mathrm{e}\left[1+\int_{-1}^{0} \mu_{o_{C J}}\left(\xi^{\prime}\right) \mathrm{d} \xi^{\prime}\right]$ in (4.20c) of Clavin \& Denet (2020) where the gradient of the burnt-gas flow was ignored. In any case the critical radius corresponds to a non-dimensional radius $\tilde{r}_{f} / b$ of order unity. 


\section{REFERENCES}

Clavin, P. \& Denet, B. 2018 Decay of plane detonation waves to the self-propagating Chapman-Jouguet regime. J. Fluid. Mech. 845, 170-202.

Clavin, P. \& Denet, B. 2020 Analytical study of the direct initiation of gaseous detonations for small heat release. J. Fluid. Mech. 897, A30.

Clavin, P. \& Searby, G. 2016 Combustion waves and fronts in flows. Cambridge University Press.

Clavin, P. \& Williams, F.A. 2002 Dynamics of planar gaseous detonations near ChapmanJouguet conditions for small heat release. Combustion Theory and Modelling 6, 127-129.

Eckett, C.A., Quirk, J.J. \& Shepherd, J.E. 2000 The role of unsteadiness in direct initiation of gaseous detonations. J. Fluid. Mech. 421, 147-183.

HE, L. 1996 Theoretical determination of the critical conditions for the direct initiation of detonations in hydrogen-oxygen mixtures. Combust. Flame 104, 401-418.

He, L. \& Clavin, P. 1994 On the direct initiation of gaseous detonations by an energy source. Journal of Fluid Mechanics 277, 227-248.

Liñan, A., Kurdyumov, V. \& Sanchez, A.L. 2012 Initiation of reactive blast waves by external energy source. Comptes Rendus Mécanique 340, 829-844.

NG, H. \& LEE, J.H.S. 2003 Direct initiation of detonation with a multi-step reaction scheme. Journal of Fluid Mechanics 476, 179-211.

Sanchez, A.L. \& Williams, F.A. 2014 Recent advances in understanding of flammability characteristics of hydrogen. Progress in Energy and Combustion Science 41, 1-55.

Sedov, L.I. 1946 Propagation of strong blast waves. Prikl. Mat. Meckh. 10, 241-250.

TAYLOR, G. I. 1950a The dynamics of combustion products behind plane and spherical detonation fronts. Proc. R. Soc. London A 200, 235-247.

TAYLOR, G. I. 1950b The formation of a blast wave by a very intense explosion. Proc. R. Soc. London A 201, 159-174.

ZeLDOVICH, YA. B. 1942 On the distribution of pressure and velocity in products of detonation blasts, in particular for spherical propagating detonation waves. Zhur. Eksp. Teor. Fiz. 12, 389-406. 